\title{
Characterization of the psycho-neuro-immunological state in patients with coronary artery disease
}

\author{
Ph.D. Thesis
}

Gyöngyi Serfőző MSc

Szeged

2016 


\section{Characterization of the psycho-neuro- immunological state in patients with coronary artery disease}

Ph.D. Thesis

Gyöngyi Serfőző MSc

Doctoral school:

Multidisciplinary Doctoral School

Supervisor:

Margit Keresztes M.D. Ph.D.

Department of Biochemistry

Faculty of Medicine

University of Szeged

Szeged

2016 


\section{List of papers related to the subject of the thesis}

I. Margit Keresztes, Tamás Horváth, Imre Ocsovszki, Imre Földesi, Gyöngyi

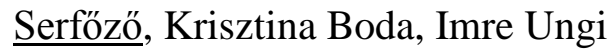

ACTH- and cortisol-associated neutrophil modulation in coronary artery disease patients undergoing stent implantation

PLoS One (Published: Aug 14, 2013)

DOI: 10.1371/journal.pone.0071902

IF. 3.534

II. Gyöngyi Serfőző, Tamás Horváth, Imre Földesi, Beatrix Rafael, Tamás Forster, Imre Ungi, Roland von Känel, Margit Keresztes

The monocyte-to-lymphocyte ratio correlates with psycho-neuroinflammatory factors in patients with stable coronary artery disease Neuroimmunomodulation (Published online: March 1, 2016)

DOI: $10.1159 / 000443835$

IF: $1.882(2013 / 14)$ 


\section{Table of Contents}

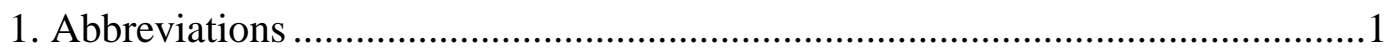

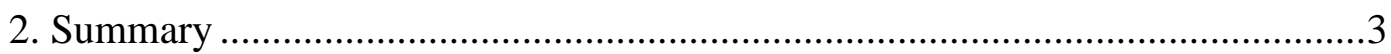

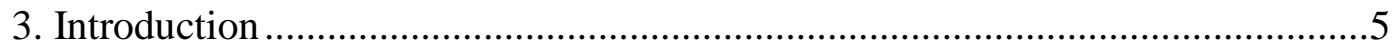

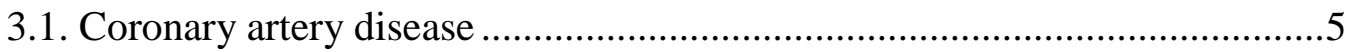

3.2. The process of atherosclerosis - main points ...........................................

3.3. Roles of neutrophils and monocytes in coronary artery disease .................11

3.4. Psychosocial stress and coronary artery disease (psycho-neuro-

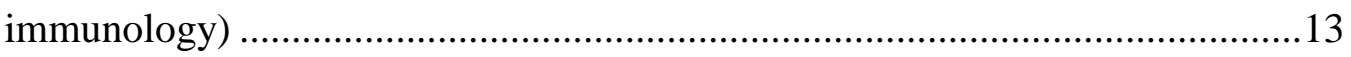

3.5. Blood plasma inflammatory factors in CAD ...........................................15

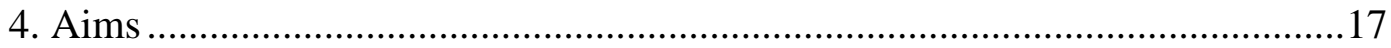

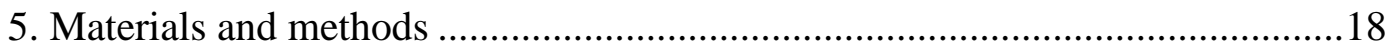

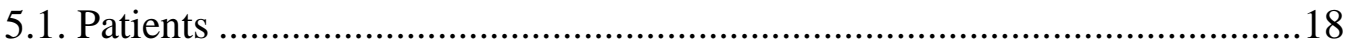

5.2. Blood sampling and laboratory methods ...............................................20

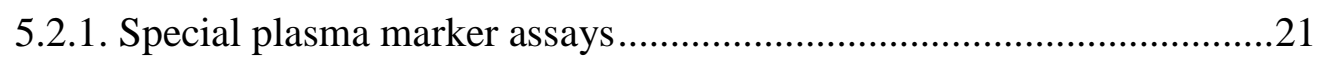

5.2.1.1. Neuroendocrine (stress) markers.............................................. 21

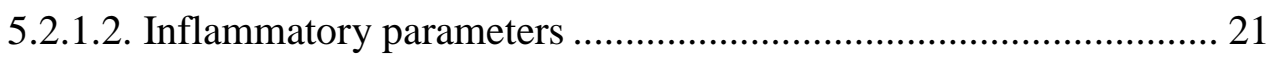

5.2.2. Determination of surface granulocyte activation markers (study I.) ....22

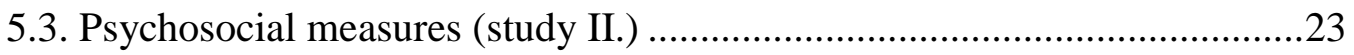

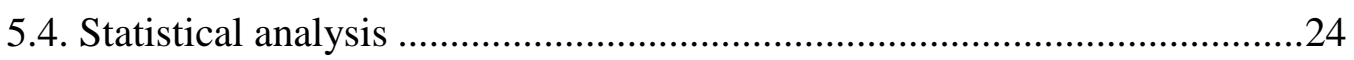

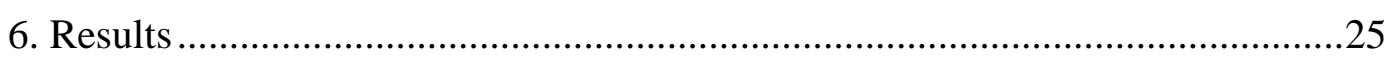

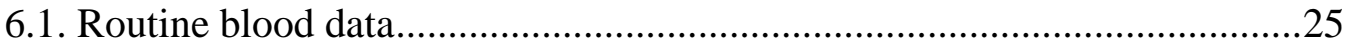

6.2. Special laboratory assays from blood samples ........................................27

6.2.1. Neuroendocrine markers: cortisol, ACTH, chromogranin A ...............27

6.2.2. Special inflammatory markers in plasma: lactoferrin, IL-6, LL-37 .....28

6.2.3. Surface activation markers of granulocytes: L-selectin, CD15,

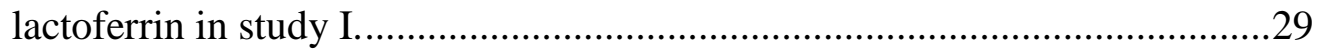

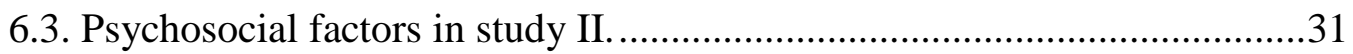

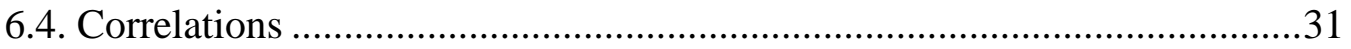


6.4.1. Correlations between stress hormones (cortisol, ACTH) and selected markers: percentage of lactoferrin-bearing neutrophils, plasma lactoferrin and IL-6 in study I.

6.4.2. Correlations between the MLR and psychosocial factors, neuroendocrine and inflammatory parameters in study II.

6.4.3. Correlations amongst psychosocial factors, neuroendocrine and inflammatory factors in study II.

7. Discussion .36

7.1. Stress and inflammatory parameters in CAD patients undergoing stenting 37 7.2. ACTH- and cortisol-associated neutrophil modulation in CAD patients undergoing stenting 39

7.3. The monocyte-to-lymphocyte ratio: a PNI correlate in stable CAD .

7.4. Principal findings .42

7.5. Limitations. .43

8. Conclusion .44

9. Acknowledgements .45

10. References .46

11. Annex .61 


\section{Abbreviations}

\begin{tabular}{|c|c|}
\hline ACS & acute coronary syndrome \\
\hline ACTH & adrenocorticotropic hormone \\
\hline ANOVA & analysis of variance \\
\hline BDI & Beck Depression Inventory \\
\hline BMI & body mass index \\
\hline $\mathrm{BSCI}$ & Rahe's Brief Stress and Coping Inventory \\
\hline CAD & coronary artery disease \\
\hline $\mathrm{CgA}$ & chromogranin $\mathrm{A}$ \\
\hline CK & creatine kinase \\
\hline $\mathrm{CRH}$ & corticotropin-releasing hormone \\
\hline CRP & C-reactive protein \\
\hline $\mathrm{CV}$ & coefficient of variation \\
\hline ECG & electrocardiography / electrocardiogram \\
\hline ELISA & enzyme-linked immunosorbent assay \\
\hline FITC & fluorescein isothiocyanate \\
\hline HDL & high density lipoprotein \\
\hline HPA & hypothalamic-pituitary-adrenocortical \\
\hline IL-6 & interleukin-6 \\
\hline LDL & low density lipoprotein \\
\hline MFI & mean fluorescence intensity \\
\hline MI & myocardial infarction \\
\hline MLR & monocyte-to-lymphocyte ratio \\
\hline NYHA & New York Heart Association \\
\hline n.a. & not available \\
\hline n.s. & not significant \\
\hline PCI & percutaneous coronary intervention \\
\hline
\end{tabular}




$\begin{array}{ll}\text { PNI } & \text { psycho-neuroendocrino-inflammatory / } \\ & \text { psycho-neuro-immune / psycho-neuro-immunology / } \\ & \text { psycho-neuro-inflammatory } \\ \text { post-PCI } & \text { directly after percutaneous coronary intervention } \\ \text { pre-PCI } & \text { directly before percutaneous coronary intervention } \\ \text { r } & \text { correlation coefficient } \\ \text { SAM / SAS } & \text { sympathetic-adrenomedullary system } \\ \text { SAP } & \text { stable angina pectoris } \\ \text { SEM } & \text { standard error of the mean } \\ \text { SD } & \text { standard deviation } \\ \text { SNS } & \text { sympathetic nervous system } \\ \text { TBS } & \text { tris-buffered saline } \\ \text { TMB } & 3,3^{\prime}, 5,5 \text { '-tetramethylbenzidine } \\ \text { TNF- } \alpha & \text { tumour necrosis factor-alpha } \\ \text { 1d-PCI } & \text { on the following day of PCI }\end{array}$




\section{Summary}

Background. Psychosocial stress and depression are becoming widely recognized as major risk factors for coronary artery disease (CAD). Although both neutrophil granulocytes and monocytes are known as principal contributors to atherosclerosis, their associations with psycho-neuroendocrino-inflammatory (PNI) factors have not been fully revealed in CAD patients to date.

Objectives. Our aims were to examine cortisol- and ACTH-based alterations in granulocyte activation in stable and acute CAD patients undergoing percutaneous coronary intervention (PCI) and to study the correlations between the monocyte-to-lymphocyte ratio (MLR) and key PNI markers in stable CAD patients.

Methods. In study I., blood samples of 21 stable angina pectoris (SAP) and 20 acute coronary syndrome (ACS) patients were collected directly before (pre-PCI), after (post-PCI) and on the following day of PCI (1d-PCI). Granulocyte surface L-selectin, CD15 and (neutrophil-specific) lactoferrin were assayed by flow cytometry. Plasma cortisol, ACTH, lactoferrin and IL-6 were also analysed. In study II., 23 stable CAD patients participated who completed the Beck Depression Inventory (BDI) and Rahe's Brief Stress and Coping Inventory. A white blood cell differential and determinations of cortisol, chromogranin A (CgA), LL-37, interleukin-6 (IL6), and C-reactive protein (CRP) levels were carried out in plasma.

Results. In study I., pre- and post-PCI percentages of lactoferrin-carrying neutrophils were high and decreased markedly next day in both patient groups; similarly, 1d-PCI plasma lactoferrin values were about half of the post-PCI values (all: $\mathrm{p} \leq 0.0001$ ). Post-PCI ACTH reduced significantly one day after stenting, especially in ACS group (SAP: $p<0.01$, ACS: $p \leq 0.0001$ ). In ACS patients, the increased pre-PCI cortisol level decreased substantially the next day after stenting $(\mathrm{p}<0.01)$; in pre-PCI samples of this group, cortisol correlated with plasma lactoferrin $(\mathrm{r} \sim 0.5, \mathrm{p}<0.05)$. In 1d-PCI samples of both groups, ACTH associated negatively with the ratio of lactoferrin-bearing neutrophils (SAP: $r=-0.601, p<0.005$; ACS: $r=-0.541, p<0.05$ ) and with plasma lactoferrin (SAP: $r=-0.435, p<0.05 ;$ ACS: $r=-0.609, p<0.005$ ).

In study II., elevated monocyte fraction, MLR and plasma CgA levels characterized the stable CAD patients. Social support score was low and BDI scores of seven patients were increased. In the multivariate-adjusted analysis, the MLR correlated with depressive symptom severity 
$(\mathrm{r}=0.624, \mathrm{p}<0.01)$, plasma CgA $(\mathrm{r}=0.660, \mathrm{p}<0.01)$, LL-37 ( $\mathrm{r}=0.643, \mathrm{p}<0.01)$, IL-6 ( $\mathrm{r}=0.532$, $\mathrm{p}<0.05)$, and CRP values $(\mathrm{r}=0.470, \mathrm{p}<0.05)$. Higher BDI scores associated with higher concentrations of $\mathrm{CgA}(\mathrm{r}=0.618, \mathrm{p}<0.01)$, and $\mathrm{CgA}$ level correlated inversely with social support score $(\mathrm{r}=-0.511, \mathrm{p}<0.05)$.

Conclusions. In stable and acute CAD patients, both pre- and post-PCI states could be associated with increased ratio of activated/degranulated neutrophils shown by the elevated lactoferrin parameters, which declined the next day of PCI indicating a substantial decrease in neutrophil activation. The 1d-PCI lactoferrin values were associated negatively with plasma ACTH in both CAD groups. In the ACS group, before stenting, a correlation of cortisol with plasma lactoferrin was found, that could reflect an association of cortisol with neutrofil activation in extremely stressed CAD patients. We suggest that plasma lactoferrin might be considered as a 'neutrophil activity sensor' in CAD.

In stable CAD patients, increased MLR may be associated with depressive symptoms, with increased neuroendocrino-sympathetic activity and with inflammatory factors that are probably involved in the initiation and development of atherosclerosis. The elevated neuroendocrinosympathetic activity correlated with low social support and with depressive symptom severity. The MLR could serve as a simple, inexpensive, broadly available PNI correlate marker in stable CAD.

\section{Keywords:}

$\mathrm{ACTH}$, acute coronary syndrome, atherosclerosis, coronary artery disease, cortisol, depressive symptoms, inflammation/inflammatory factors, lactoferrin, monocyte-to-lymphocyte ratio, neutrophil activation, psychological stress, psycho-neuro-immunology, social support, stable coronary artery disease, stenting 


\section{Introduction}

\subsection{Coronary artery disease}

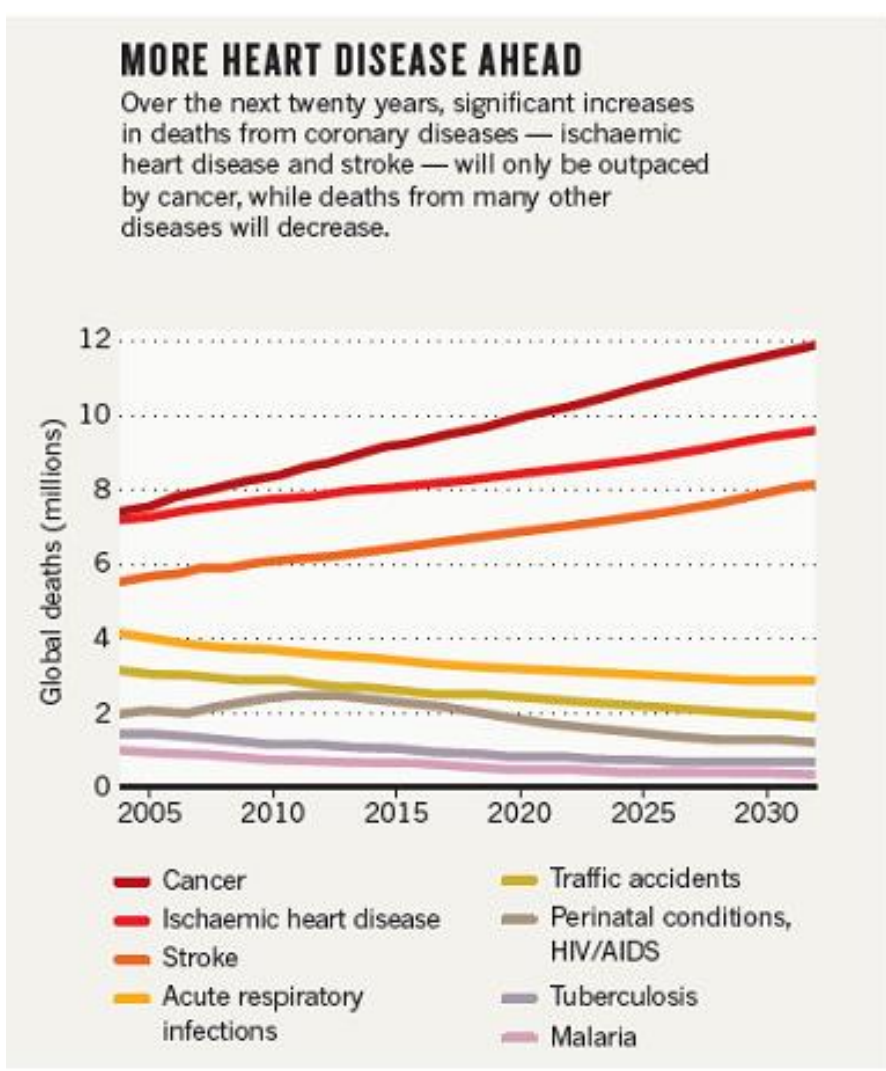

It is widely known, that coronary artery disease (CAD) is among the three leading causes of death in most countries worldwide [1-3]. Although its mortality rate reduced since the latter part of the $20^{\text {th }}$ century - mainly due to the advancement in both prevention and treatment (e.g. by introduction of percutaneous coronary intervention /PCI/) -, it is expected to remain the number 2 killer in 2030 worldwide, closely after cancer (Fig. 1) [4-7].

Figure 1. Cardiovascular disease (CVD) remains the grim reaper's primary calling card, but people can take steps to keep the world's number one killer at bay.

(Cannon B (2013) Cardiovascular disease: Biochemistry to behaviour. Nature 493:S2-3. doi:10.1038/493S2a.)

Traditionally, the main risk factors of CAD are partly life style factors: imbalanced diet, physical inactivity - and as a consequence of them: metabolic syndrome with abdominal obesity, dyslipoproteinemia, hypertension and insulin resistance (type 2 diabetes mellitus) and in addition, cigarette smoking; other risk factors include age, gender and genetic factors. The recognition of novel, psychological risk factors, like psychosocial stress and depression, is also emerging in $\mathrm{CAD}$; these conditions have gained much interest as potential key players in coronary atherosclerosis $[1,3,8]$.

Basically, CAD occurs as a consequence of atherosclerosis of the coronary arteries, which leads to limited blood flow and results in an imbalance between myocardial oxygen 
demand and supply. This hypoxia is responsible for angina pectoris symptoms, which may vary, but are mainly squeezing chest pain and/or referred pain that may radiate typically into the left arm, shoulder, back neck or jaw [9]. CAD can be classified to stable angina and acute coronary syndromes, the latter one may be further divided into three subgroups: unstable angina, non-ST elevation myocardial infarction (NSTEMI) and ST elevation myocardial infarction (STEMI) (Fig. 2) [10].

In stable angina, pain generally occurs predictably with exertional activity and resolves with rest or sublingual administration of nitroglycerin [9]. (Only the most serious stable cases are characterized by symptoms at rest). Unstable angina, a form of acute coronary syndromes,

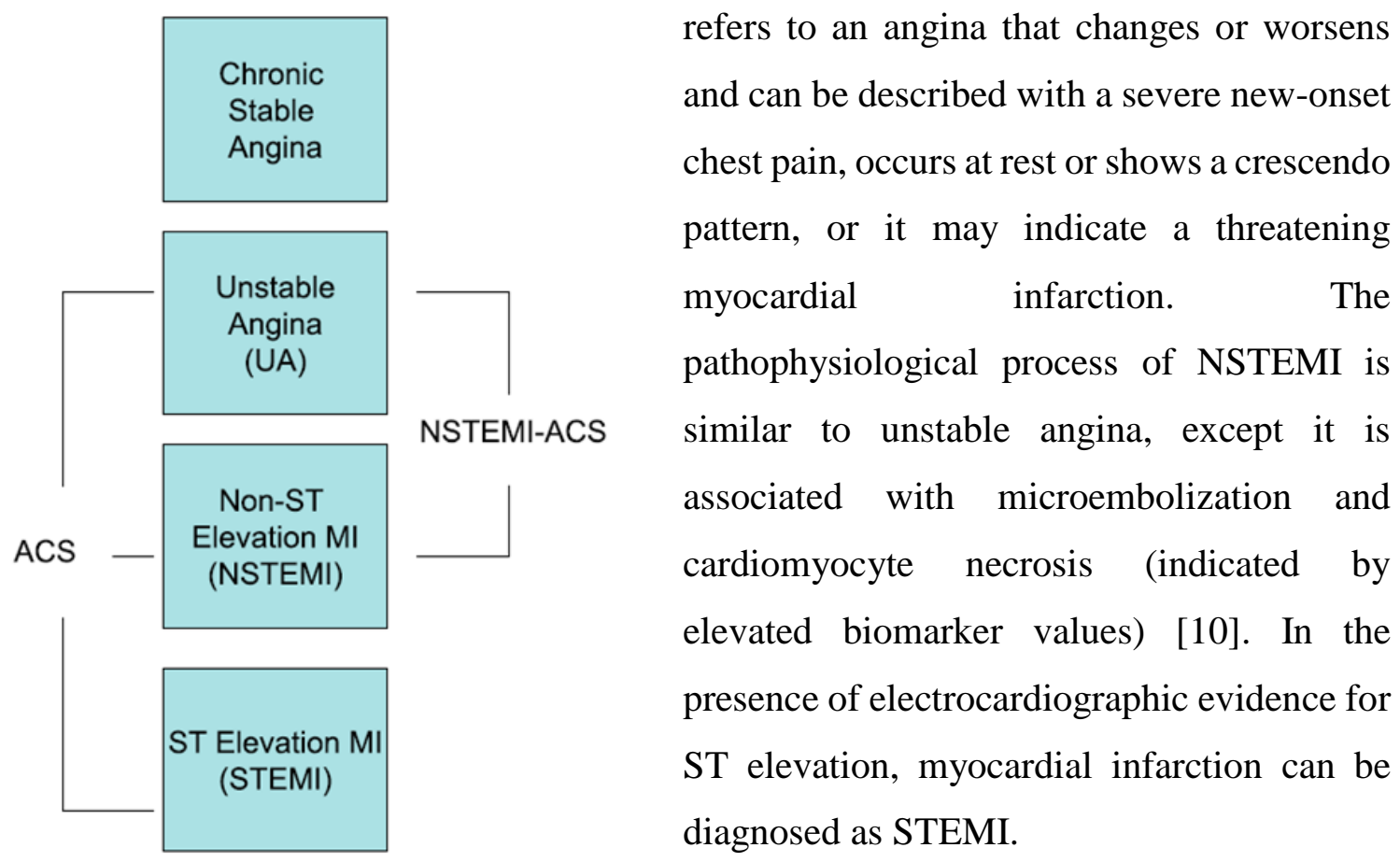

Figure 2. Spectrum of coronary artery disease. ACS = acute coronary syndrome.

(Braunwald E (2012) Unstable angina and non-ST elevation myocardial infarction. Am J Respir Crit Care Med 185:924-932. doi:10.1164/rccm.201109-1745CI.)

Myocardial infarction (MI) is classically defined as myocardial cell death owing to extended ischemia and caused predominantly by fibrous cap rupture and thrombus formation (MI type 1, 70\% of acute myocardial infarctions) [11]. 
Beside assessing the pattern of angina in symptomatic CAD patients, the main tools of differential diagnosis include electrocardiography (ECG), echocardiography, coronary angiography and biomarker testing. Recording an ECG and performing an echocardiogram at rest are routine diagnostic tests for all (suspected) CAD patients. Momentarily asymptomatic stable patients may perform an exercise stress test on a treadmill or a stationary cycle ergometer to get a 12-lead exercise ECG [12]. Coronary angiography (Fig. 3) is a minimally invasive

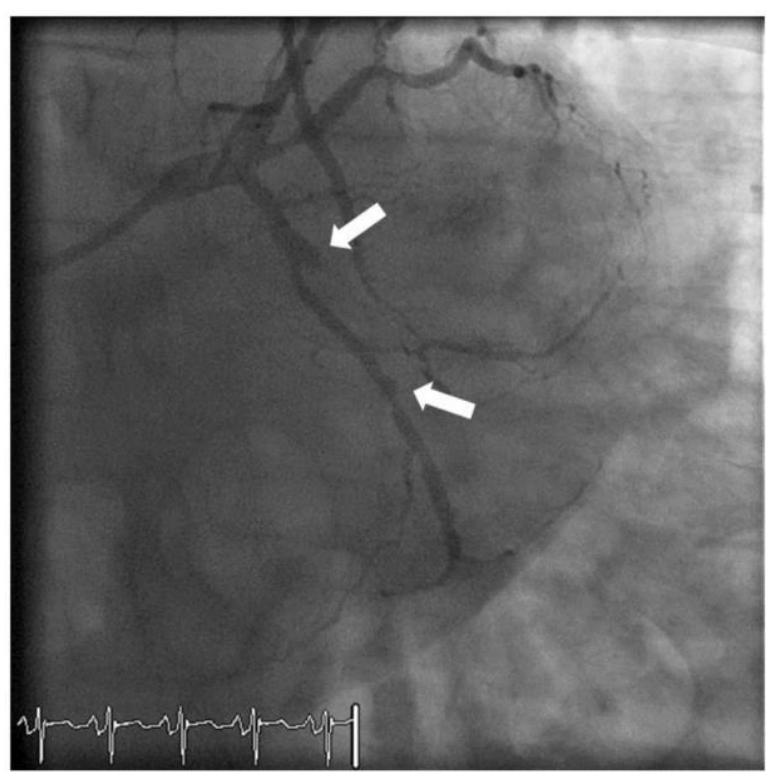
technic to assess coronary circulation, to identify the nature of the coronary lesion and to check whether the patient is suitable for percutaneous coronary intervention (PCI) or coronary artery bypass graft (CABG) surgery. Coronary angiography is performed with a tube-like catheter, which is led to the trunk of one of the coronary arteries and when in place, a radiocontrast dye is injected into the coronary blood to make the blood flow visible on the $\mathrm{X}$ ray motion picture.

Figure 3. Coronary angiogram demonstrating occluded arteries.

(Gladding PA, Cave A, Zareian M, Smith K, Hussan J, et al. (2013) Open access integrated therapeutic and diagnostic platforms for personalized cardiovascular medicine. J Pers Med 3:203-237. doi:10.3390/jpm3030203.)

\subsection{The process of atherosclerosis - main points}

Atherosclerosis is a multifocal, chronic inflammatory disease of the medium-sized and large arteries and it is the main underlying cause of coronary artery disease. According to autopsy studies, initial steps in the pathogenesis of atherosclerosis starts at young age and develops silently over decades before it leads to serious cardio- and cerebrovascular consequences [13]. Atherosclerosis alone remains rarely fatal; it is the superimposed thrombosis on the surface of an eroded or ruptured atherosclerotic plaque, that generally triggers the well-known life-threatening cardiovascular events such as acute coronary syndromes or stroke. Key players in the development of atherosclerosis are endothelial cells, leukocytes, and intimal smooth muscle cells (Fig. 4). 
On the very onset of this disease, endothelial dysfunction develops due to certain atherogenic factors (e.g. oxidative stress caused by smoking); then smaller plasma molecules and lipoprotein particles get through the leaky and defective endothelium into the subendothelial space. There, they get bound to proteoglycans in the basement membrane, accumulate, undergo an oxidative modification and become proinflammatory. Specifically, the oxidized LDL is the most significant proatherogenic contributor to the initiation of atherosclerosis. The mechanisms responsible for its formation from LDL is not fully known, although one of the possible triggers could be myeloperoxidase, which is most abundantly derived from neutrophil granulocytes [14,15].

As a result of proinflammatory stimuli, endothelium becomes activated, upregulates the expression of adhesion receptors - e.g. E-selectin, P-selectin and vascular cell adhesion molecule-1 (VCAM-1), and recruits primarily monocytes and T-cells - according to the traditional view. Cell surface upregulation of additional adhesion receptors (e.g. intercellular adhesion molecule-1/ICAM-1/), contribute to the further adhesion of blood-borne cells to the atherosclerotic lesion [16]. After transendothelial migration into the intima, monocytes differentiate into macrophages, phagocyte the proatherogenic lipoproteins via scavenger receptors and become fully lipid-loaded foam cells containing a huge amount of cholesteryl ester. However, a reverse cholesterol transport through the plasma membrane to extracellular HDL and to the liver is also possible under certain conditions [15,17,18].

The scavenger receptors are not down-regulated after cellular cholesterol accumulation, therefore, the endless supply of proinflammatory lipoproteins leads to the overloading and consequently, to the death of these leukocytes. Mass apoptosis of macrophages contributes to the formation of soft lipid-rich core in the lesion called the fatty streak, which is still asymptomatic, although activation and death of macrophages results in the release of a great variety of inflammatory, chemotactic and growth factors (IL-1 $\beta$, IL-6, IL-12, TNF- $\alpha$ ) [19].

In further steps of the progression of atherosclerosis, intimal smooth muscle cells mediate a fibroproliferative response to heal the inflamed and injured artery that leads to plaque formation. Although, when a prolonged inflammation persists, this reparative response could backfire and beside providing stability to plaques against rupture and thrombosis, contributes to the narrowing of the lumen and leads to reduced blood flow and ischemia [20,21]. 


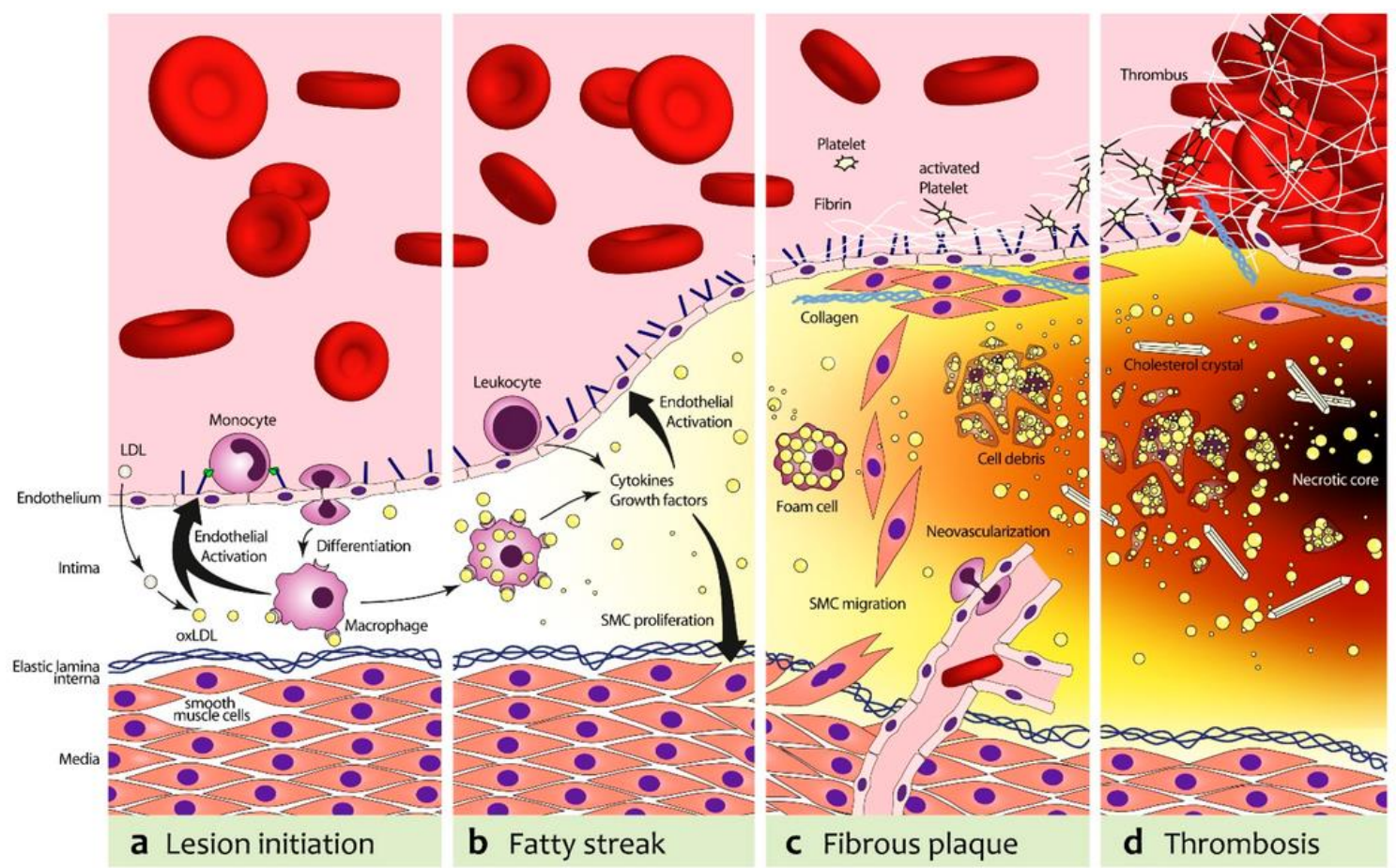

Figure 4. Pathogenesis of atherosclerosis.

(Steinl DC, Kaufmann BA (2015) Ultrasound imaging for risk assessment in atherosclerosis. Int J Mol Sci. 16:9749-9769. doi:10.3390/ijms16059749.)

However, when a plaque would grow big enough to limit the lumen diameter significantly, remodelling of the artery takes place to maintain a nearly normal lumen and additional, hypoxia-induced formation of collaterals will occur. Therefore, a close to $100 \%$ stenosis is possible almost with no symptoms (in case of well-developed collaterals). Neovascularization is common in more advanced, high risk atherosclerotic plaques and is a hallmark of persisting inflammation in site. Through contributing to extravasation of inflammatory cells and erythrocytes, these new leaky and fragile microvessels are connected to rapid plaque development [22,23].

Loss of smooth muscle cells by apoptosis or necrosis results in a thin, fragile fibrous cap and consequently leads to the development of a rupture-prone vulnerable/unstable plaque that is characterized by a large lipid core with high macrophage content and microvessel density, intraplaque hemorrhage and superimposed thrombus formation. In contrast, stable plaques have a smaller lipid core with a significantly lower macrophage content, fewer microvessels and a thicker fibrous cap; therefore, rupture and superimposed thrombus formation is not characteristic of them (Fig. 5) [24]. 

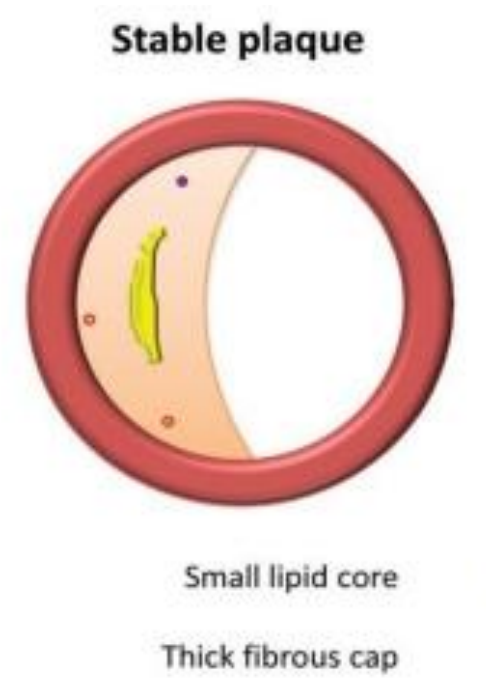

Low macrophage content

Low microvessel density

No Intraplaque hemorrhage

No cap rupture, no superimposed thrombus

\section{Unstable, ruptured plaque}

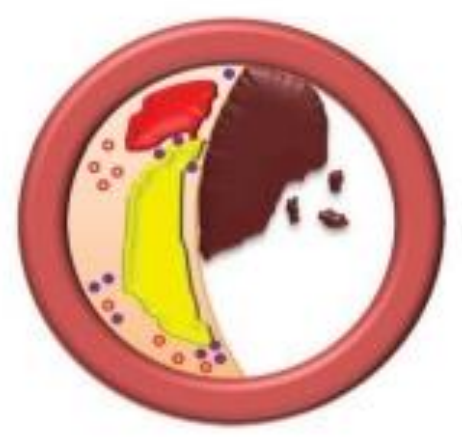

Large lipid core

Thin fibrous cap

High macrophage content

High microvessel density

Presence of Intraplaque hemorrhage

Cap rupture and superimposed thrombus

Figure 5. Schematic overview of a stable atherosclerotic plaque (left) and an unstable atherosclerotic plaque (right).

(W van Lammeren G, L Moll F, Borst GJ, de Kleijn DP, P M de Vries JP, et al. (2011) Atherosclerotic plaque biomarkers: beyond the horizon of the vulnerable plaque. Curr Cardiol Rev 7:22-27. doi:10.2174/157340311795677680.)

Although highly rupture-prone plaques responsible for most acute coronary syndromes are typically larger than those found in the background of stable angina, narrowing of the arterial lumen is usually more predominant in stable angina owing to the slow, constructive remodelling of the vessel wall [25].

Plaques commonly calcificate with aging; degree of this calcification is considered as a traditional marker of coronary plaque burden and linked to the disease prognosis [26]. In acute coronary syndromes, culprit lesions seem to be less calcified compared to plaques causing stable angina $[27,28]$.

Endothel dysfunction also contributes to the formation of a prothrombic environment that favours platelet activation and fibrin deposition onto the inflamed endothelium. Further destabilization of the plaque leads to the rupture of the weakened fibrous cap and ends in the formation of atherothrombus: the released extracellular matrix molecules initiate coagulation 
and platelet recruitment $[29,30]$. Plaque rupture is common and it is the most frequent cause of coronary artery thrombosis; although mostly nonfatal, it contributes substantially to rapid development of the lesion [18].

\subsection{Roles of neutrophils and monocytes in coronary artery disease}

Although neutrophils have received only a little attention in connection with atherosclerosis until lately, it is becoming widely-known by now that they participate actively not only in the very onset, but also in the progression of atherosclerosis [31-34]. They take part in early atherosclerosis through the release of myeloperoxidase form their azurophilic granules and thus, contribute to the proatherogenic oxidative modification of LDL [33]. Furthermore, neutrophils promote monocyte/macrophage recruitment into the lesion and take part in the thinning of the fibrous cap and formation of a vulnerable plaque both directly (e.g. via their derived proteinases) and through activating macrophages [32]. Interestingly, it was found that granulocytes are already primed in hyperlipidemic patients and any second trigger may fully activate them; this results in the release of superoxide radicals and inflammatory mediators that contributes to oxidative stress and inflammation, hence they can be considered as a risk factor for promoting atherosclerosis [35]. Moreover, alfa-defensins, which are neutrophil peptides, could also take part in cardiovascular inflammation [36].

Several studies showed that high neutrophil count or neutrophil-to-lymphocyte ratio is associated with coronary stenosis, and may predict CAD and major adverse cardiac events such as myocardial infarction [37-42]. Neutrophil activation is widely observed in unstable angina $[43,44]$, however, the state of neutrophils in stable angina pectoris patients appears to be controversial [31,45] and requires more investigation. Activation state of neutrophils can be assessed by detecting cell surface lactoferrin, L-selectin and/or CD15 parameters, as their increased appearance on the cell surface can be considered as a sign of cell activation. From these, only lactoferrin can be considered as a specific neutrophil activation marker, its appearance on neutrophil cell surface and in blood plasma reflects degranulation that leads to its release from secondary granules to the circulation following activation [46-48].

Lactoferrin is an iron-binding glycoprotein that can be characteristically found in the secondary granules of the neutrophil granulocytes, in addition to blood plasma and several body

fluids. Beside its antibacterial, antifungal and antiviral activities, it takes part in 
immunomodulation: it modulates the maturation, migration and functions of immune cells. Lactoferrin may act as a cytokine-like proinflammatory factor and it can induce the expression of adhesion molecules on the surface of endothelial cells [49].

L-selectin is a cell-cell adhesion receptor that is continually expressed in leukocyte plasma membrane and takes part in the regulation of early leukocyte trafficking, for example in neutrophil rolling. Since the cell surface appearance of this adhesion receptor can undergo dynamic changes upon leukocyte activation, therefore, it can be used as a marker of the granulocyte activation state [50].

CD15 (or Lewis X) is a carbohydrate adhesion ligand for selectins present on the plasma membrane and in the azurophilic granules of mature neutrophils; it is also expressed by several other leukocytes and epithel cells [51]. Activation of neutrophils induces its translocation from intracellular position to the cell surface [52], it takes part in the cell-cell interactions, the phagocytosis and the respiratory burst of neutrophils [51].

As mentioned above, activated monocytes and macrophages have a central role in the initiation and progression of atherosclerosis through foam cell formation and inflammatory factor production [53,54]. Monocyte count may predict the severity of atherosclerotic stenosis and coronary plaque progression in acute coronary syndromes [41,55]. Moreover, Waterhouse et al. (2008) suggested that of all white blood cell types, monocyte count has the strongest positive and independent relationship with CAD risk in asymptomatic adults [56]. According to a recent paper, decreased lymphocyte-to-monocyte ratio - i.e. an elevated monocyte-tolymphocyte ratio (MLR) - was associated with the prevalence of CAD and prior myocardial infarction, and represents a high risk for critical limb ischemia [57]. Lymphopenia (low lymphocyte count) is associated with increased risk of developing myocardial infarction and long-term all-cause mortality in patients with acute chest pain but without other diagnostic criteria for major cardiac events [58].

Interestingly, while an extensive literature can be found on the neutrophil-tolymphocyte ratio in connection with CAD, only very few papers were published on the clinical importance of the monocyte-to-lymphocyte ratio in atherosclerosis and especially in CAD. 


\subsection{Psychosocial stress and coronary artery disease (psycho-neuro-immunology)}

Psychosocial stress (e.g. social isolation, workplace stress or childhood abuse) and depression are becoming increasingly recognized as major risk factors for incident coronary artery disease and as independent predictors of poor cardiac prognosis [4,8,59-62]. While social isolation and low social support are known as risk factors for developing $\mathrm{CAD}$, low scores in meaning of life is associated with increased risk of CAD mortality [59,62-64]. It is worth noting that stress could result in developing both depression and CAD [60].

Psycho-neuro-immunology (PNI) is a relatively new field of research investigating the interactions between stress, the nervous and immune system and health outcomes [65]. When facing with any kind of physical or emotional stressors, organisms respond with the 'general adaptation syndrome' characterized by Selye [66]. This adaptation is governed primarily by the sympathetic-adrenomedullary system (SAM/SAS) and the hypothalamic-pituitaryadrenocortical (HPA) axis (Fig. 6) and leads to quantitative and qualitative alteration in immune functions $[67,68]$. In stress response, the activity of the sympathetic nervous system (SNS) results in the release of catecholamines: adrenaline (epinephrine) and, to a lesser extent, noradrenaline (norepinephrine) from the adrenal medulla. Furthermore, a direct connection or 'hard-wiring' also exists between the SNS and lymphoid organs [69]. The other principal system involved in stress state is the HPA axis, which acts via corticotropin-releasing hormone (CRH), adrenocorticotropic hormone (ACTH) and cortisol.

Although, cortisol and its analogues are well-known and widely used for their antiinflammatory and immunosuppressive effect, morning plasma cortisol levels were found to correlate with the severity of coronary atherosclerosis in US Air Force aircrew members and in patients with suspected CAD [70,71]. In addition, Hamer et al. reported that a heightened cortisol response to psychosocial stress associated with coronary artery calcification in healthy individuals [72]. 


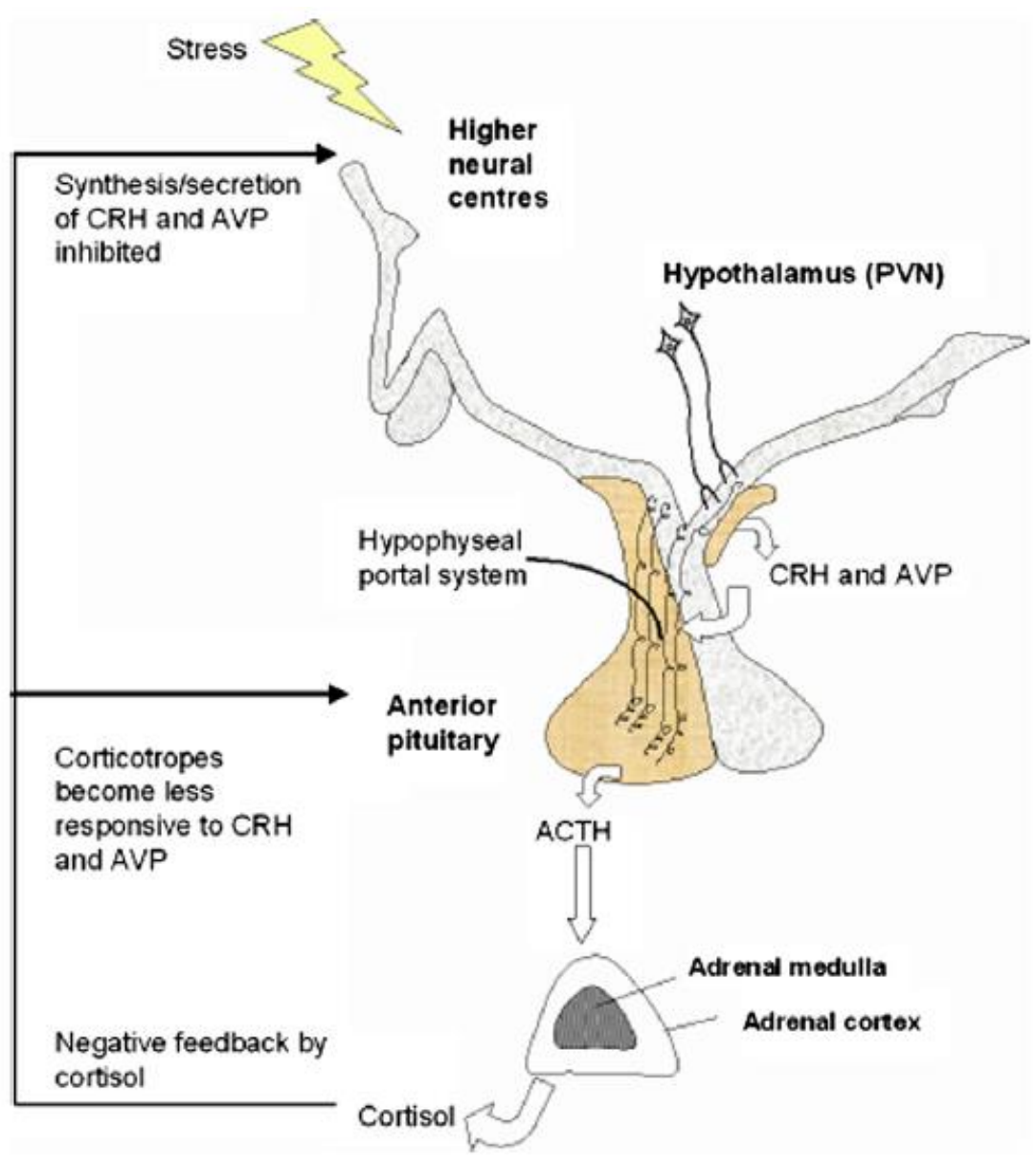

Figure 6. Schematic representation of the hypothalamo-pituitary adrenal (HPA) axis (with its negative feedback regulation).

(Tilbrook AJ, Clarke IJ (2006) Neuroendocrine mechanisms of innate states of attenuated responsiveness of the hypothalamo-pituitary adrenal axis to stress. Front Neuroendocrinol 27:285-307. doi:10.1016/j.yfrne.2006.06.002.)

Among the wide range of cell surface receptors, neutrophils have specific glucocorticoid receptors as well, that can affect their behaviour and homeostasis [32,34,73]. In fact, not only neutrophils, but the majority of the immune cells (e.g. monocytes) have stress hormone receptors (e.g. adrenergic receptors). Several stress hormones (e.g. glucocorticoids, catecholamines, $\mathrm{CRH}$ ) exert immunomodulatory effects that can result in cytokine production, such as interferon- $\gamma($ IFN- $\gamma$ ), various interleukins (IL-1, IL-2, IL-6) and tumour-necrosis factor (TNF) by these target cells [68]. In turn, these cytokines may act back on the hypothalamus and may augment its activity even more; especially IL-6 was found to be a highly potent stimulator of HPA axis [60,67,74]. Merging the above discussed effects: psychological, neuroendocrine and inflammatory systems could interact with each other. As it could be seen, in the psycho- 
neuro-inflammatory network of atherosclerosis, neutrophils and monocytes/macrophages probably have fundamental roles: these leukocytes may potentially promote the initiation and progression of CAD by translating the activation of neuroendocrine system into inflammation in stressed (and hyper-/dyslipidemic) conditions.

Thus, not surprisingly, psychological stress was found to lead to the activation of neutrophils, while relaxation resulted in its attenuation in anxious patients and even in healthy individuals [75-77]. It is known that patients with acute coronary syndrome are usually highly stressed, experience anger or depression; moreover, stable CAD patients also feel tension before or during PCI [78]. Neutrophil activation state, however, has been largely unexplored from the aspect of psycho-neuroendocrine background in patients undergoing PCI.

Similarly, only few studies have investigated associations between monocyte counts/fractions and psychological factors in CAD. Gidron et al. (2003) found that an elevated monocyte fraction was related to lower social support and more life event stress in patients with acute coronary syndrome [79]; however, our knowledge in connection with stable CAD is still incomplete from the PNI aspect.

Chromogranin $\mathrm{A}(\mathrm{CgA})$ is an acidic protein that is co-stored and co-released with catecholamines and produced by the diffuse endocrine and neuroendocrine system, its increased plasma levels could indicate general neuroendocrine overactivity including elevated sympathoadrenal activity [80,81]. Additionally, plasma $\mathrm{CgA}$ is considered as an independent predictor of mortality and heart failure in and after acute CAD, which is due to its production by the overloaded myocardium [82]. Recently, salivary CgA level has been suggested as a decent biomarker of psychosocial stress [83,84], anxiety [85-87] and depression [87,88].

\subsection{Blood plasma inflammatory factors in CAD}

Cytokines are small proteins that have a central importance in the PNI system. Proinflammatory cytokines (especially IL-1, IL-6 and TNF- $\alpha$ ) take part not only in the process of atherosclerosis and CAD, but also in inducing stress and depression. [59,89]. IL-6, which is produced mostly by monocytes, lymphocytes, fibroblasts and endothelial cells [90], is a main regulator of the systemic inflammatory response and it is a potent inducer of the acute phase reaction. During this process, the liver synthetizes C-reactive protein (CRP), which is a widely used systemic inflammatory marker in routine laboratory diagnostics. Both IL-6 and CRP are 
considered as major biomarkers of vascular inflammation in CAD [91]. Furthermore, it was shown that plasma IL-6 and CRP are elevated in psychosocial stress and depression [92,93].

Neutrophils produce several kinds of cytokines, such as the proinflammatory IL-1, IL6 and TNF- $\alpha$, and the immunomodulatory IL-8 [94]. On the other hand, they are affected by several cytokines; accordingly, various cytokine/chemokine receptors are present in their plasma membrane [95]. Stenting could trigger rapid, transient neutrophil activation, which is followed by the production of IL-6 and IL-8 in unstable angina [96]. Similarly, cytokine levels may increase after PCI in stable angina, as well [97].

Recent studies showed that LL-37, the only member of the human antimicrobial cathelicidin family, participates in inflammation and atherosclerosis. It can be expressed by monocytes/macrophages, neutrophils, natural killer cells and epithel cells [98-100]. It was found that LL-37 could be produced also by macrophages in atherosclerotic lesions $[98,101]$. LL-37 provides a chemotactic effect: induces angiogenesis via endothel cell activation and inflammatory cell recruitment to the atherosclerotic plaque [102]. However, LL-37 can also be anti-inflammatory according to the microenvironment [103].

Lactoferrin, a specific activation marker of the neutrophil cell surface, can gain access to the blood and can be regarded as a soluble inflammatory marker as well; its appearance in plasma reflects neutrophil activation/degranulation, which results in the release of proinflammatory cytokines in addition to oxygen radicals [46]. 


\section{Aims}

From the aspect of psycho-neuroendocrine background, our knowledge is still incomplete on the activation state of neutrophils in CAD. Since neutrophils have a fundamental role in coronary atherosclerosis, it is considerably important to reveal the possible stress-related regulation of their activation in $\mathrm{CAD}$ patients before and after stenting. While participation of monocytes/macrophages in atherosclerosis is well-known, associations of the monocyte-tolymphocyte ratio with the psycho-neuro-immune network are largely unexplored in CAD.

Therefore, in our studies, we were particularly interested in the PNI characteristics of our CAD patients and in the potential associations among stress markers, depressive symptoms and inflammatory markers with a special focus on neutrophil activation state and the monocyteto-lymphocyte ratio.

More precisely, we set out to the following investigations:

Study I.: in SAP and ACS patients, in connection with PCI (before, directly after and the next day of PCI)

- assessment of the psycho-neuroendocrino-immune state (stress hormones: ACTH and cortisol, and a general inflammatory marker: IL-6; all tested in plasma)

- analysis of the activation state of granulocytes (cell surface markers: L-selectin, CD15 and neutrophil-specific lactoferrin; plasma lactoferrin)

- examination of the possible associations between granulocyte (neutrophil) activation markers and stress markers (plasma ACTH and cortisol)

Study II.: in SAP patients: cross-sectional investigations

- analysis of the associations of the MLR with neuroendocrine markers (cortisol and CgA), inflammatory parameters (IL-6, CRP, LL-37) and psychosocial factors (depressive symptoms and stress-coping scores)

- investigations on further associations of the PNI network: among psychological factors, inflammatory parameters and neuroendocrine markers 


\section{Materials and methods}

\subsection{Patients}

Study participants were recruited into two separate studies. In both studies, all participants gave a written consent to be enrolled in the study. The investigations conformed to the principles of the Declaration of Helsinki. The study protocols were approved in advance by the Regional Medical Ethics Committee (Human Investigation Review Board, University of Szeged, Albert Szent-Györgyi Clinical Centre; Ref. No: 37/2005 (for study I.), Ref. No: 49/B125/2008, 2382 (for study II.)

In study I., patients were eligible for inclusion if they had the characteristic symptoms of either stable angina pectoris (SAP) confirmed by a positive ergometric stress test or acute coronary syndrome (ACS). 25 patients with SAP and 20 patients with ACS were recruited and enrolled; however, our study involved only 41 subjects (21 SAP and 20 ACS) because 4 SAP patients' blood samples were not suitable for processing (as a consequence of blood clotting disturbances and sample handling problems). In the ACS group, 6 patients had unstable angina, 14 were diagnosed with acute myocardial infarction. Additional requirement for inclusion was the presence of at least one significant coronary artery stenosis (diameter $>50 \%$ ), which was demonstrated by coronary angiography as suggested by Videm et al. (2007) [45]. Clopidogrel (600 mg loading dose, 75-150 mg maintenance dose) before and after PCI was administered to all patients, as well as heparin (70-100 IU/kg) during PCI.

During the procedure of PCI, a thin and flexible catheter with an inflatable balloon mounted very close to its tip is inserted into the radial (or femoral) artery and navigated towards the heart, where the critically narrowed coronary artery can be found. When it reaches the site of stenosis, the balloon covered with a stent inflates that compresses the plaque into the coronary wall and restores normal lumen and blood flow. The balloon is then deflated and withdrawn from the coronary system, but the implanted stent stays in the artery and supports the stretched artery wall (Fig. 7). 


\section{Coronary Artery with Stent}

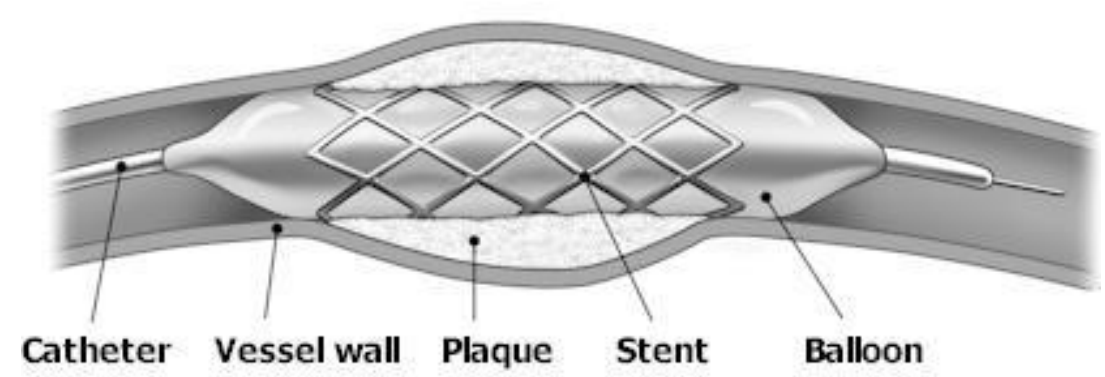

Figure 7. Coronary artery with stent

(http://www.cpmc.org)

In study II., 23 patients with stable CAD participated. They had to have confirmed significant stenosis (diameter $>50 \%$ ) at least in two major coronary arteries to be included. The severity of heart failure symptoms of the patients was graded according to the New York Heart Association functional classification (NYHA, grades I-IV). Three (13\%), four (17\%), and five $(22 \%)$ patients were in NYHA groups I, II, and III, respectively.

Table 1. General characteristics of the patient groups

\begin{tabular}{|l|c|c|c|c|}
\hline \multirow{2}{*}{\multicolumn{1}{|c|}{ Variable }} & \multicolumn{3}{|c|}{ Study I. } & Study II. \\
\cline { 2 - 3 } & SAP & ACS & \multirow{2}{*}{$\mathrm{p}$} & stable CAD \\
\cline { 2 - 3 } & $\mathrm{n}=21$ & $\mathrm{n}=20$ & & $\mathrm{n}=23$ \\
\hline Age (y) & $64.1 \pm 10.3$ & $63.2 \pm 13.6$ & 0.81 & $62.87 \pm 10.59$ \\
\hline Male gender (n, \%) & $13(61.9)$ & $14(70.0)$ & 0.74 & $18(78)$ \\
\hline BMI $\left(\mathrm{kg} / \mathrm{m}^{2}\right)$ & n.a. & n.a. & n.a. & $28.80 \pm 4.00$ \\
\hline Current smoking (n, \%) & n.a. & n.a. & n.a. & $2(9)$ \\
\hline Diabetes mellitus type II (n, \%) & $5(23.8)$ & $6(30.0)$ & 0.73 & $8(35)$ \\
\hline Hypertension (n, \%) & $16(76.2)$ & $16(80.0)$ & 1.00 & $20(87)$ \\
\hline Statins use (n, \%) & $16(76.2)$ & $15(75.0)$ & 1.00 & $21(91)$ \\
\hline Antihypertensives use (n, \%) & $21(100)$ & $17(85.0)$ & 0.11 & $22(96)$ \\
\hline Sedatives use (n, \%) & $7(33.3)$ & $7(35.0)$ & 1.00 & $9(39)$ \\
\hline Proton pump inhibitors use $(\mathrm{n}, \%)$ & n.a. & n.a. & n.a. & $16(70)$ \\
\hline
\end{tabular}

SAP: stable angina pectoris, ACS: acute coronary syndrome, CAD: coronary artery disease, n.a.: not available. Age and BMI are shown as mean \pm SD. 
In both studies, exclusion criteria covered prior bypass surgery, cardiogenic shock, malignancy (e.g. prostate cancer), immunological disorders (e.g. rheumatoid arthritis, chronic atrophic gastritis Type A), acute inflammations, treatment with immunosuppressive or antiinflammatory drugs (exception: low-dose aspirin, which was administered to all patients), recent major trauma or surgery, drug or alcohol abuse and poor mental function. Additional exclusion criteria in study II. was impaired renal function. None of the patients took antidepressants and/or H2-receptor blocker medication. Patient characteristics of both studies are shown in Table 1.

\subsection{Blood sampling and laboratory methods}

Fasting venous blood samples were collected immediately before PCI (pre-PCI), immediately after PCI (post-PCI) and on the following day of PCI (1d-PCI) between 8-9 a.m. in study I.; in study II. samples were obtained 1 day before PCI between 7-10 a.m. The mean time of PCI was 45 min (range: 30 min-1 hour).

Blood collection for routine blood analysis was carried out at admission for the acute patient group (study I.); and before admission for stable patients (in both studies) to check the exclusion criteria. Creatine kinase (CK) values were measured in 1d-PCI samples in both studies to monitor heart attack in acute patients and to observe the occurrence of acute procedural myocardial infarction in stable patients. Cardiac troponin $\mathrm{T}$ was measured with a qualitative dry chemistry test using heparinized whole blood and was checked visually.

Blood cell counts, total cholesterol, triglycerides, HDL-cholesterol and high sensitivity CRP (in study II. only) were determined in automatic analyzers and/or with commercial kits (Roche); LDL-cholesterol was calculated according to the Friedewald formula. Reference ranges: total leukocyte count: $3.9-11.110^{9} /$, neutrophil fraction: $44.0-68.0 \%$, neutrophil count: 1.7-6.1 $10^{9} / 1$, monocyte fraction: 5-7\%, monocyte count: $0.2-0.610^{9} / 1$, lymphocyte fraction: $27-$ 34\%, lymphocyte count: 1.0-3.2 10 $/ \mathrm{l}$, total cholesterol: $<5.2 \mathrm{mmol} / \mathrm{l}$, triglycerides: $<2.0$ mmol/l, HDL-cholesterol: >1.0 mmol/l, LDL-cholesterol: <3.0 mmol/l, CK activity: <200 U/l, CRP: $<5.0 \mathrm{mg} / \mathrm{l}$. For study II., monocyte count was divided by the lymphocyte count to get the MLR; since no reference interval was available for it, we used the ratio of the median values of the monocyte and lymphocyte reference ranges (counts) as reference: 0.19 . 


\subsubsection{Special plasma marker assays}

We collected blood into cooled $\left(4^{\circ} \mathrm{C}\right)$ EDTA-Vacutainer tubes, and stored plasma in aliquots in $-80^{\circ} \mathrm{C}$ for special analyses.

\subsubsection{Neuroendocrine (stress) markers}

In study I., ACTH and cortisol were measured as stress markers, in study II., in addition to cortisol, $\mathrm{CgA}$ was applied as a neuroendocrine marker.

ACTH concentrations were measured by a chemiluminescent assay (LKAC1, Immulite 1000; Siemens Healthcare Diagnostics, UK; intra- and inter-assay CVs: $6.1 \%$ and 9.4\%, respectively; reference range: $<60 \mathrm{pg} / \mathrm{ml}$ ). Cortisol was tested by radioimmunoassay (DSL2100; Diagnostic Systems Laboratories, Webster, TX; intra- and inter-assay CVs: $<12 \%$; reference range: $160-620 \mathrm{nmol} / \mathrm{l})$. Chromogranin A was analysed with a radioimmunoassay kit (CGA-RIA CT; CIS Bio International, Gif-sur-Yvette, France; intra- and inter-assay CVs: $<7 \%$; reference range: $23-153 \mathrm{ng} / \mathrm{ml}$ ).

\subsubsection{Inflammatory parameters}

In study I., lactoferrin and IL-6, in study II., IL-6 and LL-37 plasma concentrations were determined as special inflammatory markers (in blood plasma).

For the assessment of lactoferrin, an 'in-house' ELISA kit was applied, which was developed according to Antonsen et al. (1993) [104]. Briefly, microplate wells were coated for overnight with rabbit anti-human lactoferrin antibodies $\left(3.7 \mathrm{mg} / \mathrm{l}\right.$; Dako, Denmark) at $4^{\circ} \mathrm{C}$. After two washing steps, duplicate plasma samples were applied (ten-fold dilution in wells), and incubated for $1 \mathrm{~h}$ at room temperature. After washing five times, anti-lactoferrin-peroxidase was added into wells and incubated for another $1 \mathrm{~h}$ at room temperature (4000-fold dilution in wells). The conjugate was prepared from Dako antibody and from horseradish peroxidase (Calbiochem, USA) according to Wilson and Nakane (1987) [105]. After further washing steps, TMB (BD Biosciences, USA) was used to develop plates and $4 \mathrm{~N}$ sulphuric acid to stop the reaction. Human lactoferrin (Sigma, USA) was used as standard, the stock solution ( $40 \mu \mathrm{g} / \mathrm{ml}$, 
in TBS-glycerol, stored at $-20^{\circ} \mathrm{C}$ ) was diluted in TBS-Tween prior application. Reference range/median for plasma lactoferrin: 40-200/90 $\mathrm{ng} / \mathrm{ml}$ [104].

IL-6 was assayed by a commercial ELISA module set containing matched antibody pairs (BMS213/2MST; Bender MedSystems GmbH, Vienna, Austria) in study I., while in study II. a high sensitivity ELISA kit (BMS213HS; Bender MedSystems GmbH, Vienna, Austria; intra- and inter-assay CVs: $4.95 \%$ and $6.0 \%$, respectively; reference range: $<8.7 \mathrm{pg} / \mathrm{ml}$ ) was used. LL-37 concentration was measured by an ELISA kit (HK321, Hycult Biotech Inc., Uden, the Netherlands; intra- and inter-assay CVs: $<10 \%$; reference range: $25-250 \mathrm{ng} / \mathrm{ml}$ ).

\subsubsection{Determination of surface granulocyte activation markers (study I.)}

L-selectin, CD15 and lactoferrin surface granulocyte activation markers were measured by flow cytometry using an indirect immunofluorescent method; the process of labelling and haemolysis was started within 2 hours after blood collection. The protocol was performed according to Keresztes et al. (2007) [76]. Since it is known that cell isolation techniques activate leukocytes, thus, whole blood samples were used. Whole blood aliquots $(100 \mu \mathrm{l})$ were treated with $(20 \mu \mathrm{l})$ saturating concentrations of a polyclonal anti-lactoferrin antibody (200 mg/l, ICN), a monoclonal anti-L-selectin (47 mg/l, Dako) or a monoclonal anti-CD15 antibody $(8.5 \mathrm{mg} / \mathrm{l}$, Dako). Negative controls contained normal rabbit serum or isotype-specific normal mouse Ig (Dako). After incubation for $20 \mathrm{~min}$ at room temperature and washing two times with Hank's solution, sedimentation was carried out in Eppendorf tubes (using Heraeus Biofuge pico, 13000 rpm). Within 1 hour after drawing blood, immunostaining was initiated with appropriate secondary antibodies: anti-rabbit $(10 \mathrm{mg} / \mathrm{l})$ or anti-mouse $(20 \mathrm{mg} / \mathrm{l})$ antibodies labelled with FITC (Dako) were added to the blood cells (20 $\mu$ l aliquots of reagents to $180 \mu 1$ aliquots of blood cells). Following incubation and further washing steps (carried out as before), hemolysis was performed using a lysis buffer (Lysing kit; Biodesign, USA). One millilitre of lysis reagent was added to a $100 \mu \mathrm{l}$ aliquot of blood cells; after vigorous vortexing and 15 min incubation, sedimentation (6000 rpm) and a washing step (Hank's solution) followed. Finally, blood cells were resuspended in $200 \mu$ l Hank's buffer.

Analyses were performed using a FACStar Plus Becton Dickinson equipment; only the granulocyte population was tested from leukocytes. Granulocytes were gated according to their 
typically high forward- (FS) and side-scatter (SS) characteristics; they could be easily separated from lymphocytes and monocytes - the two other leukocyte populations in the flow cytometry analysis. (The side scatter is associated with the granularity of the cell; the forward scatter is related to the diameter of the cell.) Granulocytes with lactoferrin expressed on their surface were identified as activated neutrophils, because lactoferrin is specific for neutrophils and can get the cell surface only after degranulation followed by cell activation [46-48]. 10000 events/tube were recorded in granulocyte populations. Standard equipment calibrations preceded all measurements and proper negative controls were used to set the background intensity level before each detection series were determined. Ratios of labelled granulocytes (\% of granulocytes bearing labelled markers on cell surface) and mean fluorescence intensities (MFI, associated to mean quantity of labelled molecules/cells) were calculated. Figure A8 (annex section) shows examples of the performed flow cytometry analyses.

\subsection{Psychosocial measures (study II.)}

We used the Hungarian version of the 21-item Beck Depression Inventory (BDI) to measure the severity of the depressive symptoms of patients $[106,107]$. Study participants rate every item on a scale from 0 to 3 . Total score results may show no clinically relevant depressive symptoms (0-9), mild (10-18), moderate (19-25) or severe (25-63) depressive symptoms.

We applied Rahe's Brief Stress and Coping Inventory (BSCI, a multidimensional selfdescribing device) to evaluate everyday stress level and coping capacity [108]. In order to receive the global stress coping score, total stress score is subtracted from total coping score. According to the validated Hungarian version of the inventory, total scores may represent worrisome/non-sufficient (-15 to -5 ), sufficient ( -4 to -1$)$, good (0 to 4$)$ and very good (5-15) global stress-coping capacity [109]. Both stress and coping scales contain 5 subscales. Coping subscales measure health habits, social support, responses to stress, life satisfactions, and purpose and connection. Specifically, the social support subscale measures the degree of a subject's social network; the purpose and connection subscale (also called meaning of life) shows to what extent participants feel their life 'worth living' and it includes also a component of spirituality/religious attitude [64]. Subscale scores range between 0 and 14 (social support) or 16 (life meaning) and indicate worrisome/non-sufficient (0-7), sufficient (8-10), good (1113), or very good/excellent (14-16) coping with psychosocial stress. 


\subsection{Statistical analysis}

Continuous variables are presented as mean \pm standard deviation (SD); two-sample Student's t test was used to compare the general characteristics of the groups in study I. Categorical variables are shown as number of subjects (n) and percentage (\%), and the groups were compared by Fisher's exact test. Data distribution was checked by Kolmogorov-Smirnov statistics; data that did not show normal distribution were log-transformed before further analyses (ACTH in study I., the MLR, CRP and BDI score in study II.). All tests were twosided with a significance level of $\mathrm{p}<0.05$.

In study I., figures are presented as mean \pm standard error of the means (SEM). Twoway repeated measurements ANOVA was applied for over time comparisons (pre-PCI, postPCI, and 1d-PCI samples). Two main factors were considered in the analysis: the time (prePCI, post-PCI and 1d-PCI) as within group ('intragroup') factor for repeated measurements and the two subject groups as between-subject ('intergroup') factor for independent samples; $p$ values were for overall intragroup $\mathrm{p}$, between groups/intergroup $\mathrm{p}$, and one for their interaction. Significant value of interaction and special cases, when its value was relatively low: $0.3>p>0.05$ (the presence of interaction was assumed) allowed also between-group (SAP vs ACS) comparisons at each time point. Significant value of the separate intragroup $\mathrm{p}$ allowed further pairwise comparisons of the repeated measurements within each group. All the pairwise comparisons are modified t-tests, these and the separate intragroup p-s were calculated by the software based on estimated marginal means. To keep the familywise type I error rate on $\alpha=0.05$ level, we used the step-down Bonferroni adjustment for multiple comparisons. We used a mixed model to assess the change in the variances of repeated measures over time and at the same time, to take account of the possible correlation of the responses measured on the same subject. We chose a general unstructured form for within-subject variance covariance matrix, heterogeneity of group-variances was also taken into account.

Spearman's coefficient of correlation and its significance were calculated to assess the relationship between the stress hormones and selected inflammatory markers. Statistical analyses were performed using IBM-SPSS (version 15.0, SPSS Inc., Chicago, IL, USA) and SAS (v9.1, SAS institute, Cary, NC) softwares.

In study II. we first calculated the necessary sample size to achieve clinically meaningful correlation coefficients of $r \geq 0.6$ between variables of interest with a power of $80 \%$ and $\alpha=0.05$ 
level of significance. The power analysis resulted a sample size of $n=19$ patients. For the compensation of potential dropouts, we recruited 24 patients; as one patient was transferred to another hospital, 23 subjects left to participate in our study. According to Cohen's guidelines, effect size of correlation coefficients ( $r$ ) with values of $0.1,0.3$ and 0.5 could be interpreted as small, medium and large, respectively [110]. Correlation coefficients with values $\geq 0.6$, therefore, highly likely show existing associations between the tested variables regardless of pvalues, and even when performing multiple tests.

In this still growing field of research, Bonferroni adjustment carries the risk of deriving truly important differences nonsignificant [111]. We decided not to adjust p-values for multiple comparisons as a consequence of that we hypothesized significant associations between the MLR and psychological factors, neuroendocrine and inflammation markers [112]. Secondary hypotheses were also defined for significant association amongst psychosocial factors, neuroendocrine and inflammation markers (except the MLR).

We used bivariate and multivariate Pearson correlation analyses with adjustment for age, sex and BMI in order to examine the associations of the MLR with psychosocial factors, neuroendocrine and inflammatory markers; and similarly, to estimate the associations among psychosocial factors and neuroendocrine and inflammatory markers. Additionally, we adjusted CgA models for intake of proton pump inhibitors and NYHA grades, as this medication could elevate $\mathrm{CgA}$ levels and in heart failure $\mathrm{CgA}$ could also be elevated [82,113]. Models with BDI scores were additionally corrected for sedative use, as intake of sedatives influenced significantly on BDI scores among our patients. Statistical analyses were carried out using IBMSPSS software (version 20.0, SPSS Inc., Chicago, IL, USA).

\section{Results}

\subsection{Routine blood data}

In study I., we found that in the ACS group, patients had slightly elevated LDLcholesterol levels and markedly increased CK activity. Other significant between-group differences were not revealed and all other tested laboratory parameters were within the normal range. 
In study II., we found that the monocyte fraction $(8.68 \pm 2.97 \%)$ exceeded the maximal reference value (7\%), and monocyte count came close to the upper reference limit; in the same time, lymphocyte fraction approached the minimal reference value. Therefore, the MLR yielded a considerably high value $(0.32 \pm 0.14)$ and exceeded the predefined reference value of 0.19 . We observed normal values for the other white blood cells and blood lipids except for slightly elevated triglyceride levels. CRP was close to its upper reference limit $(4.93 \pm 5.27 \mathrm{mg} / \mathrm{l})$. Routine blood data of the patients are shown in Table 2.

Table 2. Routine blood data of the patient groups (Variables are means $\pm \mathrm{SD}$ )

\begin{tabular}{|l|c|c|c|c|}
\hline \multirow{2}{*}{\multicolumn{1}{|c|}{ Variable }} & \multicolumn{2}{c|}{ Study I. } & Study II. \\
\cline { 2 - 3 } & SAP & ACS & \multirow{2}{*}{$\mathrm{p}$} & stable CAD \\
\cline { 2 - 3 } \cline { 5 - 5 } & $\mathrm{n}=21$ & $\mathrm{n}=20$ & $\mathrm{n}=23$ \\
\hline Total leukocyte count (G/l) & $7.02 \pm 1.41$ & $8.37 \pm 3.02$ & 0.08 & $6.73 \pm 1.22$ \\
\hline Neutrophil granulocyte count (G/l) & n.a. & n.a. & n.a. & $4.05 \pm 1.04$ \\
\hline Neutrophil granulocyte fraction (\%) & $62.62 \pm 7.46$ & $65.83 \pm 8.18$ & 0.2 & $59.70 \pm 6.86$ \\
\hline Monocyte count (G/l) & n.a. & n.a. & n.a. & $0.58 \pm 0.23$ \\
\hline Monocyte fraction (\%) & n.a. & n.a. & n.a. & $8.68 \pm 2.97$ \\
\hline Lymphocyte count (G/l) & n.a. & n.a. & n.a. & $1.90 \pm 0.41$ \\
\hline Lymphocyte fraction (\%) & n.a. & n.a. & n.a. & $28.82 \pm 6.30$ \\
\hline Cholesterol (mmol/l) & $4.34 \pm 1.28$ & $5.01 \pm 1.10$ & 0.11 & $4.30 \pm 1.35$ \\
\hline HDL-Cholesterol (mmol/l) & $1.29 \pm 0.45$ & $1.30 \pm 0.41$ & 0.95 & $1.18 \pm 0.32$ \\
\hline LDL-Cholesterol (mmol/l) & $2.36 \pm 1.04$ & $3.13 \pm 1.04$ & $0.043 *$ & $2.27 \pm 0.94$ \\
\hline Triglycerides (mmol/l) & $1.60 \pm 0.62$ & $1.35 \pm 1.01$ & 0.36 & $2.04 \pm 1.25$ \\
\hline CK (U/l) & $175.0 \pm 286.6$ & $669.8 \pm 715.7$ & $0.008^{*}$ & $122.78 \pm 104.69$ \\
\hline CRP (mg/l) & n.a. & n.a. & n.a. & $4.93 \pm 5.27$ \\
\hline
\end{tabular}

SAP: stable angina pectoris, ACS: acute coronary syndrome, CAD: coronary artery disease, n.a.: not available.

Asterisks $(*)$ denote significant differences between SAP and ACS patient groups $(\mathrm{p}<0.05)$.

Exceptions for sample numbers: total cholesterol, triglycerides (SAP: $n=19$, ACS: $n=16$ ); HDLcholesterol (SAP: $n=18$, ACS: n=16); LDL-cholesterol (SAP: $n=18$, ACS: $n=15$, stable CAD: n=22). 


\subsection{Special laboratory assays from blood samples}

\subsubsection{Neuroendocrine markers: cortisol, ACTH, chromogranin A}

In study I., we tested the plasma values of cortisol and ACTH as neuroendocrine markers of stress. Raised plasma cortisol and grossly elevated ACTH levels in ACS patients showed that they were in a stressed state on admittance to the clinic; these values declined dramatically next day after PCI: plasma cortisol approximately halved $(\mathrm{p}<0.01)$ and ACTH value decreased by $85 \%$ ( $\mathrm{p}=0.0001$ ). Cortisol value of SAP patients resided in the normal range and did not change significantly among the observed time points, however, ACTH value also declined substantially in these patients the day after PCI (pre-PCI/1d-PCI: $\mathrm{p}<0.05$, post-PCI/1d-PCI: $\mathrm{p}<0.01)$. Additionally, while the pre-PCI cortisol was considerably higher in the ACS group $(\mathrm{p}<0.05)$, the 1d-ACTH value was significantly higher in the SAP group than in the ACS one $(\mathrm{p}<0.01)$. We could demonstrate significant (statistical) interactions in the case of these stress hormones (cortisol: $\mathrm{p}<0.001$, ACTH: $\mathrm{p}<0.05$ ), this allowed us to compare the two groups.
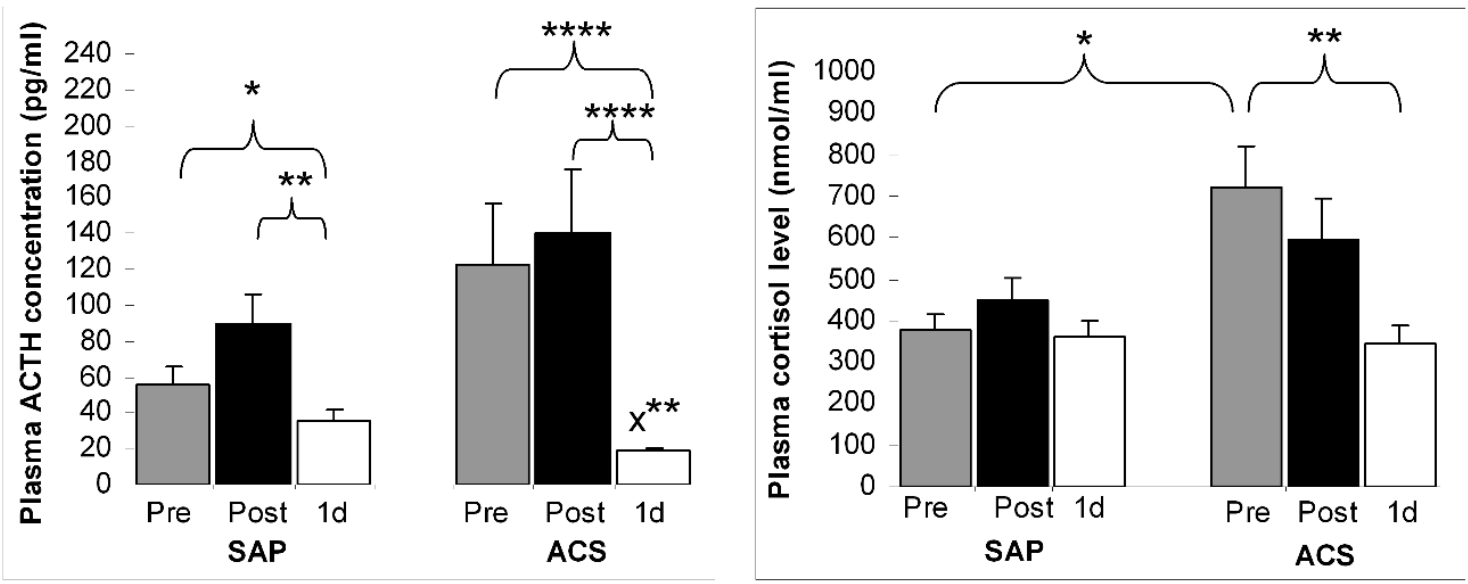

Figure 9. Plasma levels of ACTH and cortisol in CAD patients with stable angina pectoris (SAP, $\mathrm{n}=21$ ) or with acute coronary syndrome (ACS, $n=20)$ taken directly before, directly after and on the following day of percutaneous coronary intervention (PCI) (pre-, post- and 1d-PCI samples: Pre, Post, 1d).

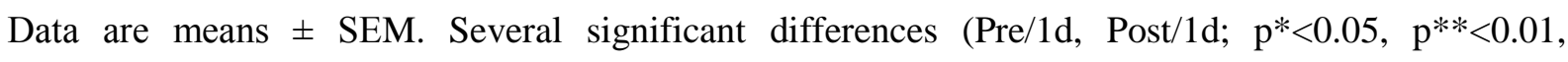

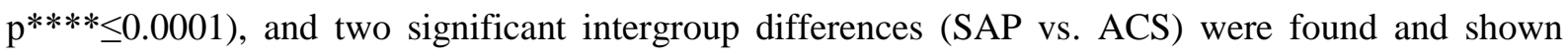

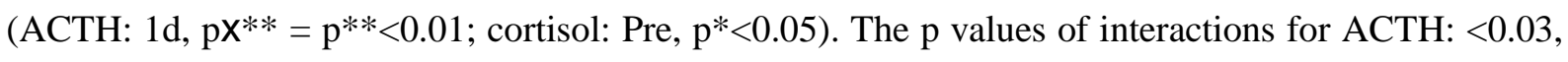
and for cortisol: $<0.001$. 
Pre-, post- and 1d-PCI values of plasma ACTH and cortisol of SAP and ACS patients are shown in Fig. 9, detailed data can be found in Table A3 in the annex section.

In study II., plasma cortisol levels were in the normal range $(294.45 \pm 88.59 \mathrm{nmol} / \mathrm{l})$, similarly as observed in SAP patients in the previous study. $\mathrm{CgA}$ exceeded the upper reference range limit of $153 \mathrm{ng} / \mathrm{ml}(178.81 \pm 105.50 \mathrm{ng} / \mathrm{ml})$ - given according to recent investigations of Glinicki et al. (2015) [114]. Data are presented in Table A4 as well (annex section).

\subsubsection{Special inflammatory markers in plasma: lactoferrin, IL-6, LL-37}

In study I., we employed lactoferrin and IL-6 as plasma inflammatory markers. After a moderate increase in post-PCI samples $(\mathrm{p}<0.001)$, plasma lactoferrin values fell to about $50 \%$ of the post-PCI levels next day after PCI $(\mathrm{p}<0.0001)$ in both groups. Both pre- and post-PCI plasma lactoferrin concentrations exceeded the upper physiological limit of $200 \mathrm{ng} / \mathrm{ml}$. Alteration of plasma IL-6 values followed a different pattern: after a mild increase on the postPCI time point, it showed an about 2-fold elevation in 1d-PCI samples (pre-PCI/post-PCI: p<0.001, post-PCI/1d-PCI: $\mathrm{p}<0.0001)$. Pre-, post- and 1d-PCI values of plasma lactoferrin and IL-6 in SAP and ACS patients are shown in Fig. 10, detailed data can be found in Table A5 in the annex section.
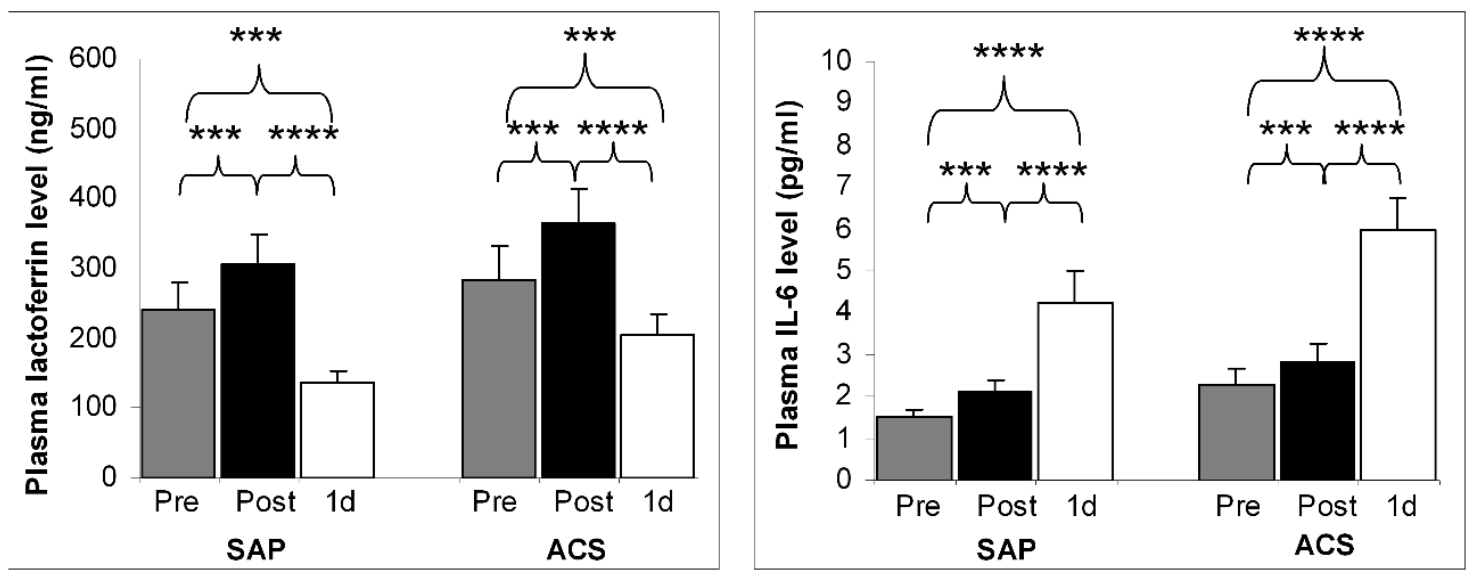

Figure 10. Plasma levels of lactoferrin and IL-6 directly before, directly after and on the following day of PCI in patients with stable angina pectoris (SAP, n=21; IL-6: n=18) or with acute coronary syndrome (ACS, $n=20$; IL-6: $n=19$, except: Post: $n=18$ ).

Data are presented as means \pm SEM; $\mathrm{p}^{* * *}<0.001, \mathrm{p}^{* * * *} \leq 0.0001$. The two patient groups were not statistically different (interaction p values $>0.3$ ). 
Beside the routinely measured plasma CRP, we chose to test IL-6 and LL-37 plasma levels in study II. to assess the general level of inflammation, and found that both of these marker values resided in the normal range (IL-6: $1.83 \pm 1.35 \mathrm{pg} / \mathrm{ml}$, LL-37: $55.31 \pm 12.44$ $\mathrm{ng} / \mathrm{ml}$ ). Data are presented in Table A4 as well (annex section).

6.2.3. Surface activation markers of granulocytes: L-selectin, CD15, lactoferrin in study I.

Concerning L-selectin signal intensity (MFI: mean fluorescence intensity), we found no time- and/or intervention-dependent associated changes. In the SAP group, L-selectin-bearing granulocyte proportion increased slightly during PCI $(\mathrm{p}<0.01)$ and decreased next day to about its initial value $(\mathrm{p}<0.05)$. Similarly, the percentage of CD15-carrier granulocytes raised somewhat during PCI (pre-PCI/post-PCI: $\mathrm{p}<0.05$ ) and showed a gentle decline next day (postPCI/1d-PCI: $\mathrm{p}<0.0001$ ) in both groups. Additionally, the 1d-PCI percentage of the CD15bearing cells decreased below the values measured on the initial time point $(\mathrm{p}<0.01)$ of both groups. CD15 MFI reduced slightly after PCI ( $<<0.05)$ in the ACS group, and furthermore, we could show a difference between groups in its $1 d-P C I$ values $(p<0.05)$.

We observed more remarkable changes in the values of neutrophil-specific surface lactoferrin. Following PCI, the ratios of these lactoferrin-carrying cells fell to roughly half of their post-PCI values (post-PCI/1d-PCI: $\mathrm{p}<0.0001$ ) in both groups. Similarly to the CD15bearing cell ratios, after a slight elevation during PCI $(\mathrm{p}<0.01)$, the proportions of surface lactoferrin-bearing cells were reduced considerably below the pre-PCI values $(p<0.0001)$. Lactoferrin MFI altered less substantially, we found no significant elevation during PCI, however a considerable decline could be seen after PCI in both groups (post-PCI/1d-PCI: $\mathrm{p}<0.001)$. Pre-, post- and 1d-PCI values of the marker-bearing cell ratios and mean fluorescence intensities of granulocytes in SAP and ACS patients are shown in Fig. 11. (Detailed results can be found in Table A6 in the annex section.) 

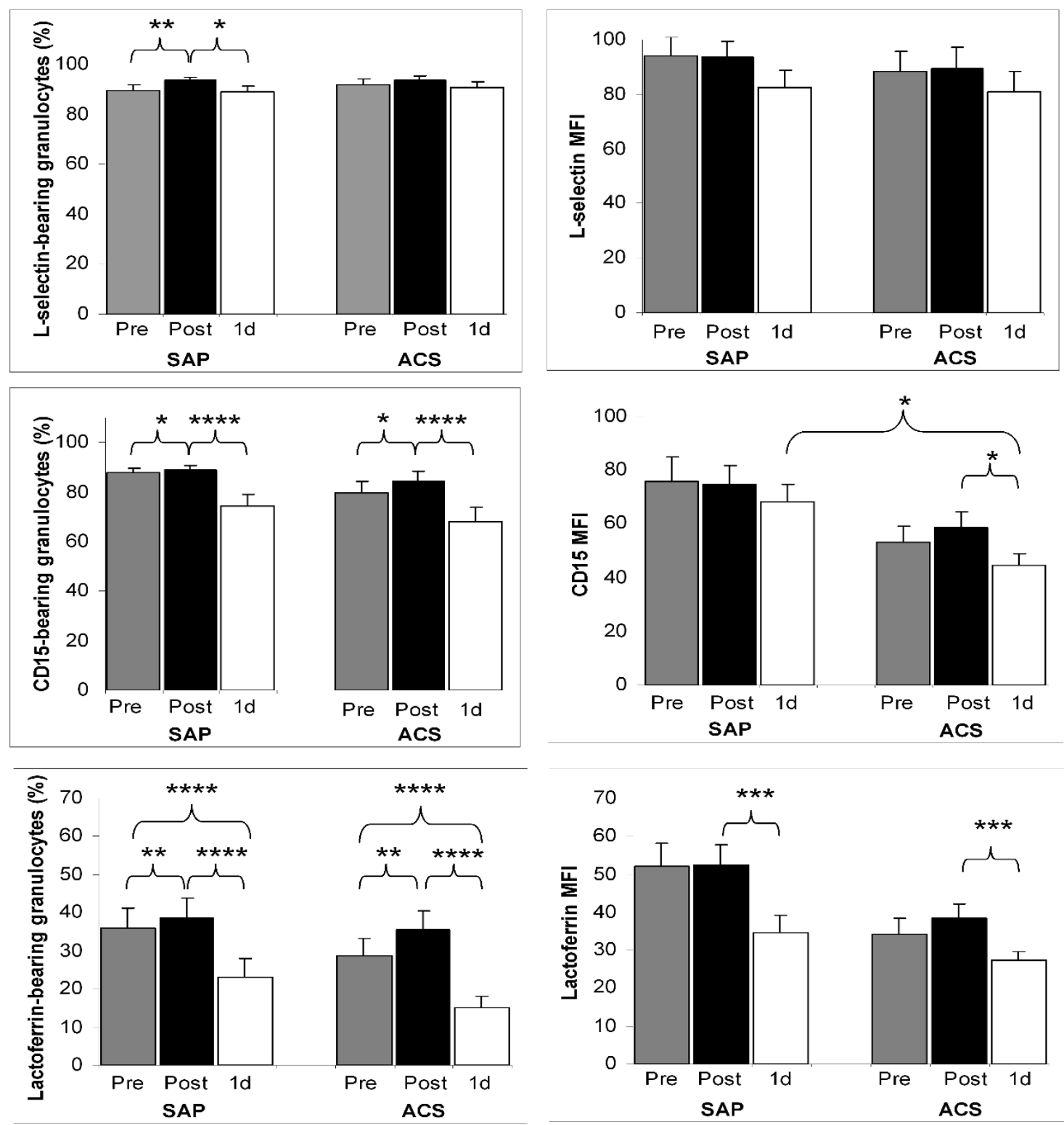

Figure 11. Ratios of marker-bearing cells (\%) and mean fluorescence intensities (MFI) of granulocytes reflecting the cell surface appearance of activation markers directly before, directly after and on the following day of PCI (Pre, Post, 1d) in patients with stable angina pectoris (SAP, $n=21$ ) or with acute coronary syndrome (ACS, $n=20$; exception: 1d values, $n=19)$.

Data are presented as means $\pm \mathrm{SEM} ; \mathrm{p}^{*<0}<05, \mathrm{p}^{* *<0} 0.01, \mathrm{p}^{* * *}<0.001, \mathrm{p}^{* * * *} \leq 0.0001$ 


\subsection{Psychosocial factors in study II.}

Although the average depressive symptom level of the subjects was in the normal subclinical range $(7.4 \pm 9.4)$, seven patients $(30 \%)$ had so high BDI scores that indicated the presence of clinically relevant depressive symptoms (five patients had mild, one patient had moderate and another one had severe symptoms). Interestingly, a subgroup analysis revealed that participants who took sedatives had significantly higher BDI scores than those who did not $(12.8 \pm 12.6$ vs. $3.9 \pm 4.4, \mathrm{p}<0.05)$.

The mean global stress-coping score yielded below zero $(-0.7 \pm 4.2)$, reflecting that the patients had higher stress than coping scores, however, its value was in the 'sufficient' range. Mean social support score was relatively low and close to reach the 'sufficient' range $(7.9 \pm$ 2.4); the meaning of life score was evaluated as 'good' (11.9 \pm 1.9$)$. Data are presented in Table A4 as well (annex section).

\subsection{Correlations}

6.4.1. Correlations between stress hormones (cortisol, ACTH) and selected markers: percentage of lactoferrin-bearing neutrophils, plasma lactoferrin and IL-6 in study I.

As plasma lactoferrin, IL-6 and the ratio of surface lactoferrin-bearing cell values altered the most substantially, we examined the correlations of these inflammatory markers with the stress hormones (Table 7). Plasma ACTH showed inverse associations with the percentage of lactoferrin-carrying neutrophils (SAP: $r=-0.601, \mathrm{p}<0.005$; ACS: $\mathrm{r}=-0.541, \mathrm{p}<0.05$ ) and with plasma lactoferrin in 1d-samples of both SAP and ACS groups (SAP: $r=-0.435, p<0.05$; ACS: $r=-0.609, \mathrm{p}<0.005)$. Furthermore, ACTH associated inversely with IL-6 in the pre-PCI sample of the SAP patient group $(\mathrm{r}=-0.483, \mathrm{p}<0.05)$, and at the post-PCI time point of both groups as well (SAP: $\mathrm{r}=-0.487, \mathrm{p}<0.05$; ACS: $\mathrm{r}=-0.503, \mathrm{p}<0.05$ ). Surprisingly, cortisol correlated positively with plasma lactoferrin in the ACS group before the intervention $(r=0.499, p<0.05)$, but no other significant correlations were observed for this stress hormone in post- or 1d-PCI samples. 
Table 7. Correlations between stress hormones and selected inflammatory markers

\begin{tabular}{|l|c|c|c|}
\hline \multicolumn{1}{|c|}{$\begin{array}{c}\text { Correlations between } \\
\text { simultaneous data (parameter, } \\
\text { time, patient group })\end{array}$} & $\begin{array}{c}\text { Surface } \\
\text { lactoferrin-bearing } \\
\text { granulocytes }(\mathrm{r})\end{array}$ & $\begin{array}{c}\text { Plasma lactoferrin } \\
(\mathrm{r})\end{array}$ & Il-6 (r) \\
\hline ACTH - pre-PCI - SAP & $-0.283(\mathrm{p}=0.213)$ & $-0.158(\mathrm{p}=0.493)$ & $-0.483^{*}(\mathrm{p}=0.042)$ \\
ACTH - pre-PCI - ACS & $0.364(\mathrm{p}=0.115)$ & $0.398(\mathrm{p}=0.082)$ & $-0.298(\mathrm{p}=0.215)$ \\
Cortisol - pre-PCI - SAP & $0.051(\mathrm{p}=0.827)$ & $0.103(\mathrm{p}=0.658)$ & $-0.218(\mathrm{p}=0.385)$ \\
Cortisol - pre-PCI - ACS & $0.391(\mathrm{p}=0.088)$ & $0.499^{*}(\mathrm{p}=0.025)$ & $-0.135(\mathrm{p}=0.581)$ \\
\hline ACTH - post-PCI - SAP & $-0.318(\mathrm{p}=0.160)$ & $-0.136(\mathrm{p}=0.557)$ & $-0.487 *(\mathrm{p}=0.040)$ \\
ACTH - post-PCI - ACS & $-0.044(\mathrm{p}=0.855)$ & $0.051(\mathrm{p}=0.830)$ & $-0.503^{*}(\mathrm{p}=0.034)$ \\
Cortisol - post-PCI - SAP & $-0.218(\mathrm{p}=0.342)$ & $0.017(\mathrm{p}=0.942)$ & $0.195(\mathrm{p}=0.438)$ \\
Cortisol - post-PCI - ACS & $0.083(\mathrm{p}=0.729)$ & $0.217(\mathrm{p}=0.359)$ & $-0.329(\mathrm{p}=0.182)$ \\
\hline ACTH- 1d-PCI - SAP & $-0.601 *(\mathrm{p}=0.004)$ & $-0.435^{*}(\mathrm{p}=0.049)$ & $0.024(\mathrm{p}=0.926)$ \\
ACTH - 1d-PCI - ACS & $-0.541^{*}(\mathrm{p}=0.017)$ & $-0.609^{*}(\mathrm{p}=0.004)$ & $-0.068(\mathrm{p}=0.783)$ \\
Cortisol - 1d-PCI - SAP & $-0.192(\mathrm{p}=0.404)$ & $-0.015(\mathrm{p}=0.950)$ & $0.269(\mathrm{p}=0.280)$ \\
Cortisol - 1d-PCI - ACS & $0.281(\mathrm{p}=0.244)$ & $0.250(\mathrm{p}=0.289)$ & $0.254(\mathrm{p}=0.293)$ \\
\hline
\end{tabular}

SAP: stable angina pectoris, ACS: acute coronary syndrome patients; pre-PCI: directly before, postPCI: directly after, and 1d-PCI: on the following day of PCI (stenting).

Asterisks $(*)$ denote significancy of correlations $(\mathrm{p}<0.05)$.

SAP: $n=21$, ACS n=20; exceptions: IL-6 (ACS post-PCI: n=18, ACS pre- and 1-d-PCI: $n=19$ ) and percentage of lactoferrin-bearing cells (in ACS patients 1-day after PCI: n=19).

6.4.2. Correlations between the MLR and psychosocial factors, neuroendocrine and inflammatory parameters in study II.

Bivariate analysis. Patients who had higher MLR values, had significantly higher levels of plasma CgA, LL-37, IL-6 and CRP (all p-values <0.03) with mostly large effect sizes (Table $8)$.

Multivariate analysis. After adjusting for age, sex, and BMI (and proton pump inhibitor intake and NYHA grade for CgA models, as well), the effect sizes and significance of the correlations between the MLR and CgA, LL-37 improved greatly ( $r>0.6, p=0.003$, 
power>0.95). In contrast, these statistical values for IL-6 and CRP decreased or did not change substantially $(r>0.45, \mathrm{p}<0.04$, power $>0.65)$. Interestingly, the MLR associated with greater depressive symptom severity when further adjustment for sedative intake was employed in the model ( $r>0.6, p=0.004$, power $>0.95)$. As in the bivariate analysis, plasma cortisol and the two selected coping subscales of BSCI (social support, and purpose and connection) did not correlate significantly with the MLR. The significant multivariate-adjusted correlations between the MLR and PNI markers are shown in Fig. 12.

Table 8. Correlations between the MLR and psycho-neuroendocrino-inflammatory system markers in stable CAD patients $(n=23)$

\begin{tabular}{|l|c|c|c|c|c|c|}
\cline { 2 - 7 } \multicolumn{1}{c|}{} & \multicolumn{3}{c|}{ Bivariate correlations } & \multicolumn{3}{c|}{ Multivariate correlations } \\
\cline { 2 - 7 } \multicolumn{1}{c|}{} & $\mathrm{r}$ & $\mathrm{p}$ & power & $\mathrm{r}$ & $\mathrm{p}$ & power \\
\hline Cortisol & 0.279 & n.s. & 0.254 & 0.277 & n.s. & 0.251 \\
\hline Chromogranin A & $0.462^{*}$ & 0.026 & 0.664 & $0.660^{*}$ & 0.003 & 0.980 \\
\hline LL-37 & $0.537^{*}$ & 0.010 & 0.811 & $0.643^{*}$ & 0.003 & 0.963 \\
\hline IL-6 & $0.620^{*}$ & 0.002 & 0.951 & $0.532^{*}$ & 0.016 & 0.819 \\
\hline CRP & $0.474 *$ & 0.022 & 0.692 & $0.474^{*}$ & 0.036 & 0.683 \\
\hline BDI & 0.216 & n.s. & 0.173 & $0.624 *$ & 0.004 & 0.954 \\
\hline BSCI social support & 0.221 & n.s. & 0.180 & -0.260 & n.s. & 0.234 \\
\hline BSCI life meaning & -0.124 & n.s. & 0.088 & -0.084 & n.s. & 0.067 \\
\hline
\end{tabular}

r: Pearson's correlation coefficient, BDI: Beck Depression Inventory, BSCI: Rahe's Brief Stress and Coping Inventory, n.s.: not significant.

Asterisks $(*)$ denote significancy of correlations $(\mathrm{p}<0.05)$.

Exceptions for sample number: $\mathrm{n}=22$ for cortisol and LL-37.

In the multivariate analysis, all parameters were adjusted for age, BMI and sex; additional adjustments for chromogranin A: intake of proton pump inhibitors and grades of heart failure; for BDI: intake of sedatives. 

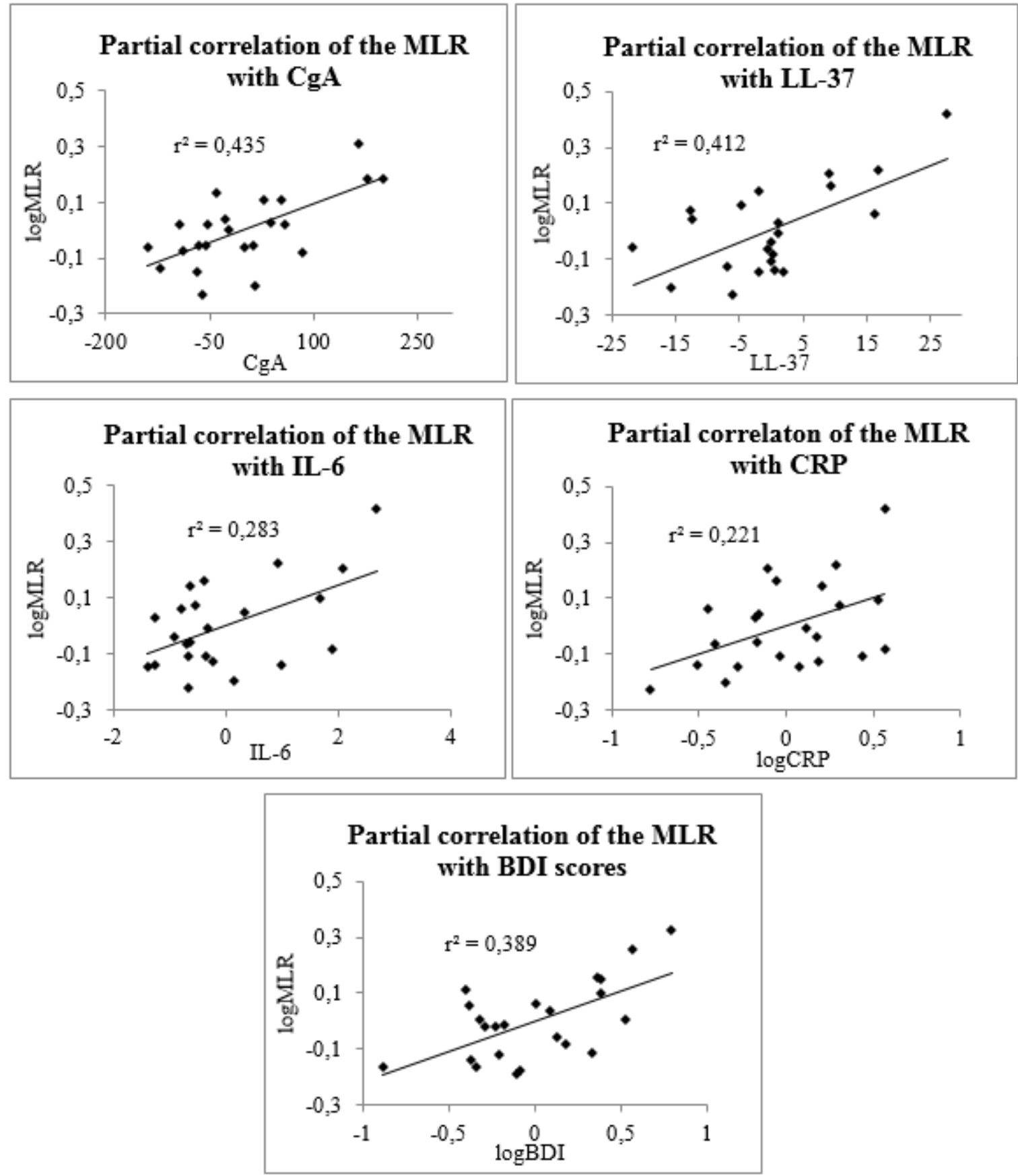

Figure 12. Multivariate-adjusted correlation plots of the MLR and PNI system markers in stable CAD patients $(n=23)$

$r^{2}$ : square of the (Pearson) correlation coefficient, MLR: monocyte-to-lymphocyte ratio, CgA: chromogranin A, BDI: Beck Depression Inventory; exception for sample number: $\mathrm{n}=22$ for LL-37. 
6.4.3. Correlations amongst psychosocial factors, neuroendocrine and inflammatory factors in study II.

Both in the bivariate and multivariate model, we found significant and large effects for the association between lower social support scores and higher $\mathrm{CgA}$ plasma levels and between lower meaning in life and higher CRP plasma values $(r<-0.45$, all $p$-values $<0.04$, power $>0.65$ ), as can be seen in Table 9. Additionally, more severe depressive symptoms associated with higher LL-37 plasma concentrations $(r>0.5, \mathrm{p}<0.04$, power $>0.7)$ and especially strongly with elevated plasma $\mathrm{CgA}$ levels in the adjusted multivariate model $(\mathrm{r}>0.6, \mathrm{p}<0.01$, power $>0.9)$. The significant multivariate-adjusted correlations among psychosocial factors, neuroendocrine and inflammatory markers are shown in Fig. 13.

Table 9. Correlations amongst psychological/psychosocial factors and neuroendocrine and inflammatory markers in stable CAD patients $(\mathrm{n}=23)$

\begin{tabular}{|l|c|c|c|c|c|c|}
\cline { 2 - 7 } \multicolumn{1}{c|}{} & \multicolumn{3}{c|}{ Bivariate correlations } & \multicolumn{3}{c|}{ Multivariate correlations } \\
\cline { 2 - 7 } \multicolumn{1}{c|}{} & $\mathrm{r}$ & $\mathrm{p}$ & power & $\mathrm{r}$ & $\mathrm{p}$ & power \\
\hline BDI - CgA & 0.275 & n.s. & 0.258 & $0.618^{*}$ & 0.008 & 0.949 \\
\hline BDI - LL-37 & 0.380 & n.s. & 0.45 & $0.502^{*}$ & 0.034 & 0.736 \\
\hline BSCI social support - CgA & $-0.502 *$ & 0.015 & 0.756 & $-0.511^{*}$ & 0.030 & 0.776 \\
\hline BSCI life meaning - CRP & $-0.555^{*}$ & 0.006 & 0.862 & $-0.467 *$ & 0.038 & 0.676 \\
\hline
\end{tabular}

r: Pearson's correlation coefficient, BDI: Beck Depression Inventory, BSCI: Rahe's Brief Stress and Coping Inventory, $\mathrm{CgA}$ : chromogranin A, n.s.: not significant. (Exception for sample number: $\mathrm{n}=22$ for LL-37.)

Asterisks $(*)$ denote significancy of correlations $(\mathrm{p}<0.05)$.

In the multivariate analysis, all parameters were adjusted for age, BMI and sex; additional adjustments for $\mathrm{CgA}$ : intake of proton pump inhibitors, grades of heart failure and for BDI: intake of sedatives. 

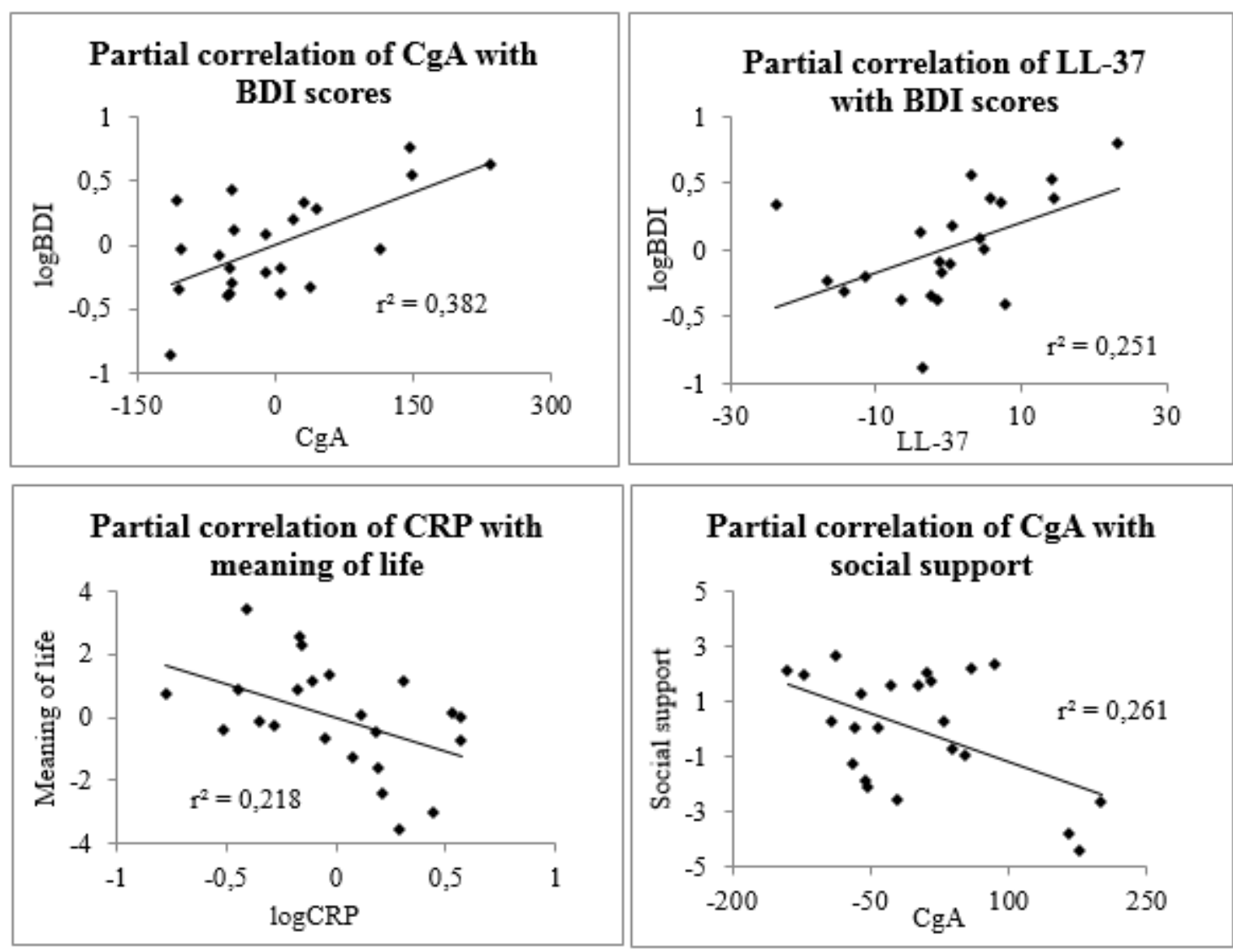

Figure 13. Multivariate-adjusted correlation plots of psychological/psychosocial factors and neuroendocrine and inflammatory markers in stable CAD patients $(n=23)$

$\mathrm{r}^{2}$ : square of the (Pearson) correlation coefficient, CgA: chromogranin A, BDI: Beck Depression Inventory; exception for sample number: $\mathrm{n}=22$ for LL-37.

\section{Discussion}

Our main findings from study I. suggest that pre- and post-PCI states in both SAP and ACS patients may be associated with an increased ratio of activated/degranulated neutrophils expressing lactoferrin on their surface and releasing it. ACS patients were highly stressed and had an overactivated HPA axis; their plasma lactoferrin correlated with plasma cortisol before PCI. On the following day after PCI, both plasma lactoferrin and the ratio of surface lactoferrinbearing granulocytes decreased significantly in both groups, this could indicate declined granulocyte activation that may be due to the negative immune-modulatory effect of ACTH as suggested by negative correlations. 
Our main findings from study II. is that increased MLR could be associated not only with inflammatory factors implicated in atherosclerosis, but with depressive symptoms and with elevated neuroendocrino-sympathetic activity (marked by increased plasma $\mathrm{CgA}$ ) as well in stable CAD. Additionally, enhanced neuroendocrino-sympathetic activity was found to be associated with elevated depressive symptom level and with decreased social support.

\subsection{Stress and inflammatory parameters in CAD patients undergoing stenting}

The highly elevated plasma cortisol level of ACS patients indicated that they were in an extremely stressed state before PCI; the substantially reduced plasma cortisol values on the day after PCI reflects a more relaxed state. Plasma cortisol of SAP patients remained mostly unreactive and did not change noticeably that may be due to a chronically overloaded, exhausted HPA axis [115]. The final low ACTH value of the ACS patients suggests that the initially overactivated HPA axis returned to a much more decreased, normal functioning state after stenting. The moderately decreased 1d-PCI plasma ACTH concentration of SAP patients could indicate a relief after stenting. The statistically significant alterations in plasma cortisol and ACTH values could not be due to the diurnal fluctuation, as the peaks of their concentration in blood are in the early morning (before 6 a.m.).

Leukocytes constitutively express L-selectin in their plasma membrane. In case of leukocyte activation, it is being cleaved from the cell surface, as can be seen in patients with unstable angina [96]. However, its increased cell surface presence may also accompany cell activation, e.g. after a mechanical trauma [116]. The ratio of L-selectin-bearing granulocytes in SAP patients increased slightly during PCI, then it decreased moderately, although these differences were not substantial, they were found to be statistically significant. These changes may reflect homeostatic alterations in these activated granulocytes (shedding balanced by reappearance).

CD15 (Lewis-X) is a tetrasaccharide ligand to selectins on the surface of all myeloid cells; neutrophils can mobilize it from their azurophilic/primary granules upon stimulation [52]. The ratio of CD15-carrying cells increased slightly directly after PCI (post-PCI samples of both groups). This statistically significant, though not considerable elevation may reflect modest late granulocyte activation (affecting primary granules) during stenting, independently of group. 
The slight decrease on the following day suggests a less activated state, as in anxious patients in a relatively relaxed condition [76].

Lactoferrin is a specific activation marker of neutrophils: following cell activation and release form secondary granules, it can bound to cell surface and gain access to the circulation [46-48]. Similarly to the changes in the ratio of CD15-bearing cells, the proportion of lactoferrin-carrying neutrophils increased mildly directly after PCI. In parallel, plasma lactoferrin elevated slightly in post-PCI samples, which indicates slight neutrophil activation. One day after the intervention, marked reductions in cell surface and plasma lactoferrin values were observed in both patient groups that indicates a less activated state of neutrophils. Gach et al. (2005) observed a rapid rise of plasma lactoferrin in coronary artery blood directly after stenting in unstable angina patients that returned to its initial value in 6 hours [96]. Our pre-PCI values of surface lactoferrin-carrying neutrophils were also considerably high (>25\%), showing an elevated ratio of activated neutrophils in CAD. Our findings on the increased plasma lactoferrin levels in pre-PCI samples are consistent with those of Videm et al. (2007), who suggested elevated plasma lactoferrin as a great marker for significant atherosclerotic coronary stenosis and showed that it is more reliable, than plasma myeloperoxidase, another neutrophil activation marker [45]. Furthermore, elevated baseline plasma lactoferrin may predict longterm risk for fatal CAD in newly diagnosed diabetes patients that could be the consequence of the primed state of neutrophils, as suggested by Vengen et al. (2010) [117]. We found that plasma lactoferrin and the percentage of surface lactoferrin-bearing cells changed markedly and in a similar way in our patient groups; thus, both might be used as 'neutrophil activity sensors' in CAD patients. However, from a practical point of view, determining lactoferrin from plasma samples seems to be more suitable. (Blood plasma tests are less expensive and less complicated, and they could be probably automated.)

We found that plasma IL-6 increased during the intervention and enhanced especially on the following day of PCI; this could be a consequence of the onset of acute phase reaction. Similarly to our results, Gach et al. (2005) observed an increase of IL-6 during 6-12 hours after PCI in unstable angina patients in parallel with a decline of plasma lactoferrin and myeloperoxidase [96]. Furthermore, Caixeta et al. (2007) detected a significant, but moderate elevation of plasma IL-6 at 6 hours after PCI both in stable and unstable angina patients that was followed by a decrease; however, their study might have missed the peak at $24 \mathrm{~h}-$ as 
suggested [97]. Circulating level of IL-6 has also a strong link with psychological stress in treated HIV-infected patients [118].

\subsection{ACTH- and cortisol-associated neutrophil modulation in CAD patients undergoing stenting}

One of our main findings is that plasma ACTH and/or cortisol could be associated with the activation state of neutrophils in CAD. We showed inverse correlations between ACTH and surface lactoferrin-bearing granulocytes and plasma lactoferrin the following day of PCI in both groups. The supposition that ACTH could be capable to suppress neutrophil activation/degranulation is consistent with the concept of tonal inhibition of immune/inflammatory responsiveness by ACTH [119].

Cortisol and its analogues are well-known anti-inflammatory agents and immune suppressors, therefore, correlation of cortisol with plasma lactoferrin before stenting in ACS appears to be controversial. Still, glucocorticoids may be capable of activating human neutrophils in vitro; and owing to the high ratio of $\alpha$ to $\beta$ type glucocorticoid receptors on them, neutrophils are less susceptible to apoptosis induction by these hormones [73,120]. Moreover, morning plasma cortisol levels were found to be associated with coronary atherosclerosis severity in US Air Force aircrew members and in patients with suspected CAD; thus, cortisol may be proposed as a proatherogenic stress factor [70,71]. Evidence is accumulating that selective inhibition of a cortisol-synthetizing enzyme (11ß-hydroxysteroid dehydrogenase, $11 \beta$-HSD1) may be useful in prevention of atherosclerosis [121,122]. Surprisingly, 11 $\beta$-HSD1 inhibition contributed to substantial prevention of aortic cholesterol accumulation in apoEdeficient mice on high-fat diet [123]. On the other hand, cholesterol feeding for a month led to high cortisol values and to early signs of atherogenesis in rabbits, suggesting that vascular inflammation related to dyslipidemia may stimulate the HPA axis [124]. 


\subsection{The monocyte-to-lymphocyte ratio: a PNI correlate in stable CAD}

The elevated monocyte fraction and increased MLR reflects an inflammatory state in our stable CAD patients; this is supported by the correlations found between the MLR and plasma LL-37, IL-6 and CRP. Interestingly, the reciprocal of the MLR value (1/0.32=3.125) of our patients is almost equal to the critical lymphocyte-to-monocyte ratio of 3.1, characteristic for patients with a high risk of severe (atherosclerotic) limb ischemia, which is often accompanied by CAD [57].

The elevated circulating levels of chromogranin A ( CgA) may suggest a general neuroendocrine overactivity with increased sympathoadrenal activity [80,81]. It should be noted, that we controlled our CgA models for the effect of proton pump inhibitors and myocardial release of CgA (in cardiac pressure/volume overload). Recently, Dutta et al. (2012) showed in apoE-deficient mice that elevated sympathetic activity is directly related to monocytosis through the enhanced release of hematopoietic cells from the bone marrow (mediated by norepinephrine and $\beta 3$-adrenergic receptors) that was followed by increased monocytopoiesis in the spleen [125]. Monocyte release into blood stream could lead to the progression of atherosclerosis via promoting inflammation especially following myocardial infarction [125,126]. Accordingly, increased plasma CgA could reflect sympathetic activation that can lead to monocytosis in blood. This may be in the background of the highly significant correlation between CgA and the MLR. Furthermore, activated monocytes and macrophages are also capable to produce chromogranins [127].

About one-third of the participants had clinically relevant levels of depressive symptoms, and the average global stress-coping score was in the 'sufficient' range only, suggesting limited coping skills under stress amongst our patients. Patients with more severe depressive symptoms had higher plasma $\mathrm{CgA}$ levels, this could be explained by the increased activity of the neuroendocrino-sympathoadrenal system that accompanies depressive symptomatology. Previous studies found that depressed and chronically stressed subjects show sympathoadrenal hyperactivity that may lead to monocyte/macrophage activation via $\beta$ adrenergic receptors in $\mathrm{CAD}[8,128]$.

Proinflammatory cytokines (particularly IL-1) released by activated monocytes/macrophages may be involved in the background of the association between the MLR and depressive symptoms. These cytokines could contribute to the development of 
depressive mood via interfering with neurotransmitter functioning [129,130]. Correlations of the MLR with IL-6 and CRP probably reflect the activated state of monocytes and their secretion of IL-6, the most potent inducer of CRP synthesis and acute phase response in general [90]. IL-6 and CRP are both major factors that are proposed as links between stress/depression and CAD [60,90,128]. According to Nikkheslat et al. (2015), depressed CAD patients could exhibit considerably higher plasma CRP and lower cortisol levels compared to CAD patients without depression [131]. Similarly, considerably high CRP and relatively low plasma cortisol levels were found in our patients that could be a result of long-term negative feedback in chronic stress $[131,132]$.

The association observed between the MLR and LL-37 is not surprising, as monocytes and derived macrophages are known to release LL-37 in atherosclerotic lesions [98,101]. Recently, LL-37 has been proposed as a novel inflammatory marker; it is expressed by various leukocytes and has various antimicrobial and immunomodulatory effects, and it also takes part in apoptosis and wound healing [99]. Interestingly, LL-37 correlated significantly with depressive symptoms in our CAD patients; this supports the concept that a possible connection could exist between inflammation and depression [93].

Association of low social support with elevated CgA suggests that limited emotional/social support may lead to increased psychosocial stress and to elevated sympathoadrenal activity [133]. Based on mostly rodent investigations, Friedler et al. (2015) suggest that elevated sympathetic activity accompanying chronic psychosocial stress (e.g. social isolation) could drive hematopoiesis towards granulocytic/monocytic progenitor cell formation in bone marrow via $\beta$-adrenergic receptor-mediated signalling and altered geneexpression [134]. This notion is in agreement with the mechanism of monocytosis mentioned above $[125,126]$. 


\subsection{Principal findings}

One of our principal findings is that alteration in neutrophil activation state could be related to plasma ACTH and cortisol effects in CAD. In acute CAD patients, pre-PCI cortisol was elevated and correlated with plasma lactoferrin; the decreased 1d-PCI neutrophil activation markers, however, could reflect the negative immunomodulatory effect of ACTH. As high ratio of activated neutrophils can be considered as a risk factor for the initiation and progression of atherosclerosis [35], stress-related regulation of their activity bears considerable clinical importance.

Another major finding is related to the PNI state of patients with stable CAD: we found that elevated MLR correlated with depressive symptoms, with raised sympathetic activity and with an inflammatory state that could be involved in the initiation and/or progression of CAD. Additionally, we found correlations between increased neuroendocrino-sympathetic activity and low social support and depressive symptom severity that could probably reflect the importance of the psychosocial state in stable CAD. Our present findings support some published results on the significance of sympathetic activation in monocytosis and in monocyte activation, and on the importance of psychological risk factors (e.g. limited social support) in CAD by promoting stress $[8,125,126,128,133,135]$.

By integrating the above mentioned results and ideas, a simplified picture of a vicious cycle/network appears: stressed state before PCI or low social support could lead to raised HPA activity and predominant sympathetic activity, which then may facilitate neutrophil activation and could also lead to the elevation of the monocyte count and activity. Proinflammatory cytokines secreted by these activated neutrophils and monocytes facilitate the development of $\mathrm{CAD}$ and/or depression; with the development of CAD, the activity of the PNI network may be further elevated (e.g. through chest pain, stress and anxiety in angina pectoris).

Therefore, our findings appear to underline the importance of stress management programs and psychosocial interventions, which are capable to reduce chronic stress, to boost social support and stress-coping skills and to alleviate depressive symptoms. These programs/interventions could favourably influence the vicious PNI cycle of interacting psychosocial factors, neuroendocrine and inflammatory markers, and thereby, they could effectively improve both the mental and physical health of the CAD patients $[133,136]$. 


\subsection{Limitations}

Our research has a number of limitations.

In study I., the exact background mechanisms of plasma ACTH and cortisol correlations with the inflammatory (neutrophil activation) state could not be fully clarified. Furthermore, some patient characteristics (e.g. life style factors, age, sex, pre-existent illnesses) may confound the presented results. It is known that metabolic syndrome could not only influence plasma ACTH values but it also frequently accompanies CAD [137,138]. However, our patient groups functioned as auto-controlled statistical units (repeated measurements within group, over-time comparisons), thus, these confounding factors had presumably only minor influence. Sample sizes of the groups were relatively small, however, the high levels of significances strengthen the validity of our findings.

In study II., the main limitations were the small sample size and its cross-sectional design. Still, large effect sizes of the statistically significant correlations support the validity of our results. Because of the small sample size, it was not possible to control for additional confounders, like established cardiovascular risk factors, smoking, diabetes, and medications (with the exceptions of proton pump inhibitors and sedatives). Average depressive symptom score remained in the normal range in the majority (70\%) of participants; thus, it stays unclear whether our findings would hold true in a more severely depressed CAD patient sample or not. Because of the lack of a control group, the possibility that similar associations exist amongst the MLR, psychological and psychosocial factors, neuroendocrine and inflammatory markers in healthy subjects cannot be excluded. However, the increased monocyte fraction and the elevated MLR value, the overactivated neuroendocrino-sympathetic system (indicated by elevated plasma $\mathrm{CgA}$ ), and the relatively low social support provide pathological characteristics of our stable CAD patients. 


\section{Conclusion}

Despite the discussed limitations, our first study led to interesting insights into the dynamically changing neutrophil behaviour in CAD patients undergoing PCI. We showed that neutrophils could be activated already before stenting, and till the next day of PCI, their activation state could decrease well below the initial values in both CAD groups. To our best knowledge, we are the first to reveal correlations between plasma ACTH, cortisol and neutrophil activation/degranulation state in CAD patients in connection with stenting. Additionally, we found that plasma lactoferrin could be a sensitive and valuable marker of neutrophil activation that deserves comprehensive methodological studies to evaluate it as a CAD risk marker. Furthermore, our second study revealed that the MLR, an easily available and inexpensive laboratory parameter, may be applied as a PNI correlate marker in the routine clinical monitoring of stable CAD patients. Further investigations are needed in this multidisciplinary field to clarify the detailed functional connections of the PNI network in CAD in order to facilitate the introduction and integration of innovative and more efficient approaches into prevention and treatment of atherosclerosis in the future. 


\section{Acknowledgements}

I am thankful to Professor László Dux, who gave me the opportunity to work as a Ph.D. student at the Department of Biochemistry.

I greatly appreciate the help of my supervisor, Dr. Margit Keresztes for her guidance and her continuous encouragement over the years that helped me to believe in myself and to overcome the frustrations of the unsuccessful publishing.

I am grateful to Professor Tamás Forster, the head of the 2nd Department of International Medicine and Cardiology Centre, who allowed for us to carry out our investigations on the Centre's patients.

I appreciate the support of the cardiologists of our Stress Research Group, Dr. Imre Ungi (the head of the Invasive Cardiology Unit) and especially the help of Dr. Tamás Horváth, who provided the cardiological background for our studies and took part in the recruitment of the study patients.

I am grateful to Beatrix Rafael, the psychologist of our workgroup, for her invaluable help in evaluating psychological tests of the patients.

I am thankful to Dr. Imre Földesi (the head of the Department of Laboratory Medicine) and to Dr. János Gardi (the head of the Endocrine Laboratory at the 1st Department of International Medicine), who helped us with the assaying of the neuroendocrine parameters.

I am grateful to Imre Ocsovszki for his great help in the analysis of cell-surface granulocyte markers using flow cytometry.

I am obliged to Roland von Känel (Department of Neurology, Bern University Hospital, and Department of Psychosomatic Medicine, Clinic Barmelweid, Barmelweid, Switzerland) for professionally editing our manuscript of study II.

I am grateful for the devoted laboratory work of our laboratory technician, Zsuzsa Lajtos.

I am thankful for the indispensable clinical help of Krisztina Hepp, the chief nurse at Invasive Cardiology Unit.

And last but not least, I am grateful to my family, who supported me throughout the years of my work.

This work was supported financially by the 3-year doctoral fellowship of the Hungarian Ministry of Education. 


\section{References}

1 Mack M, Gopal A (2014) Epidemiology, traditional and novel risk factors in coronary artery disease. Cardiol Clin 32:323-332. doi:10.1016/j.ccl.2014.04.003.

2 Nichols M, Townsend N, Scarborough P, Rayner M (2014) Cardiovascular disease in Europe 2014: epidemiological update. Eur Heart J 35:2950-2959. doi:10.1093/eurheartj/ehu299.

3 Mendis S, Puska P, Norrving B (eds) (2011) Global Atlas on Cardiovascular Disease Prevention and Control. WHO, Geneva.

4 Yusuf S, Hawken S, Ounpuu S, Dans T, Avezum A, et al.; INTERHEART Study Investigators (2004) Effect of potentially modifiable risk factors associated with myocardial infarction in 52 countries (the INTERHEART study): case-control study. Lancet 364:937-952. doi:10.1016/S0140-6736(04)17018-9.

5 Ford ES, Capewell S (2011) Proportion of the decline in cardiovascular mortality disease due to prevention versus treatment: public health versus clinical care. Annu Rev Public Health 32:5-22. doi:10.1146/annurev-publhealth-031210-101211.

6 Stenestrand U, Lindbäck J, Wallentin L; RIKS-HIA Registry (2006) Long-term outcome of primary percutaneous coronary intervention vs prehospital and in-hospital thrombolysis for patients with ST-elevation myocardial infarction. JAMA 296:1749-1756. doi:10.1001/jama.296.14.1749.

7 Cannon B (2013) Cardiovascular disease: Biochemistry to behaviour. Nature 493:S2-3. doi:10.1038/493S2a.

8 Rosengren A, Hawken S, Ounpuu S, Sliwa K, Zubaid M, et al. (2004) Association of psychosocial risk factors with risk of acute myocardial infarction in 11119 cases and 13 648 controls from 52 countries (the INTERHEART study). Lancet 364:953-962. doi:10.1016/S0140-6736(04)17019-0.

9 Tobin KJ (2010) Stable angina pectoris: what does the current clinical evidence tell us? J Am Osteopath Assoc 110:364-370. 
10 Braunwald E (2012) Unstable angina and non-ST elevation myocardial infarction. Am J Respir Crit Care Med 185:924-932. doi:10.1164/rccm.201109-1745CI.

11 Thygesen K, Alpert JS, Jaffe AS, Simoons ML, Chaitman BR, et al. (2012) Third universal definition of myocardial infarction. Eur Heart J 33:2551-2567. doi:10.1093/eurheartj/ehs184.

12 Balady GJ, Morise AP (2014) Exercise testing. In: Mann DL, Zipes DP, Libby P, Bonow O: Braunwald's Heart Disease: A Textbook of Cardiovascular Medicine, ed 3. Philadelphia, PA, Elsevier Saunders, chapter 13.

13 Strong JP, Malcom GT, McMahan CA, Tracy RE, Newman WP 3rd, et al. (1999) Prevalence and extent of atherosclerosis in adolescents and young adults: implications for prevention from the Pathobiological Determinants of Atherosclerosis in Youth Study. JAMA 281:727-735. doi:10.1001/jama.281.8.727.

14 Klebanoff SJ (2005) Myeloperoxidase: friend and foe. J Leukoc Biol 77:598-625. doi:10.1189/jlb.1204697.

15 Glass CK, Witztum JL (2001) Atherosclerosis: The road ahead. Cell 104:503-516. doi:10.1016/S0092-8674(01)00238-0.

16 Marui N, Offermann MK, Swerlick R, Kunsch C, Rosen CA, et al. (1993) Vascular cell adhesion molecule-1 (VCAM-1) gene transcription and expression are regulated through an antioxidant-sensitive mechanism in human vascular endothelial cells. J Clin Invest 92:1866-1874. doi:10.1172/JCI116778.

17 Lewis GF, Rader DJ (2005) New insights into the regulation of HDL metabolism and reverse cholesterol transport. Circ Res 96:1221-1232. doi:10.1161/01.RES.0000170946.56981.5c.

18 Falk E (2006) Pathogenesis of atherosclerosis. J Am Coll Cardiol 47:C7-C12. doi:10.1016/j.jacc.2005.09.068.

19 Moore KJ, Sheedy FJ, Fisher EA (2013) Macrophages in atherosclerosis: A dynamic balance. Nat Rev Immunol 13:709-721. doi:10.1038/nri3520. 
20 Kragel AH, Reddy SG, Wittes JT, Roberts WC (1989) Morphometric analysis of the composition of atherosclerotic plaques in the four major epicardial coronary arteries in acute myocardial infarction and in sudden coronary death. Circulation 80:1747-1756. doi:10.1161/01.CIR.80.6.1747.

21 Schwartz SM, Virmani R, Rosenfeld ME (2000) The good smooth muscle cells in atherosclerosis. Curr Atheroscler Rep 2:422-429. doi:10.1007/s 11883-000-0081-5.

22 Barger AC, Beeuwkes R 3rd, Lainey LL, Silverman KJ (1984) Hypothesis: vasa vasorum and neovascularization of human coronary arteries. A possible role in the pathophysiology of atherosclerosis. N Engl J Med 310:175-177. doi:10.1056/NEJM198401193100307.

23 Virmani R, Kolodgie FD, Burke AP, Finn AV, Gold HK, et al. (2005) Atherosclerotic plaque progression and vulnerability to rupture: angiogenesis as a source of intraplaque hemorrhage. Arterioscler Thromb Vasc Biol 25:2054-2061. doi:10.1161/01.ATV.0000178991.71605.18.

24 W van Lammeren G, L Moll F, Borst GJ, de Kleijn DP, P M de Vries JP, et al. (2011) Atherosclerotic plaque biomarkers: beyond the horizon of the vulnerable plaque. Curr Cardiol Rev 7:22-27. doi:10.2174/157340311795677680.

25 Vink A, Schoneveld AH, Richard W, de Kleijn DP, Falk E, et al. (2001) Plaque burden, arterial remodeling and plaque vulnerability: determined by systemic factors? J Am Coll Cardiol 38:718-723. doi:10.1016/S0735-1097(01)01444-9.

26 Pletcher MJ, Tice JA, Pignone M, Browner WS (2004) Using the coronary artery calcium score to predict coronary heart disease events: a systematic review and meta-analysis. Arch Intern Med 164:1285-1292. doi:10.1001/archinte.164.12.1285.

27 Beckman JA, Ganz J, Creager MA, Ganz P, Kinlay S (2001) Relationship of clinical presentation and calcification of culprit coronary artery stenosis. Arterioscler Thromb Vasc Biol 21:1618-1622. doi:10.1161/hq0901.095554.

28 Ehara S, Kobayashi Y, Yoshiyama M, Shimada K, Shimada Y, et al. (2004) Spotty calcification typifies the culprit plaque in patients with acute myocardial infarction: an intravascular ultrasound study. Circulation 110:3424-3429. doi:10.1161/01.CIR.0000148131.41425.E9. 
29 Steinl DC, Kaufmann BA (2015) Ultrasound imaging for risk assessment in atherosclerosis. Int J Mol Sci 16:9749-9769. doi:10.3390/ijms16059749.

30 Libby P, Ridker PM, Hansson GK (2011) Progress and challenges in translating the biology of atherosclerosis. Nature 473:317-325. doi:10.1038/nature10146.

31 Baetta R, Corsini A (2010) Role of polymorphonuclear neutrophils in atherosclerosis: Current state and future perspectives. Atherosclerosis 210:1-13. doi:10.1016/j.atherosclerosis.2009.10.028.

32 Drechsler M, Döring Y, Megens RTA, Soehnlein O (2011) Neutrophilic granulocytes promiscuous accelerators of atherosclerosis. Thromb Haemost 106:839-848. doi:10.1160/TH11-07-0501.

33 Soehnlein O (2012) Multiple roles for neutrophils in atherosclerosis. Circ Res 110:875888. doi:10.1161/CIRCRESAHA.111.257535.

34 Weber C, Zernecke A, Libby P (2008) The multifaceted contributions of leukocyte subsets to atherosclerosis: lessons from mouse models. Nature Rev Immunol 8:802-815. doi:10.1038/nri2415.

35 Mazor R, Schurtz-Swirski R, Farah R, Kristal B, Shapiro G, et al. (2008) Primed polymorphonuclear leukocytes constitute a possible link between inflammation and oxidative stress in hyperlipidemic patients. Atherosclerosis 197:937-948. doi:10.1016/j.atherosclerosis.2007.08.014.

36 Quinn K, Henriques M, Parker T, Slutsky AS, Zhang H (2008) Human neutrophil peptides: a novel potential mediator of inflammatory cardiovascular diseases. Am J Physiol Heart Circ Physiol 295:H1817-H1824. doi:10.1152/ajpheart.00472.2008.

37 Adamsson Eryd S, Smith JG, Melander O, Hedblad B, Engström G (2012) Incidence of coronary events and case fatality rate in relation to blood lymphocyte and neutrophil counts. Arterioscler Thromb Vasc Biol 32:533-539. doi:10.1161/ATVBAHA.111.240416.

38 Avanzas P, Arroyo-Espliguero R, Cosin-Sales J, Quiles J, Zouridakis E, et al. (2004) Multiple complex stenoses, high neutrophil count and C-reactive protein levels in patients with chronic stable angina. Atherosclerosis 175:151-157. doi:10.1016/j.atherosclerosis.2004.03.013. 
39 He J, Li J, Wang Y, Hao P, Hua Q (2014) Neutrophil-to-lymphocyte ratio (NLR) predicts mortality and adverse-outcomes after ST-segment elevation myocardial infarction in Chinese people. Int J Clin Exp Pathol 7:4045-4056.

40 Horne BD, Anderson JL, John JM, Weaver A, Bair TL, et al. (2005) Which white blood cell subtypes predict increased cardiovascular risk? J Am Coll Cardiol 45:1638-1643. doi:10.1016/j.jacc.2005.02.054.

41 Huang G, Zhong XN, Zhong B, Chen YQ, Liu ZZ, et al. (2009) Significance of white blood cell count and its subtypes in patients with acute coronary syndrome. Eur J Clin Invest 39:348-358. doi:10.1111/j.1365-2362.2009.02107.x.

42 Papa A, Emdin M, Passino C, Michelassi, C, Battaglia D, et al. (2008) Predictive value of elevated neutrophil-lymphocyte ratio on cardiac mortality in patients with stable coronary artery disease. Clin Chim Acta 395:27-31. doi:10.1016/j.cca.2008.04.019.

43 Buffon A, Biasucci LM, Liuzzo G (2002) Widespread coronary inflammation in unstable angina. New Engl J Med 347:5-12. doi:10.1056/NEJMoa012295.

44 Ott I, Neumann FJ, Gawaz M, Schmitt M, Schömig A (1996) Increased neutrophil-platelet adhesion in patients with unstable angina. Circulation 94:1239-1246. doi:10.1161/01.CIR.94.6.1239.

45 Videm V, Wiseth R, Gunnes S, Madsen HO, Garred P (2007) Multiple inflammatory markers in patients with significant coronary artery disease. Int J Cardiol 118:81-87. doi:10.1016/j.ijcard.2006.07.005.

46 Afeltra A, Caccavo D, Ferri GM, Addessi MA, de Rosa FG, et al. (1997) Expression of lactoferrin on human granulocytes: analysis with polyclonal and monoclonal antibodies. Clin Exp Immunol 109:279-285. doi:10.1046/j.1365-2249.1997.4351333.x.

47 Boxer LA, Haak RA, Yang HH, Wolach JB, Whitcomb JA, et al. (1982) Membrane-bound lactoferrin alters the surface properties of polymorphonuclear leukocytes. J Clin Invest 70:1049-1057. doi:10.1172/JCI110692.

48 Swain SD, Jutila KL, Quinn MT (2000) Cell-surface lactoferrin as a marker for degranulation of specific granules in bovine neutrophils. Am J Vet Res 61:29-37. doi:10.2460/ajvr.2000.61.29. 
49 Legrand D, Elass E, Carpentier M, Mazurier J (2005) Lactoferrin: a modulator of immune and inflammatory responses. Cell Mol Life Sci 62:2549-2559. doi:10.1007/s00018-005$5370-2$.

50 Kansas GS (1992) Structure and function of L-selectin. APMIS 100:287-293. doi:10.1111/j.1699-0463.1992.tb00874.x.

51 Nakayama F, Nishihara S, Iwasaki H, Kudo T, Okubo R, et al. (2001) CD15 expression in mature granulocytes is determined by alpha 1,3-fucosyltransferase IX, but in promyelocytes and monocytes by alpha 1,3-fucosyltransferase IV. J Biol Chem 276:1610016106. doi:10.1074/jbc.M007272200.

52 Suzuki H, Yokomizo S, Wakamoto S, Watanabe K, Hirose K, et al. (2000) Translocation of sLe(x) on the azurophilic granule membrane to the plasma membrane in activated human $\begin{array}{lllll}\text { neutrophils. } & \mathrm{J} & \text { Electron } & \text { Microsc } & \text { (Tokyo) }\end{array}$ doi:10.1093/oxfordjournals.jmicro.a023816.

53 Plutzky J (2001) Inflammatory pathways in atherosclerosis and acute coronary syndromes. Am J Cardiol 88:10K-15K. doi:10.1016/S0002-9149(01)01924-5.

54 Matsuura E, Kobayashi K, Matsunami Y, Shen L, Quan N, et al. (2009) Autoimmunity, infectious immunity, and atherosclerosis. J Clin Immunol 29:714-721. doi:10.1007/s10875-009-9333-5.

55 Nozawa N, Hibi K, Endo M, Sugano T, Ebina T, et al. (2010) Association between circulating monocytes and coronary plaque progression in patients with acute myocardial infarction. Circ J 74:1384-1391. doi:10.1253/circj.CJ-09-0779.

56 Waterhouse DF, Cahill RA, Sheehan F, McCreery CJ (2008) Prediction of calculated future cardiovascular disease by monocyte count in asymptomatic population. Vasc Health Risk Manag 4:177-187. doi:10.2147/VHRM.S2240.

57 Gary T, Pichler M, Belaj K, Eller P, Hafner F, et al. (2014) Lymphocyte-to-monocyte ratio: a novel marker for critical limb ischemia in PAOD patients. Int J Clin Pract 68:1483-1487. doi:10.1111/ijcp.12495. 
58 Núnez J, Sanchis J, Bodí V, Núnez E, Mainar L, et al. (2009) Relationship between low lymphocyte count and major cardiac events in patients with acute chest pain, a nondiagnostic electrocardiogram and normal troponin levels. Atherosclerosis 206:251-257. doi:10.1016/j.atherosclerosis.2009.01.029.

59 Everson-Rose SA, Lewis TT (2005) Psychosocial factors and cardiovascular diseases. Annu Rev Public Health 26:469-500. doi:10.1146/annurev.publhealth.26.021304.144542.

60 Joynt KE, Whellan DJ, O'Connor CM (2003) Depression and cardiovascular disease: Mechanisms of interaction. Biol Psychiatry 54:248-261. doi:10.1016/S00063223(03)00568-7.

61 Lichtman JH, Bigger JT, Blumenthal JA, Frasure-Smith N, Kaufmann PG, et al. (2008) Depression and coronary heart disease (Recommendations for screening, referral, and treatment). Circulation 118:1768-1775. doi:10.1161/CIRCULATIONAHA.108.190769.

62 Steptoe A, Kivimaki M (2013) Stress and cardiovascular disease: an update on current knowledge. Annu Rev Public Health 34:337-354. doi:10.1146/annurev-publhealth031912-114452.

63 Barth J, Schneider S, von Känel R (2010) Lack of social support in the etiology and the prognosis of coronary heart disease: a systematic review and meta-analysis. Psychosom Med 72:229-238. doi:10.1097/PSY.0b013e3181d01611.

64 Skrabski Á, Kopp M, Rózsa S, Réthelyi J, Rahe RH (2005) Life meaning: an important correlate of health in the Hungarian population. Int $\mathrm{J}$ Behav Med 12:78-85. doi:10.1207/s15327558ijbm1202_5.

65 Vedhara K, Irwin M (eds) (2005) Human Psychoneuroimmunology. USA, Oxford University Press.

66 Selye H (1974) Stress and distress. In: George Serban (eds): Psychopathology of Human Adaptation. Springer, pp. 137-146.

67 Chrousos GP (1995) The hypothalamic-pituitary-adrenal axis and immune-mediated inflammation (Seminars of Beth Israel Hospital, Boston). New Engl J Med 332:1351-1362. doi:10.1056/NEJM199505183322008. 
68 Glaser R, Kiecolt-Glaser JK (2005) Stress-induced immune dysfunction: implications for health. Nat Rev Immunol 5:243-251. doi:10.1038/nri1571.

69 Bellinger DL, Lorton D, Lubahn C, Felten DL (2011) In: Ader R, Felten DL, Cohen N (eds): Psychoneuroimmunology ed 3 Vol 2. San Diego, Academic, pp. 55-112.

70 Alevizaki M, Cimponeriu A, Lekakis J (2007) High anticipatory stress plasma cortisol levels and sensitivity to glucocorticoids predict severity of coronary artery disease in subjects undergoing coronary angiography. Metabolism 56:222-226. doi:10.1016/j.metabol.2006.09.017.

71 Troxler RG, Sprague EA, Albanese RA, Fuchs R, Thompson AJ (1977) The association of elevated plasma cortisol and early atherosclerosis as demonstrated by coronary angiography. Atherosclerosis 26:151-162. doi:10.1016/0021-9150(77)90098-3.

72 Hamer M, Endrighi R, Venuraju SM, Lahiri A, Steptoe A (2012) Cortisol responses to mental stress and the progression of coronary artery calcification in healthy men and women. Plos ONE 7:e31356. doi:10.1371/journal.pone.0031356.

73 Strickland I, Kisich K, Hauk PJ, Votterod A, Chrousos GP, et al. (2001) High constitutive glucocorticoid receptor beta in human neutrophils enables them to reduce their spontaneous rate of cell death in response to corticosteroids. J Exp Med 193:585-593. doi:10.1084/jem.193.5.585.

74 Yudkin JS, Kumari M, Humphries SE, Mohamed-Ali V (2000) Inflammation, obesity, stress and coronary heart disease: is interleukin-6 the link? Atherosclerosis. 148:209-214. doi:10.1016/S0021-9150(99)00463-3.

75 Ellard DR, Castle PC, Mian R (2001) The effect of a short-term mental stressor on neutrophil activation. Int J Psychophysiol 41:93-100. doi:10.1016/S0167-8760(00)00180X.

76 Keresztes M, Rudisch T, Tajti J, Ocsovszki I, Gardi J (2007) Granulocyte activation in humans is modulated by psychological stress and relaxation. Stress 10:271-281. doi:10.1080/10253890701248079. 
77 Mian R, Shelton-Rayner G, Harkin B, Williams P (2003) Observing a fictitious stressful event: haematological changes, including circulating leukocyte activation. Stress 6:41-47. doi:10.1080/1025389031000101349.

78 Köllner V, Bernardy K (2006) How to reduce stress and anxiety in patients undergoing catheterization? Clin Res Cardiol 95:511-513. doi:10.1007/s00392-006-0434-3.

79 Gidron Y, Armon T, Gilutz H, Huleihel M (2003) Psychological factors correlate meaningfully with percent-monocytes among acute coronary syndrome patients. Brain Behav Immun 17:310-315. doi:10.1016/S0889-1591(03)00061-8.

80 Cryer PE, Wortsman J, Shah SD, Nowak RM, Deftos LJ (1991) Plasma chromogranin A as a marker of sympathocromaffin activity in humans. Am J Physiol Endocrinol Metab 260:E243-E246.

81 Omland T, Dickstein K, Syversen U (2003) Association between plasma chromogranin A concentration and long-term mortality after myocardial infarction. Am J Med 114:25-30. doi:10.1016/S0002-9343(02)01425-0.

82 Jansson AM, Rosjo H, Omland T, Karlsson T, Hartford M, et al. (2009) Prognostic value of circulating chromogranin A levels in acute coronary syndromes. Eur Heart J 30:25-32. doi:10.1093/eurheartj/ehn513.

83 Kawada S, Fukusaki C, Ohtani M, Kobayashi K (2009) Effects of hyperoxic inhalation on psychological stress-induced salivary biomarkers. Biomed Res 30:245-249. doi:10.2220/biomedres.30.245.

84 Rai B, Kaur J (2011) Salivary stress markers and psychological stress in simulated microgravity: 21 days in $6^{\circ}$ head-down tilt. J Oral Sci 53:103-107. doi:10.2334/josnusd.53.103.

85 Fukui M, Hinode D, Yokoyama M, Yoshioka M, Kataoka K, et al. (2010) Levels of salivary stress markers in patients with anxiety about halitosis. Arch Oral Biol 55:842-847. doi:10.1016/j.archoralbio.2010.07.014.

86 Wagner J, Cik M, Marth E, Santner BI, Gallasch E, et al. (2010) Feasibility of testing three salivary stress biomarkers in relation to naturalistic traffic noise exposure. Int $\mathrm{J}$ Hyg Environ Health 213:153-155. doi:10.1016/j.ijheh.2009.08.004. 
87 Katsuura S, Kamezaki Y, Yamagishi N, Kuwano Y, Nishida K, et al. (2011) Circulating vascular endothelial growth factor is independently and negatively associated with trait anxiety and depressive mood in healthy Japanese university students. Int J Psychophysiol 81:38-43. doi:10.1016/j.ijpsycho.2011.04.004.

88 Tsubouchi H, Nakai Y, Toda M, Morimoto K, Chang YS, et al. (2011) Change of salivary stress marker concentrations during pregnancy: maternal depressive status suppress changes of those levels. J Obstet Gynaecol Res 37:1004-1009. doi:10.1111/j.14470756.2010.01473.x.

89 Libby P, Sukhova G, Lee RT, Galis ZS (1995) Cytokines regulate vascular functions related to stability of the atherosclerotic plaque. J Cardiovasc Pharmacol 25:S9-12.

90 Woods A, Brull DJ, Humphries SE, Montgomery HE (2000) Genetics of inflammation and risk of coronary artery disease: the central role of interleukin-6. Eur Heart J 21:1574-1583. doi:10.1053/euhj.1999.2207.

91 Ramachandran SV (2006) Biomarkers of cardiovascular disease: molecular basis and practical considerations. Circulation 113:2335-2362. doi:10.1161/CIRCULATIONAHA.104.482570.

92 Kiecolt-Glaser JK, Gouin JP, Hantsoo L (2010) Close relationships, inflammation, and health. Neurosci Biobehav Rev 35:33-38. doi:10.1016/j.neubiorev.2009.09.003.

93 Stewart JC, Rand KL, Muldoon MF, Kamarck TW (2009) Aspective evaluation of the directionality of the depression-inflammation relationship. Brain Behav Immun 23:936944. doi:10.1016/j.bbi.2009.04.011.

94 Tecchio C, Micheletti A, Cassatella MA (2014) Neutrophil-Derived Cytokines: Facts Beyond Expression. Front Immunol 5:508. doi:10.3389/fimmu.2014.00508.

95 Murdoch C, Finn A (2000) Chemokine receptors and their role in inflammation and infectious diseases. Blood 95:3032-3043.

96 Gach O, Biémar C, Nys M, Deby-Dupont G, Chapelle JP, et al. (2005) Early release of neutrophil markers of activation after direct stenting in patients with unstable angina. Coron Art Dis 16:59-65. 
97 Caixeta AM, Brito FS, Costa MA, Serrano CV, Petriz JL, et al. (2007) Enhanced inflammatory response to coronary stenting marks the development of clinically relevant restenosis. Catheter Cardiovasc Interv 69:500-507. doi:10.1002/ccd.21007.

98 Edfeldt K, Agerberth B, Rottenberg ME, Gudmundsson GH, Wang XB, et al. (2006) Involvement of the antimicrobial peptide LL-37 in human atherosclerosis. Arterioscler Thromb Vasc Biol, 26:1551-1557. doi:10.1161/01.ATV.0000223901.08459.57.

99 Vandamme D, Landuyt B, Luyten W, Schoofs L (2012) A comprehensive summary of LL37, the factotum human cathelicidin peptide. Cell Immunol 280:22-35. doi:10.1016/j.cellimm.2012.11.009.

100 Agerberth B, Charo J, Werr J, Olsson B, Idali F, et al. (2000) The human antimicrobial and chemotactic peptides LL-37 and alpha-defensins are expressed by specific lymphocyte and monocyte populations. Blood 2000. 96:3086-3093.

101 Ciornei CD, Tapper H, Bjartell A, Sternby NH, Bodelsson M (2006) Human antimicrobial peptide LL-37 is present in atherosclerotic plaques and induces death of vascular smooth muscle cells: a laboratory study. BMC Cardiovasc Disord 6:49. doi:10.1186/1471-2261-649.

102 Koczulla R, von Degenfeld G, Kupatt C, Krötz F, Zahler S, et al. (2003) An angiogenic role for the human peptide antibiotic LL-37/hCAP-18. J Clin Invest 111:1665-1672. doi:10.1172/JCI17545.

103 Kahlenberg JM, Kaplan MJ (2013) Little peptide, big effects: the role of LL-37 in inflammation and autoimmune disease. J Immunol 191:4895-4901. doi:10.4049/jimmunol.1302005.

104 Antonsen S, Wiggers P, Dalhøj J, Blaabjerg O (1993) An enzyme-linked immunosorbent assay for plasma-lactoferrin. Concentrations in 362 healthy, adult blood donors. Scand J Clin Lab Invest 53:133-144. doi:10.3109/00365519309088400.

105 Wilson MB, Nakane PK (1987) Recent development in the periodate method of conjugating horseradish peroxidase. In: Knapp W, Holubar K, Wick G (eds): Immunofluorescence and related staining techniques. Amsterdam, Elsevier, pp. 215-224. 
106 Beck AT, Ward CH, Mendelson M, Mock J, Erbaugh J (1961) An inventory for measuring depression. Arch Gen 4:561-571. doi:10.1001/archpsyc.1961.01710120031004.

107 Ágoston G, Szili I (2001) Diagnostic questionnaires and symptom-rating scales. In: Füredi J, Németh A, Tariska P (eds): The Hungarian handbook of psychiatry. Budapest, Medicina (in Hungarian)

108 Rahe HR, Tolles RL (2002) The brief stress and coping inventory: a useful stress management instrument. Int J Stress Manag 9:61-70. doi:10.1023/A:1014950618756.

109 Rózsa S, Kö N, Csoboth Cs, Purebl Gy, Beöthy-Molnár A, et al. (2005) Stress and coping. Hungarian experiences with Rahe Brief Stress and Coping Inventory. Mentálhigiéné és Pszichoszomatika 6:275-294. (in Hungarian) doi:10.1556/Mental.6.2005.4.2.

110 Cohen J (1988) Statistical power for the behavioral sciences, ed 3. New York, Academic.

111 Perneger TV (1998) What's wrong with Bonferroni adjustments. BMJ 316:1236-1238. doi:10.1136/bmj.316.7139.1236.

112 Feise RJ (2002) Do multiple outcome measures require p-value adjustment? BMC Med Res Methodol 2:8. doi:10.1186/1471-2288-2-8.

113 Glinicki P, Jeske W (2010) Chromogranin A (CgA) - the influence of various factors in vivo and in vitro, and existing disorders on its concentration in blood. Endokrynol Pol 61:384-387.

114 Glinicki P, Jeske W, Kapuścińska R, Zgliczyński W (2015) Comparison of chromogranin $\mathrm{A}(\mathrm{CgA})$ levels in serum and plasma (EDTA2K) and the respective reference ranges in healthy males. Endokrynol Pol 66:53-56. doi:10.5603/EP.2015.0009.

115 Nijm J, Kristenson M, Olsson AG, Jonasson L (2007) Impaired cortisol response to acute stressors in patients with coronary disease. Implications for inflammatory activity. J Int Med 262:375-384. doi:10.1111/j.1365-2796.2007.01817.x.

116 Cocks RA, Chan TYF, Rainer T (1998) Leukocyte L-selectin is up-regulated after mechanical trauma in adults. J Trauma (Injury Infect Crit Care) 45:1-6. 
117 Vengen IT, Dale AC, Wiseth R, Midthjelld K, Videm V (2010) Lactoferrin is a novel predictor of fatal ischemic heart disease in diabetes mellitus type 2: Long-term follow-up of the HUNT 1 study. Atherosclerosis 212:614-620. doi:10.1016/j.atherosclerosis.2010.06.008.

118 Fumaz CR, Gonzalez-Garcia M, Borras X, Munoz-Moreno JA, Perez-Alvarez N, et al. (2012) Psychological stress is associated with high levels of IL-6 in HIV-1 infected individuals on effective combined antiretroviral treatment. Brain Behav Immun 26:568572. doi:10.1016/j.bbi.2012.01.001.

119 Stefano GB, Smith EM (1996) Adrenocorticotropin - a central trigger in immune responsiveness: tonal inhibition of immune activation. Med Hypotheses 46:471-478. doi:10.1016/S0306-9877(96)90028-6.

120 Strausbaugh HJ, Rosen SD (2001) A potential role for annexin 1 as a physiologic mediator of glucocorticoid-induced L-selectin shedding from myeloid cells. J Immunol 166:62946300. doi:10.4049/jimmunol.166.10.6294.

121 Fantidis P (2010) The role of the stress-related anti-inflammatory hormones ACTH and cortisol in atherosclerosis. Curr Vasc Pharmacol 8:517-525. doi:10.2174/157016110791330889.

122 Walker BR (2007) Glucocorticoids and cardiovascular disease. Eur J Endocrinol 157:545559. doi:10.1530/EJE-07-0455.

123 Hermanowski-Vosatka A, Balkovec JM, Cheng K, Chen HY, Hernandez M, et al. (2005) $11 \beta$-HSD1 inhibition ameliorates metabolic syndrome and prevents progression of atherosclerosis in mice. J Exp Med 202:517-527. doi:10.1084/jem.20050119.

124 de Prada TP, Pozzi AO, Coronado MT, Pounchard MA, Gonzalez P, et al. (2007) Atherogenesis takes place in cholesterol-fed rabbits when in cholesterol-fed rabbits when circulating concentrations of endogenous cortisol are increased and inflammation suppressed. Atherosclerosis 191:333-339. doi:10.1016/j.atherosclerosis.2006.05.049.

125 Dutta P, Courties G, Wei Y, Leuschner F, Gorbatov R, et al. (2012) Myocardial infarction accelerates atherosclerosis. Nature 487:325-329. doi:10.1038/nature11260. 
126 Tabas I (2012) Cardiology: Bad matters made worse. Nature 487:306-308. doi:10.1038/487306a.

127 Tasiemski A, Hammad H, Vandenbulcke F, Breton C, Bilfinger TJ, et al. (2002) Presence of chromogranin-derived antimicrobial peptides in plasma during coronary artery bypass surgery and evidence of an immune origin of these peptides. Blood 100:553-559. doi:10.1182/blood.V100.2.553.

128 Black PH, Garbutt LD (2002) Stress, inflammation and cardiovascular disease. J Psychosom Res 52:1-23. doi:10.1016/S0022-3999(01)00302-6.

129 Kronfol Z, Remick DG (2000) Cytokines and the brain: implications for clinical psychiatry. Am J Psychiatry 157:683-694.

130 Miller AH, Haroon E, Raison CL, Felger JC (2013) Cytokine targets in the brain: impact on neurotransmitters and neurocircuits. Depress Anxiety 30:297-306. doi:10.1002/da.22084.

131 Nikkheslat N, Zunszain PA, Horowitz MA, Barbosa IG, Parker JA, et al. (2015) Insufficient glucocorticoid signaling and elevated inflammation in coronary heart disease patients with comorbid depression. Brain Behav Immun 48:8-18. doi:10.1016/j.bbi.2015.02.002.

132 Miller GE, Chen E, Zhou ES (2007) If it goes up, must it go down? Chronic stress and the hypothalamic-pituitary-adrenocortical axis in humans. Psychol Bull 133:25-45. doi:10.1037/0033-2909.133.1.25.

133 Rozanski A, Blumenthal JA, Davidson K, Saab PG, Kubzansky L (2005) The epidemiology, pathophysiology, and management of psychosocial risk factors in cardiac patients. Journal of the American College of Cardiology J Am Coll Cardiol 45:637-651. doi:10.1016/j.jacc.2004.12.005.

134 Friedler B, Crapser J, McCullough L (2015) One is the deadliest number: the detrimental effects of social isolation on cerebrovascular diseases and cognition. Acta Neuropathol 129:493-509. doi:10.1007/s00401-014-1377-9. 
135 Pizzi C, Manzoli L, Mancini S, Bedetti G, Fontana F, et al. (2010) Autonomic nervous system, inflammation and preclinical carotid atherosclerosis in depressed subjects with $\begin{array}{llll}\text { coronary } & \text { risk } & \text { factors. } & \text { Atherosclerosis }\end{array}$ doi:10.1016/j.atherosclerosis.2010.04.038.

136 Zeng W, Stason WB, Fournier S, Razavi M, Ritter G, et al. (2013) Benefits and costs of intensive lifestyle modification programs for symptomatic coronary disease in Medicare beneficiaries. American Heart Journal Am Heart J 165:785-792. doi:10.1016/j.ahj.2013.01.018.

137 Praveen EP, Sahoo JP, Kulshreshtha B, Khurana ML, Gupta N, et al. (2011) Morning cortisol is lower in obese individuals with normal glucose tolerance. Diabetes Metab Syndr Obes 4:347-352. doi:10.2147/DMSO.S23915.

138 Kazakou P, Kyriazopoulou V, Michalaki M, Ierodiakonou V, Psyrogiannis A, et al. (2012) Activated hypothalamic pituitary adrenal axis in patients with metabolic syndrome. Horm Metab Res 44:839-844. doi:10.1055/s-0032-1311632. 
11. Annex

Figure A8

Table A3

Table A4

Table A5

Table A6

I. publication

II. publication 

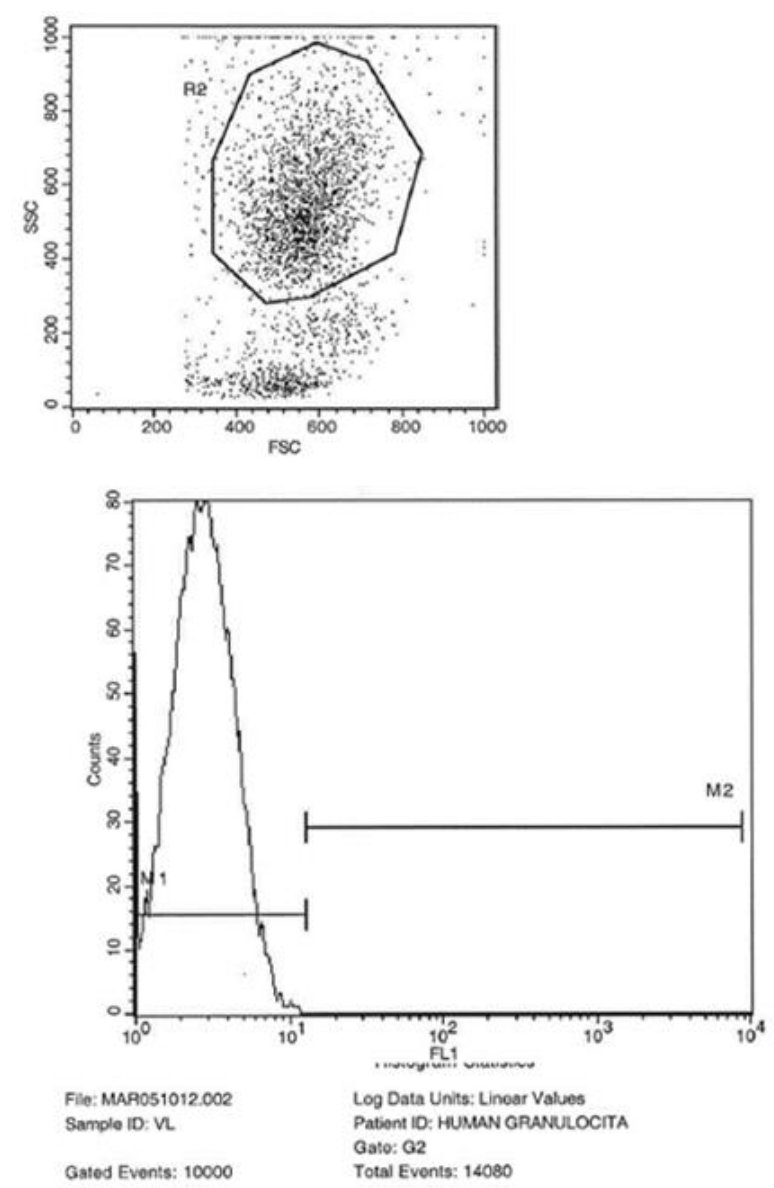

\begin{tabular}{rrrrrrrr} 
Marker Lot, Right Events \% Gated \% Total Moan Geo Moan & CV & Modan Poak Ch \\
\hline All 1. 19910 & 10000 & 100.00 & 71.02 & 3.06 & 2.74 & 69.48 & 2.74 \\
\hline
\end{tabular}

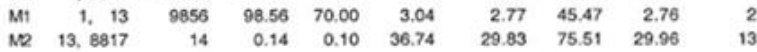

L-selectin control
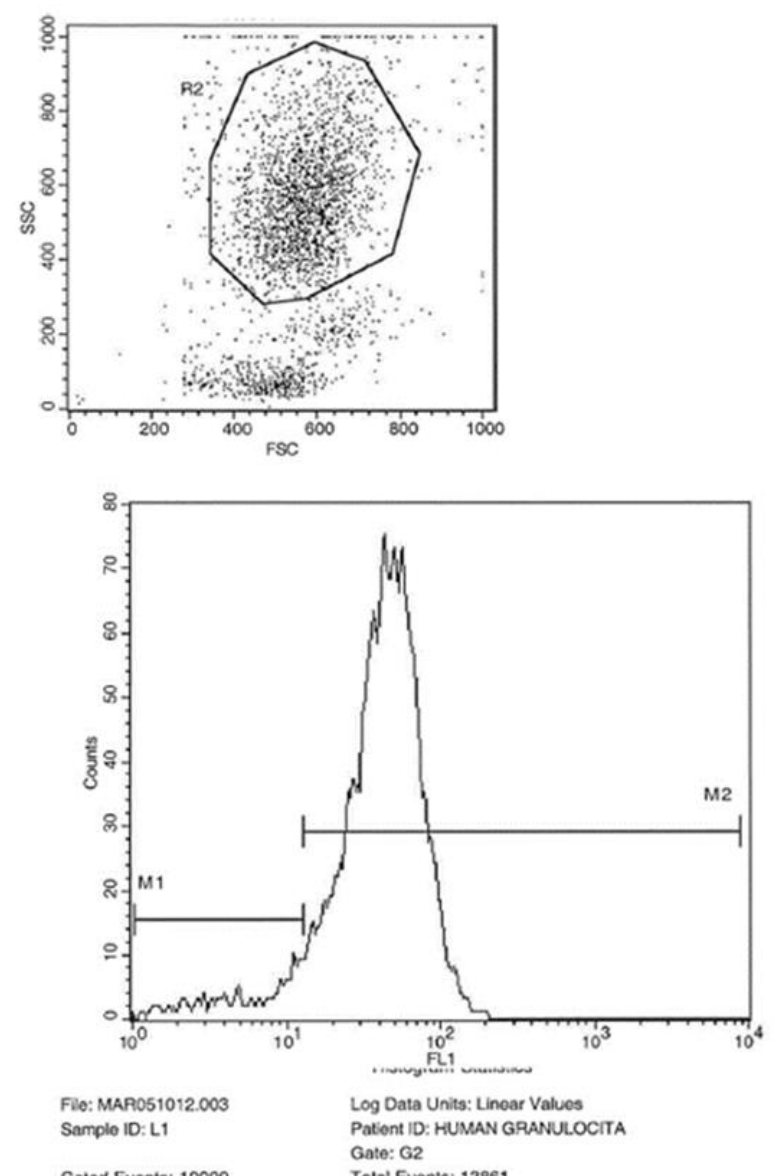

Gated Events: 10000

Markor Loff, Right Events \% Gated \% Total Nean Geo Mean CV Modian PeakCh $\begin{array}{lrrrrrrrrr}\text { M1 } & 1,13 & 766 & 7.66 & 5.53 & 6.81 & 5.61 & 54.29 & 6.61 & 10 \\ \text { N2 } & 13,8817 & 9236 & 92.36 & 66.63 & 48.57 & 43.21 & 49.43 & 44.51 & 55\end{array}$

L-selectin sample (pre-PCI)

Figure A8. Examples for flow cytometry measures: L-selectin control and sample (pre-PCI) results. 
Table A3. Stress hormone levels with statistical analysis in SAP and ACS patients in study I. (mean \pm SD, p-values)

\begin{tabular}{|c|c|c|c|c|c|c|c|}
\hline \multirow[t]{2}{*}{ Parameters } & \multicolumn{4}{|c|}{ Sample results, significancy of intra- and intergroup differences ${ }^{a}$} & \multicolumn{3}{|c|}{ ANOVA analysis $^{c}$} \\
\hline & Pre-PCI (1) & Post-PCI (2) & 1d-PCI (3) & $\begin{array}{c}\text { Separate } \\
\text { intragroup } \\
\mathrm{p}^{b}\end{array}$ & $\begin{array}{c}\text { Overall } \\
\text { intragroup } \\
\mathrm{p}\end{array}$ & $\begin{array}{c}\text { Between } \\
\text { groups } \\
\text { (intergroup p) }\end{array}$ & Interaction \\
\hline Cortisol (mmol/l) & & & & & 0.001 & 0.0499 & 0.0006 \\
\hline SAP & $375.81 \pm 173.66$ & $450.00 \pm 242.96$ & $360.43 \pm 183.71$ & 0.706 & & & \\
\hline ACS & $722.20 \pm 440.56$ & $595.80 \pm 440.84$ & $342.40 \pm 193.99$ & $0.006 *$ & & & \\
\hline ACS intragroup $p^{b}$ & 1 vs $2: 0.184$ & 2 vs 3: 0.060 & 1 vs 3:0.002* & & & & \\
\hline$S A P$ vs $A C S p^{b}$ & $0.018 *$ & 0.610 & 0.762 & & & & \\
\hline$A C T H(p g / m l)^{d}$ & & & & & 0.0007 & 0.940 & 0.023 \\
\hline SAP & $55.56 \pm 46.15$ & $89.80 \pm 76.65$ & $35.64 \pm 25.58$ & $0.014 *$ & & & \\
\hline SAP intragroup $p^{b}$ & 1 vs $2: 0.102$ & 2 vs 3: $0.004 *$ & 1 vs 3: $0.03 *$ & & & & \\
\hline ACS & $122.60 \pm 154.41$ & $140.00 \pm 158.03$ & $18.79 \pm 8.44$ & $0.0001 *$ & & & \\
\hline$A C S$ intragroup $p^{b}$ & 1 vs $2: 0.507$ & 2 vs 3:0.0001* & 1 vs $3: 0.0001^{*}$ & & & & \\
\hline$S A P$ vs $A C S p^{b}$ & 0.544 & 0.544 & $0.007 *$ & & & & \\
\hline
\end{tabular}

Plasma levels of cortisol and ACTH in patients with stable angina pectoris (SAP) or with acute coronary syndrome (ACS); directly before, directly after and on the following day of PCI (Pre, Post, 1d). In case of SAP, $n=21$ and for ACS, $n=20$.

${ }^{a}$ the intragroup and intergroup differences were calculated by special contrasts, based on estimated marginal means; after the correction by step-down Bonferroni method; significant intragroup differences ( $p<0.05$ values) are denoted by asterisks $\left({ }^{*}\right){ }^{b}$ in case of the presence of interaction (between groups), $p$ values are given after the correction by step-down Bonferroni method; ${ }^{c} p$ values of the two-way ANOVA; ${ }^{d}$ ACTH statistical analysis: log-transformed data (ANOVA was performed on the logarithm of data). 
Table A4. Plasma levels of special plasma markers and psychosocial scores of the SAP patients in study II.

\begin{tabular}{|l|l|c|}
\cline { 2 - 3 } \multicolumn{1}{c|}{} & \multicolumn{1}{c|}{ Parameter } & Value \\
\hline \multirow{3}{*}{ Inflammatory markers } & Cortisol (nmol/l) & $294.45 \pm 88.59$ \\
\cline { 2 - 3 } & Chromogranin A (ng/ml) & $178.81 \pm 105.50$ \\
\hline \multirow{3}{*}{ Psychosocial scores } & LL-37 (ng/ml) & $55.31 \pm 12.44$ \\
\cline { 2 - 3 } & IL-6 (pg/ml) & $1.83 \pm 1.35$ \\
\hline & Beck Depression Inventory & $7.4 \pm 9.4$ \\
\cline { 2 - 3 } & BSCI Global stress-coping & $-0.7 \pm 4.2$ \\
\cline { 2 - 3 } & BSCI Social support & $7.9 \pm 2.4$ \\
\cline { 2 - 3 } & BSCI Purpose and connection & $11.9 \pm 1.9$ \\
\hline
\end{tabular}

Variables are means \pm SD. BSCI: Rahe's Brief Stress and Coping Inventory.

( $n=23$, exceptions for sample number: $n=22$ for cortisol and LL-37.) 
Table A5. Plasma inflammatory markers with statistical analysis in SAP and ACS patients in study I. (mean \pm SD, $p$-values)

\begin{tabular}{|c|c|c|c|c|c|c|c|}
\hline \multirow[t]{2}{*}{ Parameters } & \multicolumn{4}{|c|}{ Sample results, significances of intra- and intergroup differences ${ }^{a}$} & \multicolumn{3}{|c|}{ ANOVA analysis $^{c}$} \\
\hline & Pre-PCI (1) & Post-PCI (2) & 1d-PCI (3) & $\begin{array}{c}\text { Separate } \\
\text { intragroup } \\
\mathrm{p}^{b}\end{array}$ & $\begin{array}{c}\text { Overall } \\
\text { intragroup } \mathrm{p}\end{array}$ & $\begin{array}{c}\text { Between } \\
\text { groups } \\
\text { (intergroup p) }\end{array}$ & Interaction \\
\hline Plasma lactoferrin (ng/ml) & & & & & $<0.0001$ & 0.267 & 0.826 \\
\hline SAP & $241.86 \pm 178.75$ & $306.32 \pm 193.49$ & $137.69 \pm 71.61$ & & & & \\
\hline ACS & $282.10 \pm 220.1$ & $365.72 \pm 220.47$ & $205.33 \pm 124.61$ & & & & \\
\hline SAP or ACS intragroup $p$ & 1 vs $2: 0.0008^{*}$ & 2 vs $3:<0.0001^{*}$ & 3 vs 1: $0.0008^{*}$ & & & & \\
\hline Plasma IL-6 (pg/ml) & & & & & $<0.0001$ & 0.057 & 0.482 \\
\hline SAP & $1.52 \pm 0.68$ & $2.11 \pm 1.16$ & $4.26 \pm 3.13$ & & & & \\
\hline ACS & $2.31 \pm 1.48$ & $2.80 \pm 1.88$ & $5.99 \pm 3.22$ & & & & \\
\hline SAP or ACS intragroup $p$ & 1 vs 2:0.0009* & 2 vs $3:<0.0001^{*}$ & 3 vs $1:<0.0001^{*}$ & & & & \\
\hline
\end{tabular}

Plasma levels of lactoferrin and IL-6 in patients with stable angina pectoris (SAP) or with acute coronary syndrome (ACS); directly before, directly after and on the following day of PCI (Pre, Post, 1d). In case of SAP, n=21 (IL-6: n=18), and for ACS, n= 20 (IL-6: n=19, except: Post: n=18).

${ }^{a}$ the intragroup and intergroup differences were calculated by special contrasts, based on estimated marginal means; after the correction by step-down Bonferroni method; significant intragroup differences ( $p<0.05$ values) are denoted by asterisks $\left({ }^{*}\right) ;{ }^{b}$ in case of the presence of interaction (between groups), $p$ values are given after the correction by step-down Bonferroni method; ${ }^{c} p$ values of the two-way ANOVA. 
Table A6. Appearance of special cell-surface inflammatory markers in SAP and ACS patients in study I. (mean \pm SD, $p$-values)

\begin{tabular}{|c|c|c|c|c|c|c|c|}
\hline \multirow[t]{2}{*}{ Parameters } & \multicolumn{4}{|c|}{ Sample results, significancy of intra- and intergroup differences ${ }^{a}$} & \multicolumn{3}{|c|}{ ANOVA analysis ${ }^{c}$} \\
\hline & Pre-PCI (1) & Post-PCI (2) & 1d-PCI (3) & $\begin{array}{c}\text { Separate } \\
\text { intragroup } \mathrm{p}^{b}\end{array}$ & $\begin{array}{c}\text { Overall } \\
\text { intragroup } \mathrm{p}\end{array}$ & $\begin{array}{l}\text { Between groups } \\
\text { (intergroup p) }\end{array}$ & Interaction \\
\hline L-selectin MFI & & & & & 0.092 & 0.735 & 0.732 \\
\hline SAP & $94.01 \pm 31.64$ & $93.64 \pm 27.01$ & $82.39 \pm 29.35$ & & & & \\
\hline ACS & $88.10 \pm 33.36$ & $89.64 \pm 34.89$ & $80.79 \pm 32.36$ & & & & \\
\hline L-selectin-bearing cells (\%) & & & & & 0.0002 & 0.613 & 0.147 \\
\hline SAP & $90.00 \pm 8.92$ & $93.71 \pm 4.65$ & $88.94 \pm 10.50$ & $0.001 *$ & & & \\
\hline SAP intragroup $p^{b}$ & 1 vs $2: 0.001^{*}$ & 2 vs 3:0.021* & $1 v s 3: 0.557$ & & & & \\
\hline ACS & $92.33 \pm 8.34$ & $93.53 \pm 8.00$ & $90.62 \pm 11.59$ & 0.302 & & & \\
\hline$S A P$ vs $A C S p^{b}$ & 1.000 & 1.000 & 1.000 & & & & \\
\hline CD15 MFI & & & & & 0.048 & 0.013 & 0.248 \\
\hline SAP & $75.78 \pm 42.52$ & $74.60 \pm 32.24$ & $68.19 \pm 30.47$ & 0.789 & & & \\
\hline ACS & $53.38 \pm 25.54$ & $58.61 \pm 27.25$ & $44.89 \pm 18.35$ & $0.032 *$ & & & \\
\hline ACS intragroup $p^{b}$ & 1 vs $2: 0.540$ & 2 vs 3: $0.032 *$ & 3 vs $1: 0.17$ & & & & \\
\hline$S A P$ vs ACS $p^{b}$ & 0.332 & 0.332 & $0.032 *$ & & & & \\
\hline CD15-bearing cells (\%) & & & & & $<0.0001$ & 0.199 & 0.759 \\
\hline SAP & $87.88 \pm 8.26$ & $89.25 \pm 8.29$ & $74.59 \pm 22.12$ & & & & \\
\hline ACS & $79.86 \pm 19.41$ & $84.16 \pm 18.16$ & $68.33 \pm 24.46$ & & & & \\
\hline SAP or ACS intragroup $p$ & 1 vs 2:0.018* & 2 vs 3: $<0.0001 *$ & 3 vs 1: $0.002 *$ & & & & \\
\hline Lactoferrin MFI & & & & & 0.001 & 0.017 & 0.436 \\
\hline SAP & $52.06 \pm 28.19$ & $52.38 \pm 25.25$ & $34.48 \pm 20.97$ & & & & \\
\hline ACS & $34.25 \pm 18.65$ & $38.54 \pm 16.51$ & $27.45 \pm 9.69$ & & & & \\
\hline SAP or ACS intragroup $p$ & 1 vs $2: 0.397$ & 2 vs 3:0.0006* & 3 vs $1: 0.01 *$ & & & & \\
\hline Lactoferrin-bearing cells (\%) & & & & & $<0.0001$ & 0.322 & 0.376 \\
\hline SAP & $35.80 \pm 25.14$ & $38.62 \pm 24.47$ & $23.26 \pm 21.23$ & & & & \\
\hline ACS & $28.57 \pm 20.31$ & $35.74 \pm 21.15$ & $15.07 \pm 13.98$ & & & & \\
\hline SAP or ACS intragroup $p$ & 1 vs 2:0.008* & 2 vs 3: $<0.0001 *$ & 3 vs 1: $<0.0001 *$ & & & & \\
\hline
\end{tabular}

Percentages of surface marker-bearing granulocytes and mean fluorescence intensities (MFI) of granulocytes in patients with stable angina pectoris (SAP) or with acute coronary syndrome (ACS); directly before, directly after and on the following day of PCI (Pre, Post, 1d). In case of SAP, n=21 and for ACS, n=20 (except: 1d granulocyte values, n=19). For ${ }^{a},{ }^{b}$ and ${ }^{c}$ : see Table A3. 


$$
\text { I. }
$$




\title{
ACTH- and Cortisol-Associated Neutrophil Modulation in Coronary Artery Disease Patients Undergoing Stent Implantation
}

\author{
Margit Keresztes ${ }^{1 *}$, Tamás Horváth ${ }^{2}$, Imre Ocsovszki ${ }^{1}$, Imre Földesi ${ }^{3}$, Gyöngyi Serfözö ${ }^{1}$, Krisztina Boda ${ }^{4}$ \\ Imre Ungi ${ }^{2}$
}

1 Department of Biochemistry, Medical Faculty, University of Szeged, Szeged, Hungary, 2 Invasive Cardiology Unit, Centre of Cardiology, Medical Faculty, University of Szeged, Szeged, Hungary, 3 1st Department of Internal Medicine, Medical Faculty, University of Szeged, Szeged, Hungary, 4 Department of Medical Physics and Informatics, Medical Faculty, University of Szeged, Szeged, Hungary

\begin{abstract}
Background: Psychosocial stress and activation of neutrophil granulocytes are increasingly recognized as major risk factors of coronary artery disease (CAD), but the possible relationship of these two factors in CAD patients is largely unexplored. Activation of neutrophils was reported to be associated with stenting; however, the issue of neutrophil state in connection with percutaneous coronary intervention (PCl) is incompletely understood from the aspect of stress and its hypothalamicpituitary-adrenal axis (HPA) background. Thus, we aimed to study cortisol- and ACTH-associated changes in granulocyte activation in patients undergoing $\mathrm{PCl}$.

Methodology/Principal Findings: Blood samples of 21 stable angina pectoris (SAP) and 20 acute coronary syndrome (ACS) patients were collected directly before (pre-PCI), after (post-PCI) and on the following day of PCI (1d-PCI). Granulocyte surface L-selectin, CD15 and (neutrophil-specific) lactoferrin were analysed by flow cytometry. Plasma cortisol, ACTH, and lactoferrin, IL- 6 were also assayed. In both groups, pre- and post-PCI ratios of lactoferrin-bearing neutrophils were relatively high, these percentages decreased substantially next day; similarly, $1 \mathrm{~d}-\mathrm{PCl}$ plasma lactoferrin was about half of the post-PCI value (all $p \leq 0.0001$ ). Post-PCI ACTH was reduced markedly next day, especially in ACS group (SAP: $p<0.01, A C S: p \leq 0.0001$ ). In ACS, elevated pre-PCl cortisol decreased considerably a day after stenting $(p<0.01)$; in pre-PCI samples, cortisol correlated with plasma lactoferrin $(r \sim 0.5, p<0.05)$. In $1 \mathrm{~d}-\mathrm{PCl}$ samples of both groups, ACTH showed negative associations with the ratio of lactoferrin-bearing neutrophils (SAP: $r=-0.601, p<0.005$; ACS: $r=-0.541, p<0.05$ ) and with plasma lactoferrin (SAP: $r=-0.435, p<0.05$; ACS: $r=-0.609, p<0.005$ ).

Conclusions/Significance: Pre- and post-PCl states were associated with increased percentage of activated/degranulated neutrophils indicated by elevated lactoferrin parameters, the $1 \mathrm{~d}-\mathrm{PCl}$ declines of which were associated with plasma ACTH in both groups. The correlation of plasma cortisol with plasma lactoferrin in the extremely stressed ACS before stenting, however, suggests an association of cortisol with neutrophil activation.
\end{abstract}

Citation: Keresztes M, Horváth T, Ocsovszki I, Földesi I, Serfózó G, et al. (2013) ACTH- and Cortisol-Associated Neutrophil Modulation in Coronary Artery Disease Patients Undergoing Stent Implantation. PLoS ONE 8(8): e71902. doi:10.1371/journal.pone.0071902

Editor: Cordula M. Stover, University of Leicester, United Kingdom

Received January 15, 2013; Accepted July 6, 2013; Published August 14, 2013

Copyright: 12013 Keresztes et al. This is an open-access article distributed under the terms of the Creative Commons Attribution License, which permits unrestricted use, distribution, and reproduction in any medium, provided the original author and source are credited.

Funding: The study was supported by in-house resources and by the PhD bench-fee of Gyöngyi Serfózó. The funders had no role in study design, data collection and analysis, decision to publish, or preparation of the manuscript.

Competing Interests: The authors have declared that no competing interests exist.

*E-mail: keresztes.margit@med.u-szeged.hu

\section{Introduction}

Although coronary artery disease $(\mathrm{CAD})$ and heart attack have been taught at medical schools for centuries, our comprehension of atherosclerosis as a disease of vascular inflammation is relatively new, and the underlying pathophysiological-pathobiochemical mechanisms are still not fully understood. In the initiation of this inflammatory reaction by oxidized LDL (a major, primary proatherogenic factor), mostly monocytes and macrophages are thought to have a primary role - in addition to endothelial cells, while dendritic cells and $T$ cells are supposed to act mainly in subsequent steps [ll 3 3. Recently, however, the unexpected importance of neutrophil granulocytes in the onset and progression of atherosclerosis has come to the limelight $\left[\begin{array}{ll}4 & 7\end{array}\right]$. Surpris- ingly, it was found that neutrophil count could be associated with the incidence of acute coronary events or may be an independent predictor of multiple stenosis in chronic stable angina [8 9 ]. Reports on the state of neutrophils in stable angina pectoris (SAP) are controversial $[4,10]$, while systemic neutrophil activation is a consistent finding in unstable angina $[11,12]$. Interestingly, it was shown that granulocytes are already primed in hyperlipidemic patients, thus, any second stimulus may trigger full activation of these leukocytes [13].

There is an increasing evidence, that psychosocial stress could play a major part in the development of coronary artery disease. The great INTERHEART study revealed that psychosocial stress is to be regarded as a main risk factor for myocardial infarction 
$[14,15]$. Furthermore, patients with acute coronary syndrome (ACS) are usually extremely stressed, and stable CAD patients may also feel tension e.g. before-during PGI [16]. Thus, it could be most important, that psychological stress itself could result in activation of neutrophils in anxious patients and even in healthy individuals $\left[\begin{array}{ll}17 & 19\end{array}\right]$.

In neuroendocrine-immune regulation of the stress response/ state, HPA axis has an outstanding role via GRH, ACTH and cortisol [20]. In addition to the well-known role of cytokine/ chemokine receptors, neutrophil behaviour and homeostasis are regulated also by stress hormones, e.g. by glucocorticoids via specific receptors $[5,7,21]$. Although cortisol and its analogs are known and widely used as anti-inflammatory agents and immune suppressors, (morning) plasma cortisol levels were reported to correlate with the severity of coronary atherosclerosis in US Air Force aircrew members and in patients with suspected CAD [22 23]. Furthermore, cortisol stress reactivity was found to be associated with coronary artery calcification in healthy men and women [24].

Cytokines are suggested to have a central importance both in stress/depression, in atherosclerosis and in CAD; particularly, the role of IL- 6 is emphasized as a main regulator of the systemic inflammatory response and as a most potent stimulator of the HPA axis $[20,25,26]$. Stenting/PGI was reported to trigger rapid, transient neutrophil activation, followed by the release of IL- 6 and IL-8 in unstable angina; a similar post-PGI elevation of cytokines was reported also in stable angina $[27,28]$.

So far, the issue of neutrophil activation state in connection with PCI is largely unexplored from the aspect of stress and its HPA background. Therefore, we set out to investigate the state of granulocytes in patients with SAP or ACS - before, directly after and on the next day of PCI; and we wished to examine the possible associations between granulocyte state and plasma ACTH or cortisol. Our results suggest an association between plasma cortisol and a neutrophil degranulation marker (in plasma) in the grossly stressed ACS patients before stenting, while the decrease in the percentage of activated neutrophils was associated with plasma ACTH in both patient groups on the next day of PGI.

\section{Materials and Methods}

\section{Patients}

25 SAP and 20 AGS patients were enrolled for our prospective study. Patients (over 18 years) were eligible if they had characteristic symptoms and findings of either stable angina (with positive stress test) or acute coronary syndrome. (In the ACS group, 6 patients had unstable angina, and 14 patients were diagnosed to have acute myocardial infarction/with increased cardiac troponin $\mathrm{T}$ level/; from the latter group, 6 subjects had EGG with ST elevation, while no ST elevations were seen in the EGG of 5 patients.).

For inclusion, at least one significant stenosis (diameter $>50 \%$ ) was to be demonstrated by coronary angiography, as suggested by Videm et al. [10]; angiography was followed by stenting in a single vessel. (In both groups, the patients were included if the PCI could be performed between 8 a.m. 2 p.m., as the flow cytometry procedure was to be finished in the same afternoon.).

Exclusion criteria included prior bypass surgery, cardiogenic shock, malignancy, any immunological disorders or acute inflammations, immunosuppressive or anti-inflammatory treatment (except low-dose aspirin), recent major trauma/surgery, drug/ alcohol abuse, poor mental function. Four stable patients' samples could not be processed (blood clotting disturbances and sample management problems); thus, our study involved 41 patients (27/
14 males/females; age 3387 years). All patients took aspirin in low doses during the study; statins, antihypertensives, sedatives were not discontinued. Clopidogrel $(600 \mathrm{mg}$ loading dose, 75 $150 \mathrm{mg}$ maintenance dose) was given before and after PCI; all patients were administered heparin $(70100 \mathrm{IU} / \mathrm{kg}$ ) during PGI.

The study protocol was approved in advance by the Regional Medical Ethics Committee (Human Investigation Review Board, University of Szeged, Albert Szent-Györgyi Clinical Centre; Ref No: 37/2005). Most participants gave full written consent to participate before enrolling, and after giving detailed informations on the study from the aspect of participants (interactive talk, written study description). In serious acute cases, the patients gave informed written consent after the emergency life-saving intervention was finished. The investigation conformed to the principles of the Declaration of Helsinki.

\section{Blood Sampling and Laboratory Methods}

Blood samples were collected immediately before PGI (prePCI), immediately after PCI (post-PCI), and on the following day (ld-PCI). (The mean time of PGI was about $45 \mathrm{~min}$, with a range between $30 \mathrm{~min}$ and one hour.) The ld-PCI samples were collected routinely between $89 \mathrm{~h}$ a.m. in each case. Blood collection for routine blood analysis (total leukocyte count, neutrophil fraction and lipid panel) was performed at admission for acute patients, and before admission for the stable angina pectoris group (to check exclusion criteria like acute inflammation). CK values were assayed in ld-PCI samples, in order to monitor (diagnose) heart attack in the AGS group, and to check the occurrance of acute procedural myocardial infarction (a possible consequence of $\mathrm{PCI}$ in the SAP patients.

Blood cell counts, total cholesterol, triglycerides, HDL-cholesterol and CK activity were measured by standard protocols in automatic cell counter/analyzers (Roche); LDL-cholesterol was calculated (Friedewald formula). Reference ranges: total leukocyte count: $3.911 .110^{9} / 1$, neutrophil fraction: $44.068 .0 \%$, total cholesterol: $<5.2 \mathrm{mmol} / 1$, triglycerides: $<2.0 \mathrm{mmol} / 1$, HDLcholesterol: $>1.0 \mathrm{mmol} / 1$, LDL-cholesterol: $<3.0 \mathrm{mmol} / \mathrm{l}$, GK activity: $<200 \mathrm{U} / 1$. Cardiac troponin $\Upsilon$ assay was performed by a qualitative, rapid dry chemistry test (Roche) using heparinized whole blood, and it was evaluated visually.

ACTH, cortisol, lactoferrin and IL- 6 were assayed in EDTAplasma, plasma aliquots were stored at $-80^{\circ} \mathrm{C}$ for these analyses. ACTH concentrations were determined by a chemiluminescent immunoassay (LKAGl, Immulite 1000; Siemens Healthcare Diagnostics, UK); reference range: $060 \mathrm{pg} / \mathrm{ml}$. Cortisol was assayed by radioimmunoassay (DSL-2100; Diagnostic Systems Laboratories, Webster, TX); reference range: $160620 \mathrm{nmol} / 1$ Plasma lactoferrin was determined by an "in-house" ELISA kit, developed according to Antonsen [29]. Briefly, microplates were coated overnight with rabbit anti-human lactoferrin $(3.7 \mathrm{mg} / \mathrm{l}$; Dako, Denmark) at $4^{\circ} \mathrm{G}$. After washing twice, plasma samples were applied in duplicates (in wells: ten-fold dilution), and incubated for $1 \mathrm{~h}$ at room temperature. Incubation with antilactoferrin-peroxidase was carried out similarly, after five washing steps. The conjugate was prepared from Dako antibody and from horseradish peroxidase (Calbiochem, USA) according to Wilson and Nakane [30]; it was used in 4000-fold diluted form. Following washing steps, plates were developed with TMB (BD Biosciences, USA), and reaction was stopped by $4 \mathrm{~N}$ sulfuric acid. Human lactoferrin (Sigma, USA) served as standard; reference range/ median for plasma lactoferrin: 40 200/90 ng/ml [29]. IL-6 was assayed by a commercial ELISA kit (BMS213/2MST; Bender MedSystems, Austria); normal/reference maximum value: $14.1 \mathrm{pg} / \mathrm{ml}$ (mean: $1.3 \pm 3.2 \mathrm{pg} / \mathrm{ml}$ ). 
The presence of surface granulocyte activation markers: Lselectin, CD15 and lactoferrin was analysed by flow cytometry using an indirect immunofluorescent method; the process for labelling and hemolysis was started within 2 hours after blood collection. The protocol employed was the same as described before [18], except that a different CD15 monoclonal antibody was applied (Dako, $8.5 \mathrm{mg} / \mathrm{l}$ ). (Other primary antibodies: a polyclonal anti-lactoferrin (200 mg/1, ICN), and a monoclonal anti-L-selectin (47 mg/l) (Dako); secondary antibodies: anti-rabbit $(10 \mathrm{mg} / \mathrm{l})$ or anti-mouse $(20 \mathrm{mg} / \mathrm{l})$ FITG-labelled antibodies (Dako); negative controls: normal rabbit serum or isotype-specific normal mouse Ig (Dako)). Immunolabelling of whole-blood EDTA samples was initiated within $1 \mathrm{~h}$. Hemolysis was carried out after immunostaining (Lysing kit; Biodesign, USA). Investigations were carried out with a FACStar Plus Becton-Dickinson equipment; only the population of granulocytes was analysed from leukocytes. Granulocytes were gated on the basis of their characteristic forward- (FS) and side-scatter (SS) features; as they have relatively high side scatter, granulocytes could be easily distinguished from the two other, main leukocyte populations: i.e. from lymphocytes, and monocytes. (The elevated side scatter is related to the granularity of these leukocytes; the forward scatter is roughly proportional to the diameter of the cell.) As granulocytes constitute the predominant leukocyte fraction, we could easily delimit the area of their population with high FS in the flow cytometry procedure. Granulocytes with cell surface lactoferrin were identified as activated neutrophils, since lactoferrin is specific for neutrophils, and it can gain access to the cell surface only after degranulation triggered by cell activation [31 33]. 10000 events/ tube were recorded in granulocyte populations. All measurements were preceded by standard equipment calibrations, and each detection series started with setting the background intensity level using the proper negative controls. Ratios of labelled granulocytes (\% of cells/granulocytes carrying/bearing labelled markers on cell surface) and mean fluorescence intensities (MFI, related to mean quantity of labelled molecules/cell) are shown.

\section{Statistical Analysis}

For continuous variables, data are presented as mean \pm standard deviation (SD) in Table 1 (upper part), and in the supplementary table (Table S1); in case of Figures, mean \pm standard error of means (SEM) is used. General characteristics of the subject groups were compared by two-sample Student's t-test. In case of categorical variables, number of subjects ( $\mathrm{n}$ ) and percentage (\%) were given, and the groups were compared by Fisher's exact test (Table 1, bottom). Distribution was checked by Kolmogorov-Smirnov statistics; in case of skewed distribution (ACTH), further analysis was performed on log-transformed data.

In our analysis, there were two main factors: the time (pre-PCI, post-PCI and ld-PCI) as within-group ("intragroup") factor for repeated measurements and the two groups as between-subject ("intergroup") factor for independent samples (Table S1). For over time comparisons (pre-PGI, post-PGI, and ld-PGI samples), twoway repeated measurements ANOVA was used. The resulting $p$ values were for overall intragroup $p$, between groups/intergroup $p$, and one for their interaction. Significant value of interaction allowed also between-group (SAP vs ACS) comparisons at each timepoint. When the $p$ value of interaction was relatively low: $0.3>p>0.05$, the presence of interaction was assumed to allow intergroup comparisons in these special statistical cases. Significancy of the separate intragroup $p$ allowed further pairwise comparisons of the repeated measurements within each group. All the pairwise comparisons are modified t-tests, these and the separate intragroup $p$-s were calculated by the software based on
Table 1. Characteristics of the patient groups and routine blood analysis.

\begin{tabular}{llll}
\hline & & & \\
\hline Variable & SAP (n=21) & ACS (n=20) & $P$ \\
\hline Age (y) & $64.1+10.3$ & $63.2+13.6$ & 0.81 \\
Total leukocyte count (G/) & $7.02+1.41$ & $8.37+3.02$ & 0.08 \\
Neutrophil fraction (\%) & $62.62+7.46$ & $65.83+8.18$ & 0.20 \\
Cholesterol (mmol/) & $4.34+1.28$ & $5.01+1.10$ & 0.11 \\
HDL-cholesterol (mmol/l) & $1.29+0.45$ & $1.30+0.41$ & 0.95 \\
\hline LDL-cholesterol (mmol/l) & $2.36+1.04$ & $3.13+1.04$ & $0.043^{*}$ \\
Triglycerides (mmol/) & $1.60+0.62$ & $1.35+1.01$ & 0.36 \\
CK (U/l) & $175.0+286.6$ & $669.8+715.7$ & $0.008^{*}$ \\
Male gender (n, \%) & $13(61.9)$ & $14(70.0)$ & 0.74 \\
Diabetes mellitus type II (n, \%) & $5(23.8)$ & $6(30.0)$ & 0.73 \\
Hypertension (n, \%) & $16(76.2)$ & $16(80.0)$ & 1.00 \\
Statins use (n, \%) & $16(76.2)$ & $15(75.0)$ & 1.00 \\
Antihypertensives use (n, \%) & $21(100)$ & $17(85.0)$ & 0.11 \\
\hline Sedatives use (n, \%) & $7(33.3)$ & $7(35.0)$ & 1.00 \\
\hline
\end{tabular}

SAP: stable angina pectoris, ACS: acute coronary syndrome patients.

Values of continuous variables are means $+S D$, values of categorical variables are $n(\%)$.

Exceptions for sample numbers in lipid panel: total cholesterol, triglycerides (SAP: $n=19$, ACS: $n=16$ ); HDL-cholesterol (SAP: $n=18$, ACS: $n=16$ ); LDLcholesterol (SAP: $n=18$, ACS: $n=15$ ). $p<0.05$ values (significant differences between the two groups) are denoted by asterisks $\left(^{*}\right)$.

doi:10.1371/journal.pone.0071902.t001

estimated marginal means. The step-down Bonferroni adjustment was employed for multiple comparisons to keep the familywise type I error rate on $\alpha=0.05$ level. It is well known, that responses measured on the same subject are usually correlated; also, variances of repeated measures often change with time. We used a mixed model, where this correlation can be modelled. For our data, a general unstructured form was chosen for within-subject variance covariance matrix. Heterogeneity of group-variances was also taken into account. To examine the relationship between the stress hormones and selected inflammatory markers, Spearman's coefficient of correlation and its significance were calculated (Table 2). Statistical analyses were carried out with SPSS (version 15, SPSS Inc., Chicago) and SAS (v9.1, SAS Institute, Cary, NG) softwares. All tests were two-sided, and $p<0.05$ was considered to be statistically significant.

\section{Results}

\section{General Characteristics of the Patient Groups and Routine Blood Data}

Our study involved 21 stable angina pectoris and 20 acute coronary syndrome patients with a majority of males in both groups (Table 1). The only between-group differences found were: slightly increased LDL-cholesterol and markedly elevated CK activity in the ACS group. All other presented laboratory parameters were within the normal ranges.

\section{Stress Hormones (Cortisol, ACTH), Surface Activation Markers of Granulocytes (L-selectin, CD15, Lactoferrin) and Inflammatory Markers in Plasma (Lactoferrin, IL-6)}

On admittance to the clinic, ACS patients were in a stressed state, with raised plasma cortisol and grossly elevated ACTH concentration; these values decreased dramatically next day after 
Table 2. Correlations between stress hormones and selected inflammatory markers.

\begin{tabular}{|c|c|c|c|}
\hline $\begin{array}{l}\text { Correlations between simultaneous } \\
\text { data (patient group, time, parameter) }\end{array}$ & $\begin{array}{l}\text { Surface lactoferrin-bearing } \\
\text { granulocytes }(\mathbf{r})\end{array}$ & Plasma lactoferrin (r) & II-6 (r) \\
\hline SAP - pre-PCI - Cortisol & $0.051(p=0.827)$ & $0.103(p=0.658)$ & $-0.218(p=0.385)$ \\
\hline SAP - pre-PCI - ACTH & $-0.283(p=0.213)$ & $-0.158(p=0.493)$ & $-0.483^{*}(p=0.042)$ \\
\hline ACS - pre-PCI - Cortisol & $0.391(p=0.088)$ & $0.499^{*}(p=0.025)$ & $-0.135(p=0.581)$ \\
\hline ACS - pre-PCI - ACTH & $0.364(p=0.115)$ & $0.398(p=0.082)$ & $-0.298(p=0.215)$ \\
\hline SAP - 1d-PCI - Cortisol & $-0.192(p=0.404)$ & $-0.015(p=0.950)$ & $0.269(p=0.280)$ \\
\hline SAP - 1d-PCI - ACTH & $-0.601^{*}(p=0.004)$ & $-0.435^{*}(p=0.049)$ & $0.024(p=0.926)$ \\
\hline ACS - 1d-PCI - Cortisol & $0.281(p=0.244)$ & $0.250(p=0.289)$ & $0.254(p=0.293)$ \\
\hline ACS - 1d-PCI - ACTH & $-0.541 *(p=0.017)$ & $-0.609^{*}(p=0.004)$ & $-0.068(p=0.783)$ \\
\hline
\end{tabular}

SAP: stable angina pectoris, ACS: acute coronary syndrome patients; pre- $\mathrm{PCl}$ : directly before, and 1d-PCl: on the following day of $\mathrm{PCl}$ (stenting).

$r$ : Spearman's rank correlation coefficient; asterisks $\left(^{*}\right)$ denote significancy of correlations $(p<0.05)$.

SAP: $n=21$, ACS $n=20$, except for IL-6 (ACS pre- and 1-d-PCI: $n=19$ ) and for percentage of surface lactoferrin-bearing cells (in ACS patients 1-day after PCI: $n=19$ ). doi:10.1371/journal.pone.0071902.t002

PCI: to about $50 \%$ (cortisol: $p<0.01$ ), and to $15 \%$ (ACTH: $p=0.0001)$ (Fig. 1, Table S1). Cortisol values of SAP patients were in the normal range, and did not change before/after PCI. However, a substantial decline of the ACTH level was observed also in these patients the day after PCI (pre-PCI/1d-PCI: $p<0.05$, post-PCI/1d-PCI: $p<0.01$ ); interestingly, the $1 \mathrm{~d}-\mathrm{ACTH}$ value was significantly higher in the SAP group than in the ACS one $(p<0.05)$. Significant (statistical) interactions could be demonstrated only in the case of the stress hormones (cortisol: $p<0.001$, ACTH: $p<0.05$ ) that allowed between-group comparisons.

Considering cell surface appearances of L-selectin and CD15 (a selectin ligand), no (L-selectin MFI) or only mild changes could be seen in a time- and/or intervention-dependent manner (Fig. 2, Table S1). In the SAP group, proportion of L-selectin-carrier granulocytes slightly increased during PCI $(p<0.01)$, and decreased next day, declining to about its initial value $(p<0.05)$. Similarly to the previous parameter, no distinction could be made between groups considering the slightly rising (pre-PCI/post-PCI: $p<0.05$ ) and then mildly declining (post-PCI/1d-PCI: $p<0.0001$ ) ratios of CD15-bearing granulocytes. It is to be noted, that the 1d-
PCI percentage of the CD15-bearing granulocytes was lower than the initial one $(p<0.01)$ in both groups. Concerning CD15 MFI, it was reduced somewhat after PCI $(p<0.05)$ in the ACS group, and difference between groups could be shown in 1d-PCI CD15 MFI $(p<0.05)$.

More marked changes were seen in case of neutrophil-specific surface lactoferrin (Fig. 2, Table S1). The ratio of surface lactoferrin-bearing granulocytes approximately halved following PCI (post-PCI/1d-PCI: $p<0.0001$ ). The pre-PCI values were high relatively to the 1d-PCI ones, and elevated slightly during the intervention $(p<0.01)$; interestingly, these 1d-PCI percentages were considerably lower than the pre-PCI ones $(p<0.0001)$. Less substantial alterations were found in case of lactoferrin MFI after PCI (post-PCI/1d-PCI: $p<0.001$ ). Similarly to the proportion of surface lactoferrin-bearing granulocytes, plasma lactoferrin approximately halved till the next day after PCI $(p<0.0001)$; these 1d-PCI values were significantly lower than the initial ones $(p<0.001)$ (Fig. 3). In post-PCI samples, a moderate increase was observed $(p<0.001)$. Pre- and post-PCI plasma lactoferrin concentrations were over the upper limit of the physiological
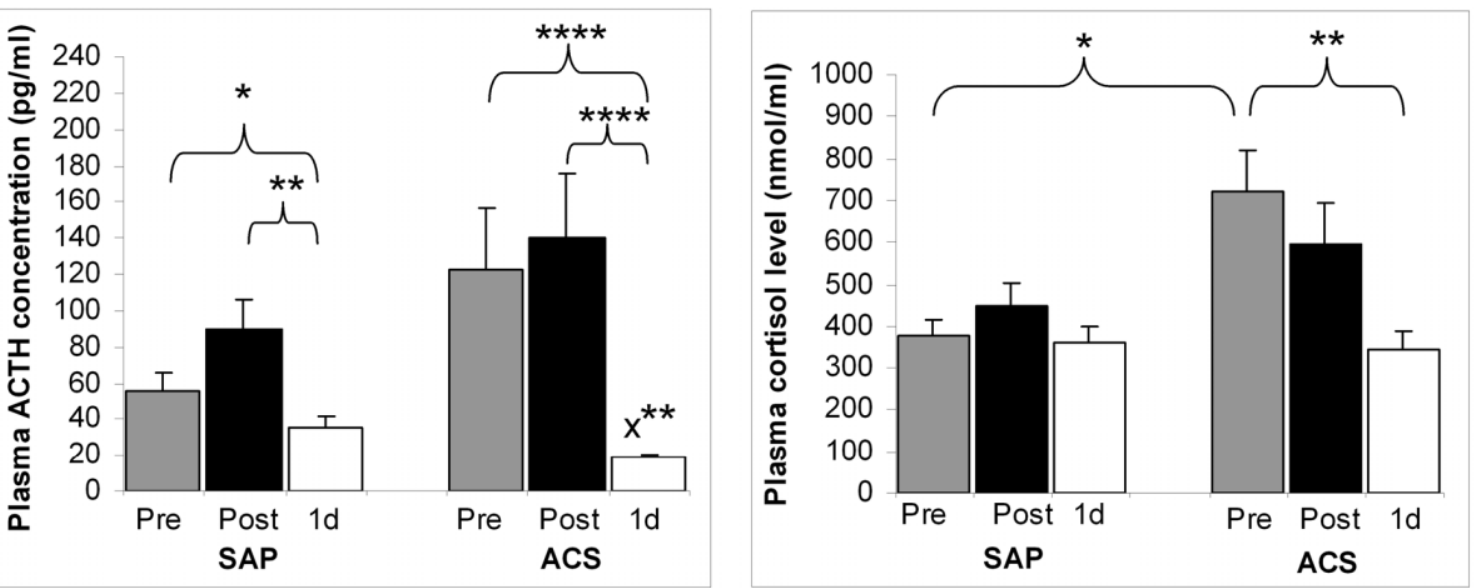

Figure 1. Plasma levels of ACTH and cortisol in CAD patients undergoing PCI. Blood samples were collected from stable angina pectoris (SAP) and from acute coronary syndrome (ACS) patients directly before (Pre), directly after (Post) and on the following day (1d) of PCI. Data are presented as mean $\pm \mathrm{SEM}$; in case of SAP, $\mathrm{n}=21$, and for $\mathrm{ACS}, \mathrm{n}=20$. Significant intragroup differences: Pre/1d, Post/1d; $p^{*}<0.05, p^{* *}<0.01, p^{* * * *}$ $\leq 0.0001$; significant intergroup differences (SAP vs ACS) for ACTH: $1 \mathrm{~d}, p \mathrm{x}^{* *}=p^{* *}<0.01$; for cortisol: Pre, $p^{*}<0.05$. doi:10.1371/journal.pone.0071902.g001 

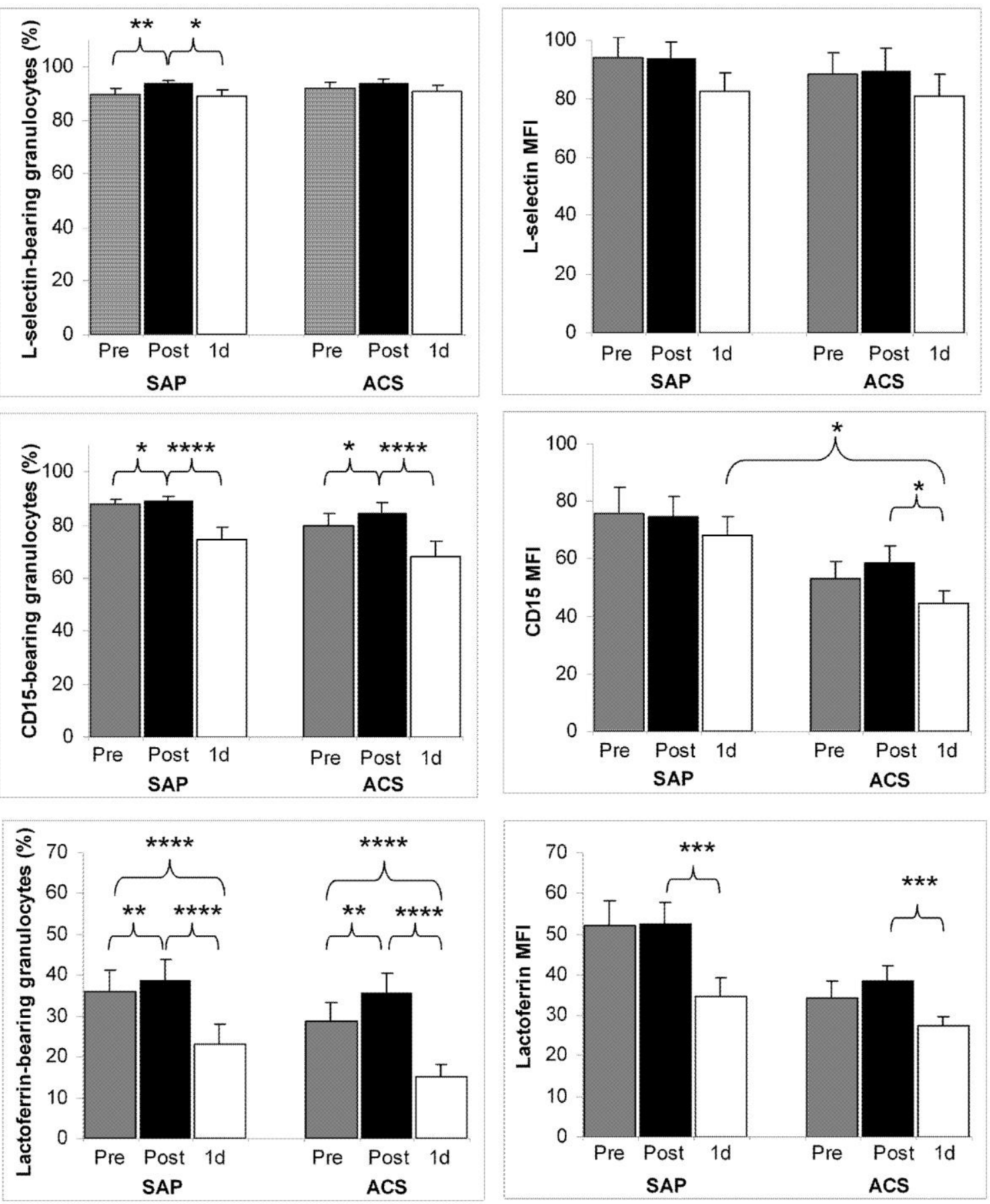

Figure 2. Flow cytometry analysis of granulocytes in CAD patients undergoing PCI. Percentages of marker-bearing granulocytes and mean fluorescence intensities (MFI) of granulocytes reflect the cell surface appearance of activation markers directly before, directly after and on the following day of $\mathrm{PCl}$ (Pre, Post, 1d) in patients with stable angina pectoris (SAP) or with acute coronary syndrome (ACS). Data are mean \pm SEM; in case of SAP, $\mathrm{n}=21$, and for ACS, $\mathrm{n}=20$ (ACS exception: $1 \mathrm{~d}$ values, $\mathrm{n}=19$ ). Significant differences: $p^{*}<0.05, p^{* *}<0.01, p^{* * *}<0.001, p^{* * * *} \leq 0.0001$. doi:10.1371/journal.pone.0071902.g002

range $(200 \mathrm{ng} / \mathrm{ml}$ ). Plasma IL-6 displayed a different pattern of changes: a mild increase immediately after intervention was followed by an about 2-fold elevation (pre-PCI/post-PCI: $p<0.001$, post-PCI/1d-PCI: $p<0.0001$ ).
Correlations between Stress Hormones (Cortisol, ACTH) and Selected Markers: Percentage of Surface Lactoferrinbearing Neutrophils, Plasma Lactoferrin, IL-6

As the most substantial alterations were found in the ratio of surface lactoferrin-bearing cells, plasma lactoferrin and IL-6, 

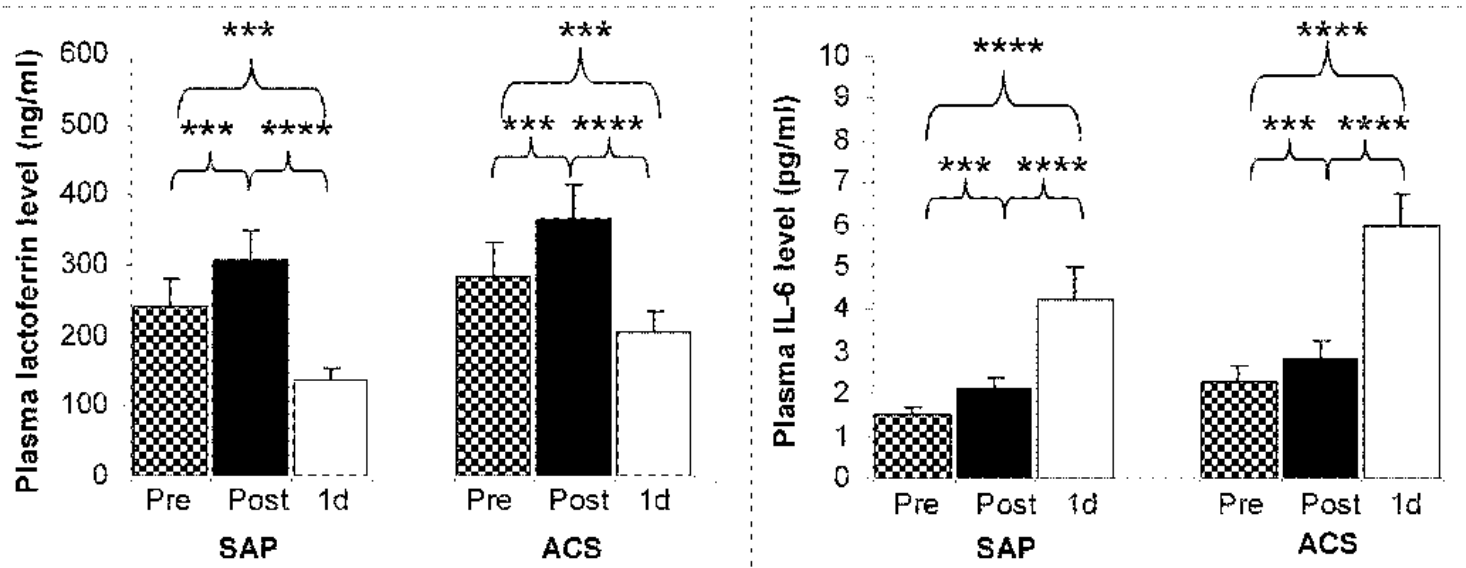

Figure 3. Plasma levels of lactoferrin and IL-6 in CAD patients undergoing PCI. Blood samples were collected from stable angina pectoris (SAP) and from acute coronary syndrome (ACS) patients directly before (Pre), directly after (Post) and on the following day (1d) of PCl. Data are presented as mean + SEM; in case of SAP, $n=21$ (IL-6: $n=18)$, and for ACS, $n=20$ (IL-6: $n=19$, except: Post: $n=18)$. Significant differences: $p^{* * *}$ $<0.001, p^{* * * *} \leq 0.0001$

doi:10.1371/journal.pone.0071902.g003

correlations among these inflammatory markers and the assayed sires hormones were tested Table 2\%. In ld-PCI samples of bull groups, plasma AC:I'H showed inverse associations with the ratio of lacoferrin-bearing neurophils $(\mathrm{S} A \mathrm{P}: r=-0.601, f<0.005$; ACS: $r=-0.541, p<0.05)$ and with plasma lactoforrin $(S \mathrm{AP}$ : $r=-0.435, f<0.05 ; \Delta C S: r=-0.609, p<0.005 ;$. Tn addition, ACTH correlated negatively will IL-6 in post-PCI samples of both groups (SAP: $r=-0.187, p<0.05 ;$ ACS: $r=-0.505, p<0.05$, and in the pre-PCT sample of ule SAP group $(r=-0.489, p<0.05)$. In contrast, cortisol was positively associated with plasma lactoferria in $\mathrm{ACS}$ pantents at the start $r=0.499, \ldots<0.05)$.

\section{Discussion}

Our results suggest that pre- and post-PCI states could be associated with increased perentage of acivated/degranuated neutrophils releasing lactoferrin. In the highly stressed $\mathrm{ACs}$ paticnss wibh clevated HPA axis activity, plasma lactuferrin correlated with plasma cortisul before PCII. The following day after PCI, the ratio of surface lactoferrin-carrier granulocytes and plasta lactofergin decreased in bot groups that conld reflect the negative immunc-modulatory effect of $\mathrm{AC}^{\prime} \mathrm{l}^{\prime} \mathrm{H}$ as suggested by negative correlationsi.

Our ACS patients appeared to be in an extremely stressed state befure PCI indicated by highly clevated plasma cortisol, whicl was substantially reduced on the day after PCl. The non-reactive, unchanged corlisol level of SAP pautesis could be due ur a previously overloaded, exhausted HPA axis [31]. In ACS, the overactivated HPA axis suitcled io a much decreased, normal functioning state during the hours after stenting: the final low plasma ACTH may reflect a gross relief following PCI. In SAP group, dic somewhat decreased ld-PCI ACITH valuc could mark a more relaxed state relatively to the initial one. $A s$ the peals of ACTH and cortisol conecontrations is blood are in llo carly morning period/before $6 \mathrm{a} \mathrm{m} . /$, the statistically) significant decreases in plasma ACTH and cortisol in the 1d-PC:T sanples (collected routinely between $8-9$ a.m.) are most probably not related to the diurnal fluctuation.

I-selectin is constitutively expressed in the leukocyte plasma mombranc. Activation is thuoght to lead mosty to its deavage/ shedding from the cell surface, like in unstable angina pectoris
[28]: however, increased cell surface appearance could also accompany acivation, eg. after medanical trauma [35]. In lue ratio of L-sclectin-bearing granulocytes of SAP paticnts, the slight increase during PCl followed by a moderate decrease is not remarkable (athengh statistically significant; this might indicate honcostatic alterations in these activated leulocytes silcedding balanecd by reappearance?

CID15 (Lewis-X) is a Letratsacharide ligand (to sclectins) prescht on all myeloid cells; in neutrophils, it can be mobilized from azuroploilic/primary granules [36]. The statistically siguificant. slighte increase in post-PCI ratio of CD15-bearing cells is not considerable; it could retlect slight granulocyte activation during the intervention in both patient groups. The moderate downregulation on the following day, howeyer, indicates a less activated state, similarly to the granulocytes of anxious pationts in a relatively relaxed condition in our previous study [18]

Lactoferrin represents at specific activation marker of neutrophils. which can be bound wo ect surface and ean gain acess 1.0 the cifculat ion only after ins release from secondary granules upon cel] activation [31-33]. It is worth noting, that ${ }^{105}$ I-labelled lactoterrin was shown to be rapidly cleared from the circulation, with al lactoferrin beisug removed willion 7 lasurs after injection in humans [37]. The pronounced reductions in cell surface and plasma lactoferrin paraneters mark a less activated state of neutrophils a day after PCI, independently of patient. group. Directly after intervention, a mild, not remarkable increase was ubserved in the propurtion of matker-carrice ncutrophils in boll paticil grueps, similarly to the case of (CI) 5 . These intervention-related moderate elauges could be observed also in plasma lactuferrin, which rellect linited neutruphil attivation, Gacts ot al. described a steep rise of plasma lactoferrin in coronary blood immediately afier PCI in patients will unstable angina. that was reversed in 6 hous [287. In our study, the pre-PCI values of sufface lactufestinbcaring granulocytes were also high $>25 \%$, indicating cnhanecd percentage of activaned neutrophits in CAD. Our results on elevated pre-PCI plasma latenferin in both groups correspond to those of Viden ct al.. who proposed raiscd plasma lactoferrin as an excellent marker for significant atherosclerotic coronary stenosis and found it to be more reliable, than plasma myeloperoxidase andethey neutrophil activation marker) [10]. Even morc, increased bascline plasma lactoforin was suggested to predict 
long-term risk for fatal $\mathrm{CAD}$ in patients with newly diagnosed diabetes [38]. As plasma lactoferrin and the percentage of surface lactoferrin-carrier granulocytes showed similar, marked changes in our patient groups, both may be used as "neutrophil activity sensors" in CAD patients. From the practical point of view, however, plasma lactoferrin assays appear to be more favourable; (less expensive tests which probably could be automated).

Onset of acute phase reaction was seen as a rising plasma IL-6, particularly enhanced a day after PCI, when activation level of neutrophils was already reduced. Similarly, a decrease of plasma lactoferrin and myeloperoxidase and increase of IL-6 were observed during 612 hours after stenting in unstable angina [28]. Caixeta et al. found a statistically significant, but moderate increase of IL- 6 level at $6 \mathrm{~h}$ after PCI both in unstable and in stable angina patients followed by a decline; however, this study may have lost the peak at $24 \mathrm{~h}$ as suggested [27]. It is worth noting, that psychological stress was found to have strong relationship with circulating level of IL-6 in treated HIV-infected individuals [39].

Our principal finding is that correlations could exist among the neutrophil activation state and plasma cortisol and/or ACTH in GAD patients. It is plausible to suppose that AGTH could be a suppressor of neutrophil activation/degranulation: its plasma level was inversely associated with the ratio of surface lactoferrinbearing granulocytes and plasma lactoferrin in both groups a day after PGI. This is in agreement with the concept of tonal inhibition of immune/inflammatory responsiveness by ACTH [40]. Correlation of cortisol with plasma lactoferrin before PGI in AGS seems to be controversial, as cortisol and its analogs are known as antiinflammatory agents and immune suppressors. However, glucocorticoids were also reported to activate human neutrophils in vitro; and the relatively high ratio of $\alpha$ to $\beta$ type of glucocorticoid receptors is suggested to be responsible for neutrophil insensitivity to apoptosis induction by these hormones $[21,41]$. Furthermore, as morning plasma cortisol levels were found to correlate with the severity of coronary atherosclerosis in US Air Force aircrew members and in patients with suspected $\mathrm{CAD}$, cortisol may be considered as a proatherogenic stress hormone [22 23]. This notion is supported by some investigations finding that selective inhibitors of the enzyme specifically involved in cortisol synthesis (11ß-hydroxysteroid dehydrogenase) could be useful in prevention of atherosclerosis [42 43].

Neutrophil granulocytes could be activated as part of an ancient adaptation/survival process in case of danger/stress [44]. Stimulation of neutrophils could be involved in the initiation of atherosclerosis in a severalfold way. Hypochlorous acid (HOCl) produced by neutrophil-specific myeloperoxidase was reported to play a critical role in the production of oxidized LDL, that further stimulates neutrophils leading to activation of respiratory burst (production of reactive oxygen species) and to degranulation (release of enzymes, some adhesion receptors, lactoferrin etc.). Interestingly, HDL apolipoprotein AI and vitamin C could be protective [45 46]. Additionally, alfa-defensins (neutrophil peptides) could be also involved in the cardiovascular inflammatory reaction [47].

There are some limitations to be discussed. First, the exact mechanisms in the background of plasma ACTH/cortisol

\section{References}

1. Plutzky $J$ (2001) Inflammatory pathways in atherosclerosis and acute coronary syndromes. Am J Cardiol 88(suppl): $10 \mathrm{~K} 15 \mathrm{~K}$.

2. Andersson J, Libby P, Hansson GK (2010) Adaptive immunity and atherosclerosis. Cilin Immunol 134: 3346 . correlations with the neutrophil activation state (inflammatory state) could not be clarified in this clinical study. Second, several characteristics of the patient groups might confound the described changes: e.g. life style factors, age, sex, and pre-existent illnesses. It is to be noted that metabolic syndrome could affect ACTH levels; in addition, this syndrome is widely known to be often accompanied by CAD [ $\left.\begin{array}{ll}48 & 49\end{array}\right]$. However, our patient groups functioned as auto-controlled statistical units (i.e. repeated measurements within-group, over-time comparisons), therefore, the confounding factors listed above had probably limited influence. The relatively small sample size must be also considered; still, the high levels of statistical significances support the validity of our results.

Despite the apparent limitations, our study offers interesting insights into the dynamically changing neutrophil behaviour in CAD patients undergoing stenting. We showed that neutrophil activation could be enhanced already before stenting, and that neutrophils could be less activated next day after PCI - after a mild, transitory increase in their activation state directly after stenting. Plasma lactoferrin appeared to be a sensitive and valuable marker of neutrophil activation, which deserves detailed methodological studies to evaluate it as a risk marker for CAD. To our best knowledge, we are the first to find correlations between plasma ACTH, cortisol and neutrophil activation/degranulation state in CAD patients before and/or after stenting; further studies are required to elucidate the underlying mechanisms.

\section{Supporting Information}

Table S1 Statistical analysis of stress hormone and inflammatory marker values in SAP and ACS patients (mean \pm SD, p-values). The table contains the results of the detailed statistical analysis: significancy values of intra- and intergroup differences ( $p$ values of intragroup/within-group comparisons for repeated measurements: pre-PGI vs post-PCI, post-PGI vs ld-PGI, and pre-PGI vs ld-PGI; separate intragroup $p$ values for SAP or ACS; overall intragroup $p$ values; and intergroup/between groups $p$ values). Significancy of the separate intragroup $p$ was a prerequisite for pairwise comparisons of the repeated measurements within each group. The $p$ value of interaction is also presented; its significancy or its relatively low value $\langle 0.3>p>0.05)$ allowed also between-group (SAP vs ACS) comparisons at each timepoint.

(DOC)

\section{Acknowledgments}

We are grateful for the devoted laboratory work of Zsuzsa Lajtos (our laboratory technician), and for the indispensible clinical help of Krisztina Hepp (chief nurse at Invasive Cardiology Unit).

\section{Author Contributions}

Conceived and designed the experiments: MK TH IF IU. Performed the experiments: MK TH IO IF GYS IU. Analyzed the data: IO GYS KB. Contributed reagents/materials/analysis tools: IF KB. Wrote the paper: MK TH IO IF GYS KB IU.

3. Matsuura E, Kobayashi K, Matsunami Y, Shen L, Quan N, et al. (2009) Autoimmunity, infectious immunity, and atherosclerosis. J Clin Immunol 29: 714721 .

4. Baetta R, Corsini A (2010) Role of polymorphonuclear neutrophils in atherosclerosis: Current state and future perspectives (Review). Atherosclerosis 210: 113 . 
5. Drechsler M, Döring Y, Megens RTA, Soehnlein O (2011) Neutrophilic ranulocytes promiscuous accelerators of atherosclerosis. Thromb Haemost 106: 839848 .

6. Soehnlein O (2012) Multiple roles for neutrophils in atherosclerosis. Circ Res 110: 875888 .

7. Weber G, Zernecke A, Libby $P$ (2008) The multifaceted contributions of leukocyte subsets to atherosclerosis: lessons from mouse models. Nature Rev Immunol 8: 802815 .

8. Adamsson Eryd S, Smith JG, Melander O, Hedblad B, Engström G (2012) Incidence of coronary events and case fatality rate in relation to blood lymphocyte and neutrophil counts. Arterioscler Thromb Vasc Biol 32: 533539

9. Avanzas P, Arroyo-Espliguero R, Gosin-Sales J, Juan Quiles, Zouridakis E, et al. (2004) Multiple complex stenoses, high neutrophil count and G-reactive protein levels in patients with chronic stable angina. Atherosclerosis 175: 151 157

10. Videm V, Wiseth R, Gunnes S, Madsen HO, Garred P (2007) Multiple inflammatory markers in patients with significant coronary artery disease. Int J Cardiol 118: 8187 .

11. Buffon A, Biasucci LM, Liuzzo G (2002) Widespread coronary inflammation in unstable angina. New Engl J Med 347: 512

12. Ott I, Neumann FJ, Gawaz M, Schmitt M, Schömig A (1996) Increased neutrophil-platelet adhesion in patients with unstable angina. Circulation 94 12391246

13. Mazor R, Schurtz-Swirski R, Farah R, Kristal B, Shapiro G, et al. (2008) Primed polymorphonuclear leukocytes constitute a possible link between inflammatio and oxidative stress in hyperlipidemic patients. Atherosclerosis 197: 937948.

14. Rosengren A, Hawken S, Ounpuu S, Sliwa K, Zubaid M, et al. (2004) Association of psychosocial risk factors with risk of acute myocardial infarction in 11119 cases and 13648 controls from 52 countries (the INTERHEART study). Lancet 364: 953962 .

15. Yusuf S, Hawken S, Ounpuu S, Dans T, Avezum A, et al. (2004) Effect of potentially modifiable risk factors associated with myocardial infarction in 52 countries (INTERHEART study): case-control study. Lancet 364: 937952

16. Kölner V, Bernardy $\mathrm{K}$ (2006) How to reduce stress and anxiety in patients undergoing catheterization? Clin Res Cardiol 95: 511513.

17. Ellard DR, Gastle PG, Mian R (2001) The effect of a short-term mental stressor on neutrophil activation. Int J Psychophysiol 41: 93100.

18. Keresztes M, Rudisch T, Tajti J, Ocsovszki I, Gardi J (2007) Granulocyte activation in humans is modulated by psychological stress. Stress 10: 271281

19. Mian R, Shelton-Rayner G, Harkin B, Williams P (2003) http:// informahealthcare.com/action/doSearch?action $=$ runSearch\&type $=$ advanced \& result $=$ true\&prevSearch $=\% 2$ Bauthorsfield $\% 3 \mathrm{~A} \% 28 \mathrm{Harkin} \% 2 \mathrm{C}+\mathrm{Brendan} \%$ result $=$ true\&prevSearch $=\% 2 \mathrm{Bauthorsfiel} \%$ observing a fictitious stressful event: haematological changes, including circulating leukocyte activation. Stress 6: 4147

20. Chrousos GP (1995) The hypothalamic-pituitary-adrenal axis and immunemediated inflammation (Seminars of Beth Israel Hospital, Boston). New Engl J Med 332: 13511362.

21. Strickland I, Kisich K, Hauk PJ, Votterod A, Chrousos GP, et al. (2001) High constitutive glucocorticoid receptor beta in human neutrophils enables them to reduce their spontaneous rate of cell death in response to corticosteroids. J Exp Med 193: 585593

22. Alevizaki M, Gimponeriu A, Lekakis J (2007) High anticipatory stress plasma cortisol levels and sensitivity to glucocorticoids predict severity of coronary artery disease in subjects undergoing coronary angiography. Metabolism 56: 222226

23. Troxler RG, Sprague EA, Albanese RA, Fuchs R, Thompson AJ (1977) The association of elevated plasma cortisol and early atherosclerosis as demonstrated by coronary angiography. Atherosclerosis 26: 151162.

24. Hamer M, Endrighi R, Venuraju SM, Lahiri A, Steptoe A (2012) Cortisol responses to mental stress and the progression of coronary artery calcification in healthy men and women. PLOS ONE 7: e31356. doi:10.1371/journal. pone. 0031356 .

25. Joynt KE, Whellan DJ, O'Gonnor CM (2003) Depression and cardiovascular disease: Mechanisms of interaction. Biol Psychiatry 54: 248261.

26. Woods A, Brull DJ, Humphries SE, Montgomery HE (2000) Genetics of inflammation and risk of coronary artery disease: the central role of interleukin6. Eur Heart J 21: 15741583

27. Gaixeta AM, Brito FS, Costa MA, Serrano CV, Petriz JL, et al. (2007) Enhanced inflammatory response to coronary stenting marks the development of clinically relevant restenosis. Catheter Cardiovasc Interv 69: 500507.
28. Gach O, Biémar G, Nys M, Deby-Dupont G, Chapelle JP, et al. (2005) Early release of neutrophil markers of activation after direct stenting in patients with unstable angina. Coron Art Dis 16: 5965 .

29. Antonsen S, Wiggers P, Dalhoj J, Blaabjerg O (1993) An enzyme-linked immunosorbent assay for plasma-lactoferrin. Scand J Clin Lab Invest 53: 133

30. Wilson MB, Nakane PK (1987) Recent development in the periodate method of conjugating horseradish peroxidase. In: Knapp W, Holubar K, Wick G, editors. Immunfluorescence and related staining techniques. Amsterdam: Elsevier. 215 224

31. Afeltra A, Caccavo D, Ferri GM, Addessi MA, de Rosa FG, et al. (1997) Expression of lactoferrin on human granulocytes: analysis with polyclonal and monoclonal antibodies. Clin Exp Immunol 109: 279285

32. Boxer LA, Haak RA, Yang HH, Wolach JB, Whitcomb JA, et al. (1982) Membrane-bound lactoferrin alters the surface properties of polymorphonuclear leukocytes. J Glin Invest 70: 10491057

33. Swain SD, Jutila KL, Quinn MT (2000) Cell-surface lactoferrin as a marker for degranulation of specific granules in bovine neutrophils. Am J Vet Res 61: 29

34. Nijm J, Kristenson M, Olsson AG, Jonasson L (2007) Impaired cortisol response to acute stressors in patients with coronary disease. Implications for inflammatory activity. J Int Med 262:375 384

35. Cocks RA, Ghan TYF, Rainer T (1998) Leukocyte L-selectin is up-regulated after mechanical trauma in adults. J Trauma (Injury Infect Crit Ciare) 45: 16

36. Suzuki H, Yokomizo S, Wakamoto S, Watanabe K, Hirose K, et al. (2000) Translocation of $\operatorname{sLE}(x)$ on the azurophilic granule membrane to the plasma membrane in activated human neutrophils. J Electron Microsc (Tokyo) 49: 359 370.

37. Bennett RM, Kokocinski T (1979) Lactoferrin turnover in man. Clin Sci 57 453460

38. Vengen IT, Dale AG, Wiseth R, Midthjelld K, Videm V (2010) Lactoferrin is a novel predictor of fatal ischemic heart disease in diabetes mellitus type 2: Longterm follow-up of the HUNT 1 study. Atherosclerosis 212:614 620.

39. Fumaz GR, Gonzalez-Garcia M, Borras X, Munoz-Moreno JA, Perez-Alvarez $\mathrm{N}$, et al. (2012) Psychological stress is associated with high levels of IL-6 in HIVinfected individuals on effective combined antiretroviral treatment. Brain Behav Immun 26: 568572

40. Stefano GB, Smith EM (1996) Adrenocorticotropin a central trigger in immune responsiveness: tonal inhibition of immune activation. Med Hypotheses 46: 471478 .

41. Strausbaugh HJ, Rosen SD (2001) A potential role for annexin 1 as a physiologic mediator of glucocorticoid-induced L-selectin shedding from myeloid cells. J Immunol 166: 6294 6300.

42. Fantidis P (2010) The role of the stress-related anti-inflammatory hormones ACTH and cortisol in atherosclerosis. Curr Vasc Pharmacol 8: 517525.

43. Walker BR (2007) Glucocorticoids and cardiovascular disease (Review) Eur J Endocrinol 157: 545559

44. Sagiyama K, Tsuchida M, Kawamura H, Wang S, Li G, et al. (2004) Agerelated bias in function of natural killer $\mathrm{T}$ cells and granulocytes after stress: reciprocal association of steroid hormones and sympathetic nerves. Clin Exp Immunol 135: 5663

45. Kopprasch S, Pietzsch J, Graessler J (2004) The protective effects of HDL and it constituents against neutrophil respiratory burst activation by hypochloriteoxidized LDL. Mol Cell Biochem 258: 121127

46. Carr AG, Frei B (2002) Human neutrophils oxidize low-density lipoprotein by a hypochlorous acid-dependent mechanism: the role of vitamin G. Biol Chem 383 627636

47. Quinn K, Henriques M, Parker T, Slutsky AS, Zhang H (2008) Human neutrophil peptides: a novel potential mediator of inflammatory cardiovascular diseases. Am J Physiol Heart Girc Physiol 295: H1817 H1824.

48. Praven EP, Sahoo JP, Kulshreshtha B, Khurana ML, Gupta N, et al. (2011) Morning cortisol is lower in obese individuals with normal glucose tolerance. Diabetes Metab Syndr Obes 4: 347352.

49. Kazakou P, Kyriazopoulou V, Michalaki M, Ierodiakonou V, Psyrogiannis A, et al. (2012) Activated hypothalamic pituitary adrenal axis in patients with metabolic syndrome. Horm Metab Res 44: 839844 
II. 


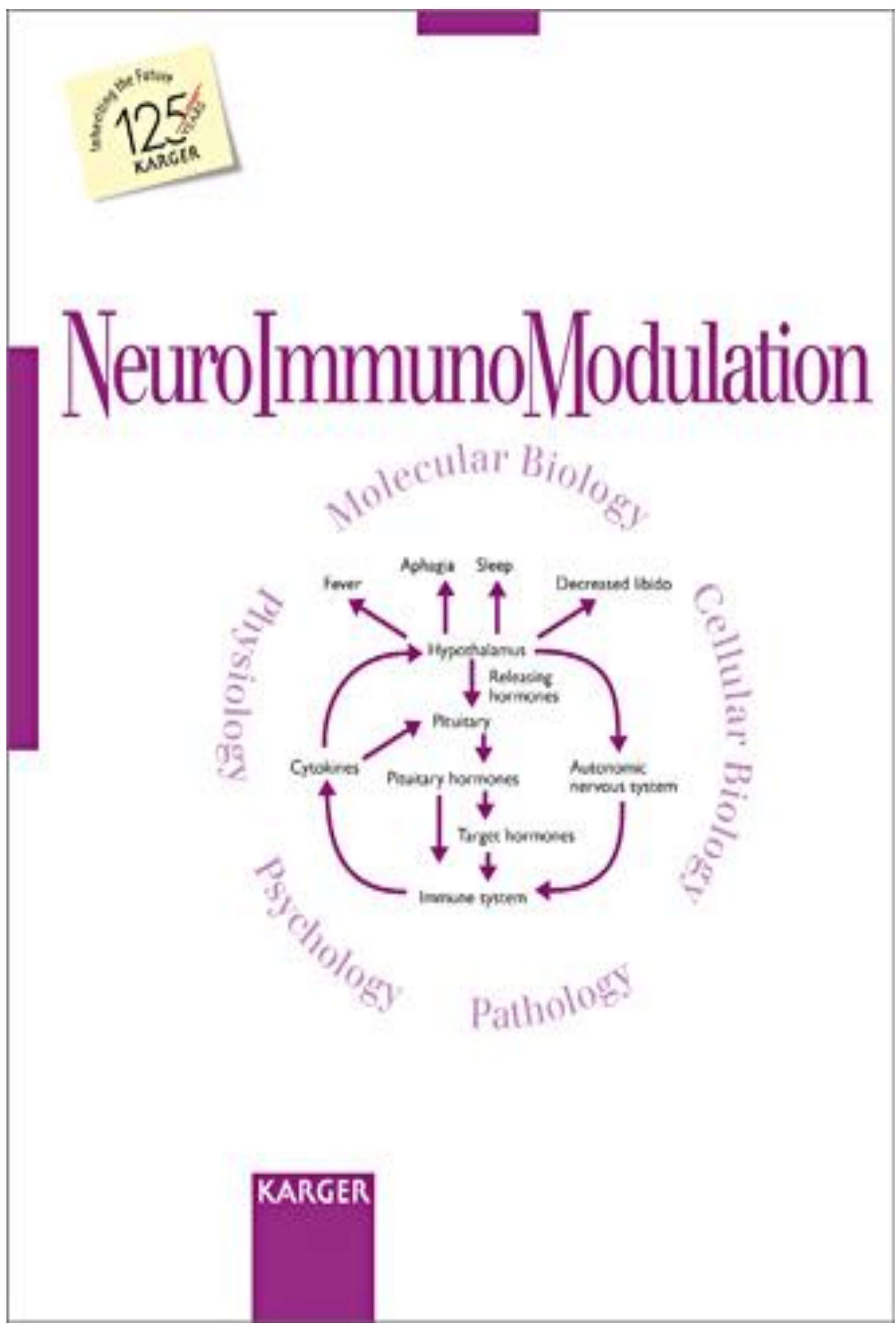




\title{
The Monocyte-to-Lymphocyte Ratio Correlates with Psycho-Neuro-Inflammatory Factors in Patients with Stable Coronary Artery Disease
}

\author{
Gyöngyi Serfózóa Tamás Horváthc Imre Földesi ${ }^{b}$ Beatrix Rafaeld \\ Roland von Kănele, $f$ Margit Keresztes ${ }^{a}$
}

Departments of 'Biochemistry and 'Laboratory Medicine, and Imvasive Cardiology Unit, Centre of Cardiology, Medical Faculty, and ¿Department of Psychology, Faculty of Humanities, University of Szeged, Szeged, Hungary; -Department of Neurology, Bern University Hospital, Bern, and 'Department of Psychosomatic Medicine, Barmelweid Clinic, Barmelweid, Switzerland

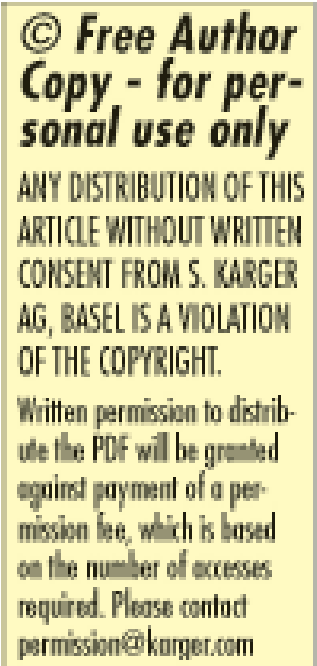

\section{Key Words \\ Atherosclerosis - Cardiovascular disease - Depressive symptoms - Inflammation - Monocyte-to-lymphocyte ratio . Psychological stress · Psychoneuroendocrinology · Social support · Stable coronary artery disease}

\begin{abstract}
Background: Psychosocial stress and depression have been recognized as major risk factors of coronary artery disease (CAD). Although monocytes are known to be key players in atherosclerosis, monocyte-based associations with psychoneurœendocrino-immuno-inflammatory (PNI) markers have not been widely investigated in stable CAD. Objective: We examined associations between the monocyte-to-lymphocyte ratio (MLR) and key PNI markers in stableCAD. Methods: We studied 23 patients with stable CAD who completed the Beck Depression Inventory (BDI) and Rahe's Brief Stress and Coping Inventory. A white blood cell differential was performed, and levels of cortisol, chromogranin A (CgA), LL-37, interleukin-6 (IL-6) and C-reactive protein (CRP) were assayed in plasma. Results: Monocyte fraction, MLR and plasma CgA levels exceeded reference values, the social support score was low, and 7 patients had elevated BDI scores. In the multivariate-adjusted analysis, a higher MLR was associated
\end{abstract}

\section{KARGER}

E-Mail kargenelargarcom

www.kangsicoom/nim with greater depressive symptom severity $(r=0.624, p<$ $0.01)$ as well as with higher concentrations of $\mathrm{CgA}(r=0.660$, $p<0.01)$, LL-37 $(r=0.643, p<0.01), \mathrm{IL}-6(r=0.532, p<0.05)$ and $C R P(r=0.470, p<0.05)$. BDl scores associated with $C g A$ concentration $(r=0.618, p<0.01)$ and $\mathrm{CgA}$ level correlated negatively with the social support score $(r=-0.511, p<0.05)$. Concluslons: Our findings suggest that, in patients with stable CAD, elevated MLR may be associated with depressive symptoms, with increased neurcend ocrine-sympathetic activity (marked by $\mathrm{CgA}$ ) and inflammatory markers that are pertinent to atherosclerosis initiation and progression. The increased neuroendocrine-sympathetic activity correlated with low social support and depressive symptom severity. The MLR might serve as an easy-to-obtain and inexpensive proxy measure of an activated PNI network in stable CAD.

- 2016 S. Karger AG, Basol

\section{Introduction}

Psychological stress and depression are increasingly recognized as major risk factors for the incidence of coronary artery disease (CAD) as well as independent predictors of poor cardiac prognosis [1-5]. Stress has been suggested to lead to both depression and CAD [2]. While

\section{- 2016 S. Kargor AG, Basel \\ Margit Xeresctes} 1021-7401/16/0000-0000839.50/0
Department of Biochemitry, Modral Faoulty

Universtity of Siaged, Dam ter 9

HU -6720 szogad (Hungary)

E-Mall harestas margrepmadu-sagad.hu 
soctal isolation and a lack of social support are also risk factors for CAD, low 'meaning of life' (life meaning) scores might increase the risk of CAD mortality $[1,5-7]$. The sympathetic-adrenomedullary system and the hypothalamic-pitultary-adrenocortical (HPA) axis critically govern the stress response. To some extent, plasma cort1sol levels indicate HPA axis activity and plasma chromogranin A (CgA) levels reflect neuroendocrine and sympathoadrenal activity $[8,9]$.

Cytokines are key players of the psycho-neuroendocrine-/Immuno-Inflammatory (PNI) system; they exert a critical, early role in atherogenesis [1]. IL-6 is a main regulator of the systemic inflammatory response and induces the acute-phase reaction during which C-reactive protein (CRP) is synthestzed by the liver as well as being a highly potent stimulator of the HPA axis $[10,11]$. Both IL-6 and CRP are major markers of vascular inflammation in CAD [12], and their plasma concentrattons are increased in individuals with psychosocial stress and depression $[13,14]$. Another key but less investigated inflammatory marker is LL-37 that is typically produced by macrophages in atherosclerotic lesions $[15,16]$.

Both neutrophils and macrophages (mostly derived from monocytes) have fundamental roles in the formation and progression of coronary atherosclerotic plaques [17-19]. Several studtes found that high neutrophil count or neutrophil-to-lymphocyte ratlo are characteristic for coronary stenosis and could predict $\mathrm{CAD}$ and major adverse cardiac events, including myocardial infarction [20-25]. Waterhouse et al. [26], however, have shown that of all leukocyte types, the monocyte count has the strongest posittve and independent relationship with CAD risk in asymptomatic adults. In addition, the monocyte count predicted the severity of atherosclerotic stenosis and plaque progression in acute coronary syndromes $[24,27]$. In contrast, in patients with acute chest pain, a low lymphocyte count was indicattve of an increased risk of developing myocardial infarction and all-cause mortality [28]. A decreased lymphocyte-to-monocyte ratio correlated with CAD and prior myocardial infarction in addition to a high risk for critical limb ischemta [29]. As yet, only a few studies have analyzed associations between monocyte counts/fractions and psychological factors. In pattents with acute coronary syndrome, a higher monocyte fraction was found to be associated with less soctal support and more life-event stress [30].

The primary alm of our study was to investigate the association of the monocyte-to-lymphocyte ratlo (MLR) with psychosoctal factors and neuroendocrino-inflammatory markers in pattents with stable CAD. To our knowledge, such correlations have not previously been studied. Specifically, we hypothesized significant assoctathons of the ML.R with depressive symptoms, stress and coping scores as well as with the circulating levels of cortisol, CgA, IL-6, CRP and LL-37 in patlents with stable angina pectoris. A secondary alm was to perform correlation analyses of psychosoctal factors and neuroendocrine and inflammatory markers.

\section{Methods}

\section{Pattents}

Twenty-three patients with stable CAD participated in our study. For inclusion, significant stenosis of at least 2 major coronary arterles was to be demonstrated by coronary anglography (stgnificant diameter stenosis $>50 \%$ ). Excluston criterla included bypass surgery, cardiogenic shock, malignancy (e.g. prostate cancer), any immunological disorders (including rheumatold arthritts and chronic atrophic gastritis type A) or acute inflammations, immunosuppressive/anti-inflammatory medication (except lowdose aspirin), recent major trauma/surgery, impaired renal functhon and drug/alcohol abuse. The severity of heart fatlure symptoms was graded according to the New York Heart Assoclation (NYHA) functional classiflcation (grades I-IV).

Partictpants provided written consent for conducting the study protocol which was approved by the Medical Ethics Committee at our Medical Faculty (Human Investigation Revlew Board, ref. No. $49 / \mathrm{B}-125 / 2008,2382$ ). The investigation conformed to the princtples of the Declaration of Helstnkt.

\section{Psychosocial Measures}

We measured the severtity of depressive symptoms with the 21item Beck Depression Inventory (BDI) [31], using its Hungarian version [32]. Participants rate every item on a scale 0 to 3 . Total scores indicate no clinically relevant depresstve symptoms (0-9), mild (10-18), moderate (19-25) and severe (25-63) depressive symptoms.

To assess everyday stress level and coping capactty, we applied Rahe's Brief Stress and Coping Inventory (BSCI, a multidimenslonal self-describing device) [33]. To obtain a global stress-coping score, total stress score was subtracted from total coping score. Score evaluation was performed according to the validated Hungarlan verston: worrisome/non-sufficient $(-15$ to -5$)$, sufficlent $(-4$ to -1$)$, good ( 0 to 4$)$ or very good (5 to 15) [34]. Both the stress and coping scales are divided into 5 subscales. Coping subscales measure health habits, social support, responses to stress, life sattsfaction and 'purpose and connection'. Spectifcally, the soctal support subscale measures the degree of a participant's soctal network. The purpose and connection subscale (also called meaning of/in life or 'life meaning') reflects to what extent subjects feel their life is 'worth living' [7]. Subscale scores range from 0 to 14 (social support) or 0 to 16 (life meaning), and coping with stress is indicated as worrtsome/non-sufficlent (0-7), sufficient (8-10), good (1113) or very good/excellent (14-16). Neuroimmunomodulation
DOI: $10.1159 / 000443835$
Serfözó//Horváth/Földest/Rafael/ von Känel/Keresztes 


\section{Blood Sampling and Laboratory Assays}

Fasting venous blood samples were collected 1 day before coronary anglography between 7 and $10 \mathrm{a} . \mathrm{m}$. Blood counts, total cholesterol, triglycerides, high-density lipoprotein (HDL)-cholesterol and high-sensittvity (hs)-CRP were determined in automatic analyzers and/or using commercial kits (Roche); low-denstty lipoprotein (L.DL)-cholesterol was calculated according to the Friedewald formula. Since no reference interval was avallable for the ML.R, we used the ratio of the median values of the monocyte and lymphocyte reference ranges (counts) as a reference: 0.19 .

For the assessment of plasma levels of $\mathrm{CgA}$, cortisol, LL-37 and IL-6, we collected blood into cooled $\left(4^{\circ} \mathrm{C}\right)$ EDTA-Vacutainer tubes. CgA was assayed with a radiolmmunoassay kit (CGA-RIA CT, CIS Bto International, Gif-sur-Yvette, France; intra- and interassay CVs: $<7 \%$ ). Cortisol was determined by radiotmmunoassay (DSL-2100, Dlagnostic Systems Laboratories, Webster, Tex., USA; intra- and interassay CVs: $<12 \%$ ). LL- 37 concentration was measured with an ELISA kit (HK321, Hycult Biotech Inc., Uden, The Netherlands; intra- and interassay CVs: $<10 \%)$. Due to technical problems, LL-37, corttsol and L.DL-cholesterol values were not avatlable for 1 patient. IL-6 was assayed using a high-sensttivity ELISA kit (BMS213HS; Bender MedSystems GmbH, Vienna, Austria; intra- and interassay CVs: 4.95 and $6.0 \%$, respectively).

\section{Statistical Analysis}

We first calculated the necessary sample size to achieve clinically meaningful correlation coefflclents of $r \geq 0.6$ between var1ables of interest with a power of $80 \%$ and a $5 \%$ level of signiflcance. The power analysts ytelded a sample size of 19 patients, and so we recrulted 24 patients in order to compensate for potenttal dropouts. In fact, 1 pattent was transferred to a county hospital before being assessed, leaving 23 participants for our study.

Cohen [35] provides guldelines for interpreting the effect size of correlation coefficlents ( $r$ ) with values of $0.1,0.3$ and 0.5 as small, medium and large, respectively. Therefore, regardless of $p$ values, correlation coeffictents $\geq 0.6$ may yteld spurious findings, even when performing multiple tests. Spectfically, we did not adjust p values for multtple compartsons because of our pre-established primary hypothesis that MLR would show significant assoclattons with psychosoctal factors and neuroendocrine and inflammation markers. In this still-nascent fleld of research, the Bonferronl adjustment bears the risk of deeming truly important differences non-significant [36]. Secondary hypotheses were formulated for significant assoclations amongst psychosocial factors and neuroendocrine and inflammation markers (but not MLR) [37].

All statistical analyses were performed using IBM-SPSS software, v20.0 (SPSS Inc., Chicago, III., USA) with a two-sided significance level of $\mathrm{p}<0.05$. For continuous variables, values are presented as mean \pm standard devlation (SD). Data that did not show a normal distribution (Le. MLR, CRP and BDI) were $\log _{10} 0^{-}$ transformed before analysts. The Student $t$ test was applied to test for differences in psychosoclal factors and neuroendocrine and inflammatory markers according to sex, dlabetes smoking and medications.

To examine assoctations of MLR with psychosoctal factors and neurcendocrine and inflammatory markers, we employed the bivariate and multivarlate Pearson correlation analyses with adjustment for age, sex and BMI. Stmilar analyses were performed to estimate the correlations amongst psychosoclal factors and neuro- endocrine and inflammatory markers. As proton-pump inhibitors could elevate plasma $\mathrm{CgA}$, and $\mathrm{CgA}$ levels could also be elevated in chronic heart fallure, we additionally corrected $\mathrm{CgA}$ models for intake of this medication and NYHA grades [38, 39]. As sedative use showed a stgnificant relation with BDI scores, we also controlled for this in the models with the BDI.

\section{Results}

\section{Pattent Characteristics}

The study particlpants were 18 men and 5 women. The mean age of the sample was $62.9 \pm 10.6$ years (range 43-79 years). Regarding established cardiovascular risk factors, our pattents were, on average, overweight, with a BMI of $28.8 \pm 4.0$. Elght (35\%) had diabetes mellitus and $2(9 \%)$ were current smokers. There were 3 (13\%), 4 (17\%) and $5(22 \%)$ pattents in NYHA groups I, II and III, respectively. All of the pattents took aspirin, and anthypertenstves and statins were taken by $22(96 \%)$ and $21(91 \%)$, respectively. Moreover, 16 (70\%) were on proton-pump Inhibitors and $9(39 \%)$ on sedattves. No patlents were on antidepressants or $\mathrm{H}_{2}$-receptor blockers.

\section{Laboratory Parameters: MLR and Neuroendocrine and Inflammatory Factors}

As can be seen in table 1, the monocyte fraction $(8.68 \%$, SD 2.97) exceeded the maximal reference value (7\%), and monocyte count approached the upper reference limit, while the lymphocyte fraction was close to the minimal reference value. Thus, the MLR ylelded a relattvely high value of 0.32 (SD 0.14 ), exceeding the predefined reference value of 0.19 . Normal values were observed for the other white blood cells (shown: total leukocyte count, neutrophil count and fraction) and blood lipids, except for slightly elevated triglyceride levels. Of the neuroendocrine and inflammatory parameters, only CgA was outside the reference range, exceeding the upper reference value as per the recent literature [40]. CRP was close to its upper reference limit.

\section{Psychosocial Factors}

While the average depressive symptom level of our participants was in the subclinical range, 7 (30\%) had BDI scores that indicated clinically relevant depressive symptoms, 1.e. 5 with mild symptoms, 1 with moderate symptoms and 1 with severe symptoms. Nonetheless, a subgroup analysis showed significantly higher BDI scores in patlents on sedatives versus those not on prescribed sedathes $(12.8 \pm 12.6$ vs. $3.9 \pm 4.4 ; p<0.05)$. 
Table 1. Characteristics of the 23 patients with stable CAD

\begin{tabular}{llcc}
\hline & Parameter & Value & Ref. range \\
\hline Leukocyte panel & total leukocyte count, G/1 & $6.73 \pm 1.22$ & $3.7-9.5$ \\
& neutrophil granulocyte count, G/1 & $4.05 \pm 1.04$ & $1.7-6.1$ \\
& neutrophil granulocyte fraction, \% & $59.70 \pm 6.86$ & $44-68$ \\
& monocyte count, G/l & $0.58 \pm 0.23$ & $0.2-0.6$ \\
& monocyte fraction, \% & $8.68 \pm 2.97$ & $5-7$ \\
& lymphocyte count, G/l & $1.90 \pm 0.41$ & $1.0-3.2$ \\
& lymphocyte fraction, \% & $28.82 \pm 6.30$ & $27-34$ \\
& MLR & $0.32 \pm 0.14$ & $0.19^{5}$ \\
\hline L.ipld panel & total cholesterol, mmol/1 & $4.30 \pm 1.35$ & $<5.2$ \\
& HDL-cholesterol, mmol/1 & $1.18 \pm 0.32$ & $>1.0$ \\
& LDL-cholesterol, mmol/1 & $2.27 \pm 0.94$ & $<3.0$ \\
& triglycerides, mmol/1 & $2.04 \pm 1.25^{\mathrm{a}}$ & $<2.0$ \\
\hline Neuroendocrine markers & cortisol, nmol// & $294.45 \pm 88.59$ & $160-620$ \\
& CgA, ng/ml & $178.81 \pm 105.50^{2}$ & $23-153$ \\
\hline Inflammatory markers & L.L-37, ng/ml & $55.31 \pm 12.44$ & $25-250$ \\
& IL-6, pg/ml & $1.83 \pm 1.35$ & $<8.7$ \\
& CRP, mg/l & $4.93 \pm 5.27$ & $<5.0$ \\
\hline Psychosoctal scores & BDI & $7.4 \pm 9.4$ & see Methods \\
& BSCI & $-0.7 \pm 4.2$ & see Methods \\
& Global stress-coping & $7.9 \pm 2.4$ & see Methods \\
& Social support & $11.9 \pm 1.9$ & see Methods \\
\hline & Purpose and connection & &
\end{tabular}

Varlables are means \pm SD. There were 22 patients in the sample for the LDL-cholesterol, cortisol and LL-37 measurements. ${ }^{2}$ Values greater than the maximal reference range. ${ }^{b}$ Here we used the ratio of the median values of the monocyte and lymphocyte reference ranges (counts).

The mean global stress-coping score was below zero, reflecting higher levels of stress than the coping scores, but in the 'sufficient' range. The relattvely low mean social support score was close to the 'sufficlent' range, and the life meaning score was evaluated as 'good'.

Correlattons between the MLR and Psychosoctal

Factors, Neuroendocrine and Inflammatory

Parameters

Bivarlate Analysis

Table 2 shows that patients with higher MLR values had significantly higher concentrations of CgA, LL-37, IL-6 and CRP (all p values $<0.03$ ), showing mostly large effects.

\section{Multtvarlate Analysis}

After controlling for age, sex and BMI (and additionally for proton-pump inhibitor intake and NYHA grade for $\mathrm{CgA}$ ), the significance and effect sizes of the associations between the MLR and CgA and LL-37 considerably
Increased ( $r>0.6, p=0.003$, power $>0.95)$; in contrast, in the case of IL-6 and CRP, these values decreased or were not remarkably altered $(r>0.45, p<0.04$, power $>0.65$ ). With additional adjustment for sedattve use, higher MLR was assoclated with greater depresslve symptom severity, showing a large effect $(r>0.6, p=0.004$, power $>0.95)$. Similarly to the bivariate analysis, plasma cortisol and the 2 elected coping subscales (1.e. soctal support and purpose and connection) did not show a significant correlation with the MLR. For illustrattve purposes, the significant multivariate-adjusted correlations between the MLR and PNI markers are shown in the online supplementary figures (see www.karger.com/dot/10.1159/000443835 for all online suppl. material).

\section{Correlations amongst Psychosoctal Factors and}

Neuroendocrine and Inflammatory Markers

As can be seen in table 3, significant and large effects were observed for the association between lower soctal support scores and higher $\mathrm{CgA}$ levels and between lower 
Table 2. Correlations between the ML.R and PNI system markers in 23 stable CAD pattents

\begin{tabular}{|c|c|c|c|c|c|c|}
\hline & \multicolumn{3}{|c|}{ Btvariate correlations } & \multicolumn{3}{|c|}{ Multivariate correlations } \\
\hline & $\mathbf{r}$ & $\mathrm{p}$ & power & r & $\mathrm{p}$ & power \\
\hline Cortisol & 0.279 & n.s. & 0.254 & 0.277 & n.s. & 0.251 \\
\hline $\mathrm{CgA}$ & $0.462^{+}$ & 0.026 & 0.664 & $0.660^{+*}$ & 0.003 & 0.980 \\
\hline LL. -37 & $0.537^{+*}$ & 0.010 & 0.811 & $0.643^{+*}$ & 0.003 & 0.963 \\
\hline IL -6 & $0.620^{+4}$ & 0.002 & 0.951 & $0.532^{+}$ & 0.016 & 0.819 \\
\hline CRP & $0.474^{+}$ & 0.022 & 0.692 & $0.470^{+}$ & 0.036 & 0.683 \\
\hline BDI & 0.216 & n.s. & 0.173 & $0.624^{+*}$ & 0.004 & 0.954 \\
\hline BSCI social support & 0.221 & n.s. & 0.180 & -0.260 & n.s. & 0.234 \\
\hline BSCI life meaning & -0.124 & n.s. & 0.088 & -0.084 & n.s. & 0.067 \\
\hline
\end{tabular}

In the multivartate analysts, all parameters were adjusted for age, BMI and sex. Additional adjustments were: intake of proton-pump inhtbitors and grades of heart fallure $(\mathrm{CgA})$ and intake of sedattves (BDI). There were 22 pattents in the sample for the cortisol and LL -37 measurements. $\mathrm{r}=$ Pearson's correlation coefficlent; $\mathrm{n} . \mathrm{s}$. = not stgnificant at the level of $a=0.05 .{ }^{*} p<0.05,{ }^{*+} p \leq 0.01$.

Table 3. Correlations amongst psychological/psychosoctal factors and neuroendocrine and inflammatory markers in 23 stable CAD patlents

\begin{tabular}{|c|c|c|c|c|c|c|}
\hline & \multicolumn{3}{|c|}{ Bivarlate correlations } & \multicolumn{3}{|c|}{ Multivariate correlations } \\
\hline & $\mathrm{r}$ & $\mathrm{p}$ & power & $\mathbf{r}$ & $\mathrm{p}$ & power \\
\hline $\mathrm{BDI}-\mathrm{CgA}$ & 0.275 & n.s. & 0.258 & $0.618^{*+4}$ & 0.008 & 0.949 \\
\hline BDI - LL-37 & 0.380 & n.s. & 0.45 & $0.502^{*}$ & 0.034 & 0.736 \\
\hline BSCI social support - $\mathrm{CgA}$ & $-0.502^{+}$ & 0.015 & 0.756 & $-0.511^{*}$ & 0.030 & 0.776 \\
\hline BSCI life meaning - CRP & $-0.555^{*+}$ & 0.006 & 0.862 & $-0.467^{*}$ & 0.038 & 0.676 \\
\hline
\end{tabular}

In the multtvarlate analysis, all parameters were adjusted for age, BMI and sex. Additional adjustments were: intake of proton-pump inhibitors and grades of heart failure $(\mathrm{CgA})$ and intake of sedattves (BDI). $\mathrm{r}=$ Pearson's correlation coefficient; $\mathrm{n} . \mathrm{s}$. $=$ not stgnificant at the level of $\mathrm{a}=0.05 .{ }^{*} \mathrm{p}<0.05{ }_{\mathrm{v}}{ }^{*+} \mathrm{p}<0.01$

life meaning scores and higher CRP levels, both in the b1varlate and multtvarlate analysts $(\mathrm{r}<-0.45$, all $\mathrm{p}$ values $<0.04$, power $>0.65$ ). In addition, more severe depressive symptoms significantly correlated with higher LL-37 levels $(r>0.5, p<0.04$, power $>0.7)$ and particularly with elevated $\mathrm{CgA}$ concentrations $(\mathrm{r}>0.6, \mathrm{p}<0.01$, power $>0.9$ ) in the multivarlate analysis.

\section{Discussion}

The main finding of our study is that an elevated MLR was assoclated with depressive symptoms and increased neuroendocrine-sympathetic activity (marked by $\mathrm{CgA}$ ) in pattents with stable CAD. Furthermore, enhanced neuroendocrine-sympathetic activity correlated with elevat- ed depressive symptom levels and decreased soctal support.

We showed an increased monocyte fraction and elevated MLR in our stable CAD patients, reflecting an inflammatory state. In support of this, the MLR correlated with circulating levels of LL-37, IL-6 and CRP. Surprisingly, the reclprocal of the pattents' MLR value $(1 / 0.32=$ 3.125) is almost equal to the crittcal lymphocyte-tomonocyte ratio of 3.1 that has been found in patients with a high risk of severe (atherosclerotic) limb ischemia, which by itself is frequently associated with CAD [29].

The increased plasma CgA levels could indicate general neuroendocrine overactlvity including elevated sympathoadrenal activity $[41,42]$; importantly, we controlled for the myocardial release of CgA (in cardiac pressure/ volume overload) and the effect of proton-pump inhibl- 
tors. Recent research with $\mathrm{ApoE}-\mathrm{f}^{-}$mice shows heightened sympathetic activity to be directly related to monocytosis via the release of hematopolettc cells from the bone marrow (medtated by norepinephrine and $\beta 3$ adrenergic receptors), followed by increased monocytopolesis in the spleen [43]. Monocyte delivery to the bloodstream could result in the progression of atherosclerosis through the kindling of inflammation, particularly in the wake of myocardial infarction $[43,44]$. Similarly, elevated plasma CgA could indicate sympathetic activation capable of inducing blood monocytosis. This might help to explain the significant correlation between CgA and MLR, showing a large effect. In turn, activated monocytes/macrophages may also release chromograntns [45].

About $30 \%$ of our patients had clinically relevant levels of depressive symptoms, and the global stress-coping score was, on average, 'suffictent' only, thereby suggesting that our sample endorsed limited coping skills under stress. The more severely depressed our patlents were, the higher were their CgA plasma concentrations, suggesting that increased activity of the neuroendocrino-sympathoadrenal system accompantes depressive symptomatology. This corresponds to prevlous studies in individuals with depression and chronic stress who showed sympathoadrenal hyperactivity, which, further downstream, could lead to the activation of monocytes/macrophages vla $\beta$-adrenergic receptors, as has been shown in CAD $[4$, 46].

Furthermore, proinflammatory cytokines (especially IL-1) secreted by actlvated monocytes/macrophages could underlie the link between the MLR and depressive symptoms in our study; prolnflammatory cytokines facilitate the development of depressive mood by interferIng with neurotransmitter functioning $[47,48]$. Associations of MLR with IL- 6 and CRP support the importance of activated monocytes in the production of IL- 6 , the most important inducer of CRP synthesis and acutephase response, in general [11]. Notably, IL-6 and CRP are proposed to be major factors linking stress/depression and $\mathrm{CAD}[2,11,46]$. According to a recent study, CAD patients with depression exhibit significantly higher CRP and lower plasma cortisol levels than nondepressed CAD patients [49]. Similarly, our patients were also characterized by relattvely high CRP values and relattvely low plasma corttsol, perhaps a consequence of long-term negattve feedback in chronic stress $[49,50]$.

In our study, depressive symptoms were significantly assoclated with LL-37 which is also deemed to be an inflammatory marker; this supports the notion of a possible link between inflammation and depression [14]. In fact,
LL-37 exerts a multtude of antimicrobial and immunomodulatory effects, along with playing a role in apoptosis and wound healing; it is expressed widely by different leukocytes [51]. Since monocytes and related macrophages in atherosclerotic lesions abundantly release LL-37, the observed correlation of MLR with LL-37 in stable CAD is not surprising $[15,16]$.

The correlation of the low social support score with Increased $\mathrm{CgA}$ presumably indicates that elevated sympathoadrenal activity could be a consequence of psychosoclal stress that may be due to limited emotional/soctal support [52]. Friedler et al. [53] propose (mainly on the basis of investigations using rodent models) that the increased sympathetic activity related to chronic psychosoclal stress (like soctal tsolation) could drive hematopolests towards the formation of monocytic/granulocytic progenitor cells in bone marrow through $\beta$-adrenergic receptor-mediated signaling and altered gene expression. This Idea complements the above-mentioned mechantsm of monocytosis $[43,44]$.

Our study has several limitations, foremost being the small sample stze and the cross-sectional design, preventing causal inferences. However, the effect stzes of the statistically significant correlations were large and thus support the validity of our results. To avold model overfitting in the multtvariate analysis, we were unable to control for additional covariates, namely established cardiovascular risk factors like diabetes and smoking or for medications (except for proton-pump inhibitors and sedatives in 2 models). The average depressive symptom score was in the normal range for $70 \%$ of our patients, so if our findIngs would hold in a sample of more severely depressed CAD patlents remains unclear. Due to the lack of a control group, we cannot exclude the possibility that similar associations of MLR with psychological and psychosocial factors and neuroendocrine and inflammatory markers also exist in healthy individuals. However, the elevated monocyte fraction and MLR values, the overactlvated neuroendocrine-sympathetic system (marked by increased CgA level) and the low soctal support score demonstrate pathological characteristics in our pattents.

Our findings parallel the observations according to which depressed and/or stressed patlents with an activated sympathetic nervous system are characterized by a chronIc inflammatory state that could lead to CAD and/or its progresslon $[4,46,54]$. Our results also support findings on the importance of sympathetic activity in monocytosis and in monocyte activation, and on the significance of the psychosoctal risk factors of CAD (like limited soctal support) in promoting stress. By integrating all of this reason- 
Ing, the following simplified picture of a viclous cycle/network seems to emerge: a low level of soctal support results in stress with predominant sympathetic activation, which then initiates increased monocyte count and activity, monocyte secretion of proinflammatory cytokines (with the assoctated low-grade systemic inflammation) faclltates the development of CAD and/or depression and this may further enhance the activity of the PNI network (e.g. vla chest pain and stress in angina pectoris). Although not tested here, our results suggest that psychosocial interventions targeted at, for instance, reducing chronic stress, Improving social support and alleviating depresstve symptoms, could favorably affect the vicious cycle of interacting psychosocial factors and neuroendocrine and inflammatory markers, thereby improving both the mental and physical health of CAD patients $[52,55]$.
To conclude, our study revealed that the MLR, an inexpensive and easily accessible laboratory measure, may be employed as a PNI correlate marker in the routine clinical monitoring of patients with stable CAD. Further investigations in this multidisciplinary field are required to elucldate the detalled functional connections of the PNI network in CAD that could facilitate the prevention and treatment of atherosclerosis in the future through innovattve means.

\section{Acknowledgments}

We are grateful for the devoted laboratory work of Zsuzsa Lajtos (our laboratory techniclan) and the indispensable clinical help of Krisztina Hepp (chlef nurse at Invastve Cardiology Unit).

\section{References}

1 Everson-Rose SA, Lewis TT: Psychosocialfactors and cardiovascular diseases. Annu Rev Public Health 2005:26:469-500.

2 Joynt KE, Whellan DJ, O'Connor CM: Depression and cardiovascular disease: mecha. nisms of interaction. Biol Psychiatry 2003;54: 248-261.

3 Lichtman JH, Bigger JT, Blumenthal JA, Frasure-Smith N, Kaufmann PG, Lespérance F, Mark DB, Sheps DS, Taylor CB, Sivarajan Froelicher E: Depression and coronary heart disease (recommendations for screening, re ferral, and treatment). Circulation 2005;118: $1768-1775$.

4 Rosengren A, Hawken S, Ounpuu S, Sliwa K, Zubaid M, Almahmeed WA: Association of prychoeocial risk factors with risk of acute myocardial infurction in 11119 cases and 13,648 controls from 52 countries (the IN. TERHEART study). Lancet 2004;364:953 962

5 Steptoe A, Kivimaki M: Stress and cardiovas. cular disease: an update on current knowl. edge. Annu Rev Public Health 2013;34:337354.

6 Barth J, Schneider S, von Känel R: Lack of 90 cial support in the etiology and the prognosis of coronary heart disease: a systematic review and meta-analyzis. Psychosom Med 2010;72: 229-238.

7 Skrabski A, Kopp M, Rózsa S, Réthelyi J, Rahe RH: Life meaning an important correlate of health in the Hungarian population. Int J Behav Med 2005;12:78-85.

8 D'amico MA, Barbara Chinassi B, Irzicupo P. Manzoli L, Di Baldassarre A: Biological func tion and clinical relevance of chromogranin $A$ and derived peptides. Endocr Connect 2014; 3:R45-R54.
9 Taupenot L, Harper KI, O'Cannor D: The chromogranin-secretogranin family. N Engl] Med 2003:348:1134-1149.

10 Chrousos GP: The hypothalamic-pituitaryadrenal axis and immune-mediated inflammation. N Engl J Med 1995;332:1351-1362.

11 Woods A, Brull DI, Humphries SE, Montgomery HE: Genetics of inflammation and risk of coronary artery disease the central role of interleukin-6 (review). Bur Heart J 2000;21: 1574-1583.

12 Ramachandran SV: Biomarkers of cardiovas. cular disease: molecular basis and practical considerations. Circulation 2006;113:23352362.

13 Keecolt-Glaser JK, Gouin JP, Hantsoo L-Close relationships, inflammation, and health. Neurosci Biobehav Rev 2010;35:33-38.

14 Stewart JC, Rand KI, Muldoon MP, Kamarck TW: Aspective evaluation of the directionality of the depression-inflammation relationship. Brain Behav Immun 2009;23:936-944.

15 Ciarnei CD, Tapper H, Bjartell A, Sternby $\mathrm{NH}$, Bodelsson M: Human antimicrobial peptide L.L.37 is present in atherosclerotic plaques and induces death of vascular smooth muscle cells: a laboratory study. BMC Cardiovasc Disord 2006;6ce49.

16 Edfeldt K, Agerberth B, Rottenberg ME, Gudmundsson GH, Wang XB, Mandal K, Xu Q Yan ZQ: Involvernent of the antimicrobial peptide LL-37 in human atherosclerosis. Arterioscler Thromb Vasc Biol 2006;26:15511557.

17 Molawi K, Wolf Y, Kandalla PK, Favret J, Hagemeyer N, Freniel K, Pinto AR, Klapproth $\mathrm{K}$, Henri S, Malissen B, Rodewald H-R, Rosenthal NA, Bajenoff M, Prinz M, Jung S, Sieweke MH: Progressive replacement of em- bryo-derived cardiac macrophages with age. I Exp Med 2014:211:2151-2158.

18 Ross R: Atherosclerosis - an inflammatory disease. N Engl J Med 1999;340:115-126.

19 Weber C. Noels H: Atherosclerveis: current pathogenesis and therapeutic options. Nat Med 2011;17:1410-1422

20 Adamsson Eryd S, Smith JG, Melander O. Hedblad B, Engstrōm G: Incidence of coronary events and case fatality rate in relation to blood lymphocyte and neutrophil counts. Arterioeder Throenb Vase Biol 2012:32:533 539.

21 Avanzas P, Arroyo-Espliguero R, Cosin-Sales J, Quiles J, Zouridakis E, Kaski JC: Multiple complex stenoses, high neutrophil count and C-reactive protein levels in patients with chronic stable angina. Atherosclerosis 2004; 175:151-157.

$22 \mathrm{HeJ}$, Li J, Wang Y, Hao P, Hua Q: Neutrophil. to-lymphocyte ratio (NLR) predicts mortality and adverse-putcomes after ST-segment elevation myocardial infarction in Chinese people. Int J Clin Exp Pathol 2014:7:4045-4056.

23 Home BD, Anderson IL, John IM, Weaver A, Bair TL Jensen KR, Renlund DG, Muhlestein JB: Which white blood cell subtypes predict increased cardiovascular risk? J Am Coll Cardiol 2005;45:1638-1643.

24 Huang G, Zhong XN, Zhong B, Chen YQ, Liu ZZ, Su L, Ling Z-Y, Cao H, Yin Y-H: Sugnificance of white blood cell count and its subtypes in patients with acute coronary syndrome. Eur J Clin Invest 2009;39:348-358.

25 Papa A, Emdin M, Passino C, Michelassi C, Battaglia D, Cocci F: Predictive value of elevated neutrophil-hymphocyte ratio on cardiac mortality in patients with stable coronary artery disease. Clin Chim Acta 2008;395:27-31. 
26 Waterhouse DF, Cahill RA, Sheehan F, McCreery CI: Prediction of calculated future cardiovascuhr disease by monocyte count in asymptomatic population. Vasc Health Risk Manag 2008;4:177-187.

27 Nozawa N, Hibi K, Endo M, Sugano T, Ebina T, Kosuge M, Tsukahara K, Okuda J, Umemura S, Kimura K: Association between circulating monocytes and coronary plaque progression in patients with acute myocardial infaretion. Circ J 2010;74:1384-1391.

28 Núnex J, Sanchis J, Bodi V, Núnez E, Mainar L, Heatta AM, Husser O, Minana G, Merlos P, Darmafil H, Pellicer M, Lläcer A: Relationship between low hymphocyte count and major cardiac events in patients with acute chest pain, a nan-diagnostic electrocardiogram and normal troponin levels. Atherosclerosis 2009; 206:251-257.

29 Gary T, Pichler M, Belaj K, Eller P, Hafner F, Gerger A, Brodman M: Lymphocyte-tomonocyte ratic a novel marker for critical limb ischernia in PAOD patients. Int J Clin Pract 2014;68:1483-1487.

30 Gidron Y, Armon T, Giluts H, Huleihel M: Psychological factors correlate meaningfully with percent-monocytes among acute coronary syndrome patients. Brain Behav Immun 2003;17:310-315.

31 Beck AT, Ward CH, Mendelson M, Mock J. Erbaugh J: An inventory for measuring depression. Arch Gen Psychiatry 1961;4:561571.

32 Ágoston G, Srili L Diagnostic questionnaires and symptom-rating scales in Füredi J, Tariska P (eds): The Hungarian Handbook of Psychiatry. Budapest, Medicina, 2001.

33 Rahe HR, Tolles RL: The brief stress and coping inventory: a useful stress management instrument. Int J Stress Manag 2002;9:61-70.

34 Rózsa S, K. N N, Csoboth Cs, Purebl Gy. Beōthy-Molnir A, Szebik I, Berghammer R, Réthelyi J. Skrabski Á, Kopp M: Stress and coping. Hungarian experiences with the Rahe Brief Stress and Coping Inventory (article in Hungarian). Mentailhigiené és Parichoszoma . tika 2005:6:275-294.

35 Cohen J: Statistical Power for the Behavioral Sciences, ed 3. New York, Acadernic Press, 1988.
36 Perneger TV: What's wrong with Bonferroni adjustments? BMT 1998;316:1236-1238.

37 Feise $\mathrm{RJ}$ : Do multiple outcome measures require p value adjustment? BMC Med Res Methodol 2002;2:8.

38 Glinicki P, Jeske W: Chromogranin A (CgA) - the influence of various factors in vivo and in vitro, and existing disorders on its concentration in blood. Endokrynol Pol 2010,61: 384-387.

39 Jansson AM, Resjo H, Omland T, Karlsson T, Hartford M, Plyvijierg A, Caidahl K- Prognostic value of circulating chromogranin $A$ levels in acute coronary syndromes. Eur Heart J $2009 ; 30-25-32$.

40 Clinicki P, Jeske $W$, Kapuscińska $R$, $Z_{g}$ liczyriski W: Comparison of chromogranin A (CgA) levels in serum and plasma (EDT $A_{2} \mathrm{~K}$ ) and the respective reference ranges in healthy males. Endokrynal Pol 2015;66: 53-56.

41 Cryer PE, Wortaman J, Shah SD, Nowak RM, Deftos L.J: Plasma chroenogranin A as a marker of sympathocromaffin activity in humans. Am J physiol Endocrinal Metab 1991; 260:E243-E246.

42 Ormland T, Dickstein K, Syversen U: Association between plasma chromogranin A concentration and long-term mortality after myocardial infarction. Am J Med 2003;114: $25-30$.

43 Duth P, Courties G, Wei Y, Leuschner F, Garbatov R, Robbins CS, Iwamoto Y, Thompson B, Carlson AL, Heidt T, Majmudar MD, Lasitschka P, Ftarodt M, Waterman P, Waring MT, Chicoine AT, van der Laan AM, Niessen HWM, Piek J., Rubin BB, Butany Stone JR, Katus HA, Murphy SA, Morrow DA, Sabatine MS, Vinegoni C, Moskowitz MA, Pittet MI, Libby P, Lin CP, Swirski FK, Weissleder R, Nahrendorf M: Myocardial infarction accelerates atherosclerosis. Nature 2012;487:325-329.

44 Tabas I: Rad matters made worse. Nature 2012;487:306-308.

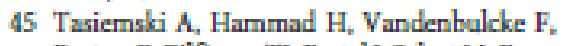
Breton C, Bilfinger TJ, Pestel], Saluet M: Presence of chromogranin-derived antimicrobial peptides in plasma during coronary artery bypass sargery and evidence of an immune origin of these peptides. Blood 2002;100-553559.
46 Black PH, Garbutt LD: Stress, inflammation and cardiovascular disease (review). J Psychosom Res 2002;52:1-23.

47 Kronfol Z, Remick DG: Cytokines and the brain: implications for clinical psychiatry. Am J Psychiatry 2000; 157:683-694.

48 Miller AH, Haroon E, Raison CL., Felger JC: Cytakine targets in the brain: impact on neu. rotransmitters and neurocircuits. Depress Anxiety 2013;30:297-306.

49 Nikkheslat N, Zunszain PA, Horowitz MA, Barbosa IG, Parker JA, Myint A-M, Schwarz M], Tylee AT, Carvalho LA, Pariante CM: In sufficient glucocorticoid signaling and elevated inflammation in coronary heart disease patients with comorbid depression. Brain Behav Immun 2015;48:8-18.

50 Miller GE, Chen E, Zhou ES: If it goes up, must it go down? Chronic stress and the hy. pothalamic-pituitary-adrenocortical axis in humans. Psychol Ball 2007;133-25-45.

51 Vandamme D, Landuyt B, Luyten W, Schoofs L. A comprehensive summary of L.-37, the factotum human cathelicidin peptide. Cell Immunol 2012;280-22-35.

52 Razanski $A$, Blumenthal $J A$, Davidson, $K$, Saab, PG, Kubransky L: The epidemiology. pathophysiology, and management of psy. chosocial risk factors in cardiac patients. J Am Coll Cardiol 2005; 45:637-651.

53 Friedler B, Craperer J, McCullough L: One is the deadliest number: the detrimental effects of social isolation on cerebrovascular diseases and cognition. Acta Neuropathol 2015;129: 493-509.

54 PirziC, Manzoli L., Mancini S, Bedetti G, Fontana F, Costa GM: Autonoenic nervous systern, inflammation and preclinical carotid atherosclerosis in depressed subjects with coronary risk factors Atherosclerosis 2010 ; 212-292-298.

55 Zeng W, StasonWB, Fournier S, Razavi M Ritter G, Strickler GK, Bhalotra SM, Shepard DS: Benefits and costs of intensive lifestyle modification programs for symptomatic coronary disease in MediCare beneficiaries. Am Heart J 2013;165:785-792.

\section{(C) Free Author Copy - for per-
sonal use only \\ ANY DISTRIBUTION OF THIS ARTILE WITHOUT WRITTEN CONSENT FROM S. KARGER AG, BASEL IS A VIOLATION OF THE COPYRIGHT. Witten permission to distrib- ute the PDF will be grealed equirst poyment of a per mission bee, which is tased on the number of excesses required. Please conlod permissionekurger.com}

